\title{
Quantum quench and thermalization to GGE in arbitrary dimensions and the odd-even effect
}

\author{
Parijat Banerjee, ${ }^{b, 1}$ Adwait Gaikwad, ${ }^{a}$ Anurag Kaushal ${ }^{a}$ and Gautam Mandal ${ }^{a}$ \\ ${ }^{a}$ Tata Institute of Fundamental Research, \\ Mumbai 400005, India \\ ${ }^{b}$ Johns Hopkins University, \\ 3400 N. Charles Street, Baltimore, MD 21218, U.S.A. \\ E-mail: pbanerjee5@students.iiserpune.ac.in, \\ adwait@theory.tifr.res.in, anurag.kaushal@theory.tifr.res.in, \\ mandal@theory.tifr.res.in
}

ABSTRACT: In many quantum quench experiments involving cold atom systems the postquench phase can be described by a quantum field theory of free scalars or fermions, typically in a box or in an external potential. We will study mass quench of free scalars in arbitrary spatial dimensions $d$ with particular emphasis on the rate of relaxation to equilibrium. Local correlators expectedly equilibrate to GGE; for quench to zero mass, interestingly the rate of approach to equilibrium is exponential or power law depending on whether $d$ is odd or even respectively. For quench to non-zero mass, the correlators relax to equilibrium by a cosine-modulated power law, for all spatial dimensions $d$, even or odd. We briefly discuss generalization to $O(N)$ models.

Keywords: Field Theories in Higher Dimensions, Field Theories in Lower Dimensions, Integrable Field Theories

ARXIV EPrint: 1910.02404

\footnotetext{
${ }^{1}$ Work started during a research project in TIFR.
} 


\section{Contents}

1 Introduction and summary 1

2 Quantum quench in free scalar theories: review 4

2.1 Quantum quench from the ground state 5

$\begin{array}{lll}2.1 .1 & \text { Two-point functions } & 7\end{array}$

2.2 More general quantum quench: from squeezed states 9

$\begin{array}{ll}\text { 2.2.1 The squeezed state 2-point function } & 10\end{array}$

$\begin{array}{lll}2.3 & \text { gCC correlators encompass all cases } & 11\end{array}$

3 Time-dependence of two-point functions 11

$\begin{array}{lll}3.1 \text { General remarks } & 12\end{array}$

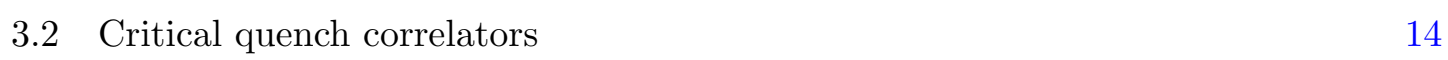

3.2.1 Time-dependent part of the $\langle\phi \phi\rangle$ correlator $\quad 14$

$\begin{array}{lll}3.2 .2 & \text { Large time behaviour for odd } d & 14\end{array}$

$\begin{array}{lll}3.2 .3 & \text { Large time behaviour for even } d & 16\end{array}$

$\begin{array}{ll}3.2 .4 \partial_{i} \phi \partial_{i} \phi \text { and } \partial_{t} \phi \partial_{t} \phi \text { correlator } & 19\end{array}$

$\begin{array}{ll}3.3 \text { Massive quench correlators } & 19\end{array}$

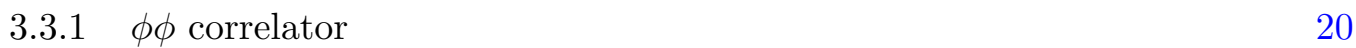

3.3.2 $\partial_{t} \phi \partial_{t} \phi$ and $\partial_{i} \phi \partial_{i} \phi$ correlators 22

3.4 Some comments on 2-point functions in critical vs. massive quench 23

4 The generalized Gibbs ensemble (GGE) 24

4.1 GGE 24

$\begin{array}{lll}4.2 & \text { Equilibration to GGE } & 25\end{array}$

5 Geometrical interpretation of the correlators in the CC state $\quad \mathbf{2 6}$

5.1 Comments on approach to thermalization and the odd-even effect 27

$\begin{array}{ll}5.2 & \text { The thermal auto-correlator } \\ \end{array}$

$6 \quad$ Kaluza-Klein interpretation of thermal correlators 31

$\begin{array}{lll}7 & \text { Discussion } & 32\end{array}$

A Dirichlet boundary state and relation to post-quench state 34

$\begin{array}{ll}\text { B Recursion relation } & 35\end{array}$

C Details of critical quench calculations $\quad 36$

$\begin{array}{lll}\text { C.1 } 2+1 \text { dimensions } & 36\end{array}$

$\begin{array}{lll}\text { C.1.1 CC state } & 36\end{array}$

C.1.2 Ground state 36

$\begin{array}{lll}\text { C.2 } & 3+1 \text { dimensions } & 37\end{array}$ 
$\begin{array}{lll}\text { C.2.1 CC correlator } & 37\end{array}$

$\begin{array}{lll}\text { C.2.2 } & \mathrm{gCC}_{4} \text { correlator } & 39\end{array}$

$\begin{array}{ll}\text { C.2.3 Ground state correlator } & 40\end{array}$

C.2.4 The thermal correlator 41

C.3 $4+1$ dimensions 42

$\begin{array}{lll}\text { C.3.1 CC correlator } & 42\end{array}$

C.3.2 Ground state correlator 43

$\begin{array}{lll}\text { C.3.3 The thermal correlator } & 43\end{array}$

D GGE correlator $\quad 44$

\section{Introduction and summary}

Thermalization in integrable systems has been an important area of study for more than a decade [1-17]. We should clarify, at the outset, what is meant here by thermalization. For systems with an infinite number of local conserved charges $Q_{i}, i=1,2, \ldots,{ }^{1}$ an appropriate description of the equilibrium ensemble is provided by the generalized Gibbs ensemble (GGE), described by a density matrix

$$
\rho_{\mathrm{GGE}}\left(\mu_{i}\right)=\frac{1}{Z} \exp \left[-\sum_{i} \mu_{i} Q_{i}\right], \quad Z=\operatorname{tr} \exp \left[-\sum_{i} \mu_{i} Q_{i}\right] .
$$

Here $\mu_{i}$ 's denote an infinite number of chemical potentials conjugate to the conserved charges. The question of interest in the above studies is, whether local correlators in a time-dependent state relax, in the infinite time limit, to their values in a GGE. We will call this "thermalization" in a generalized sense. More specifically, such a thermalization should be called "local thermalization" or "subsystem thermalization" since we focus on relaxation of local correlators confined to a finite subpart of the system with respect to which the rest of the system can be regarded as a bath.

It is clearly important to figure out which class of initial states, for a given system, leads to local thermalization in the sense defined above. In general, this is not an easy question to answer; examples of initial states abound, which do not thermalize. The classic 1955 theoretical simulation of Fermi-Pasta-Ulam-Tsingou [18] and the relatively recent quantum Newton's cradle experiment by Kinoshita et al. [19] show examples of these in one dimensional systems.

A subject of extensive theoretical and experimental investigation has been that of initial states generated by quantum quenches, especially in one dimensional systems (see the references listed in the beginning). A question of clear importance, with regard to local thermalization of integrable systems, is the following. Consider a quantum quench in which the post-quench Hamiltonian is integrable. Construct the reduced density matrix (RDM)

\footnotetext{
1 "Local" charges imply $Q_{i}=\int d^{d} x \mathcal{Q}_{i}(x)$ in the continuum language.
} 
$\rho_{A}(t)$, of a finite subregion $A$ of the system. ${ }^{2}$ The RDM is defined as $\rho_{A}(t)=\operatorname{tr}_{A^{c}} \rho(t)$, $\rho(t)=|\psi(t)\rangle\langle\psi(t)|$, where the partial trace is done over the degrees of freedom localized in the complementary region $A^{c}$. Let us also define the RDM of a GGE as $\rho_{A}\left(\mu_{i}\right)=$ $\operatorname{tr}_{A^{c}} \rho_{\mathrm{GGE}}\left(\mu_{i}\right)$. Local thermalization to GGE would require that $\rho_{A}(t) \rightarrow \rho_{A}\left(\mu_{i}\right)$ at long times. ${ }^{3}$ For thermalization to ordinary Gibbs ensemble in $1+1$ dimensional conformal field theories, this result, along with the relaxation rate, was predicted in e.g. [20], where the initial state was the Calabrese-Cardy (CC) state [21-23] (we will say more on these below). A similar question for integrable models was addressed by MSS [24], and independently by Cardy [25], where a more general class of initial states (called generalized CalabreseCardy (gCC) states by MSS) was shown to locally thermalize to GGE in the sense described above. ${ }^{4}$ The relaxation rate was computed, and in MSS, compared with quasinormal decays in a gravitational dual, a higher spin black hole, for suitable conformal field theories.

The gCC ansatz for post-quench states was a natural generalization of the CC ansatz to integrable models. In particular, in case of free scalar field theories, ${ }^{5}$ for a sudden quench of mass to zero, the post-quench state was indeed shown to be of the gCC form in [34] (see also [3]). Exact evaluation of time-dependent correlators in such states was performed in MPS [35], without recourse to perturbative resummation as in MSS [24]; the holographic comparison to black holes was also shown to be exact. Several aspects of these considerations, including the appearance of states of the gCC form, were discussed in the context of interacting integrable models in [34, 36-39] (for a picture of interacting integrable models in terms of quasiparticles with nontrivial scattering, see [40]).

The results detailed above are in the context of $1+1$ dimensional quantum field theories. Generalization to higher dimensions, though not equally extensively, has been discussed in higher dimensions (see, e.g. [3] for free scalar fields, and [41-43] for $O(N)$ models with interactions). In the present paper we study mass quench in $d+1$ dimensional free scalar field theories and analyze local thermalization of correlators. The pre-quench state is allowed to be an arbitrary squeezed state of the pre-quench modes; choosing this state appropriately, we show that, the post-quench state can be tailored to be completely general gCC state. We explicitly find that expectation values and local correlators in the most general $\mathrm{gCC}$ state evolve at long times to their respective values in an appropriate GGE. The main result we find is that, for a quench to zero mass, correlators relax to their GGE values as an exponential $\left(\sim e^{-\gamma t}\right)$ or as a power law $\sim 1 / t^{p}$, depending on whether the number of space dimensions is odd or even, respectively; we call this result the odd-even effect. We compute the relaxation for quench to nonzero mass as well, where the correlators have a cosine-modulated power law relaxation in any dimension. We also briefly discuss generalization of our results to an $O(N)$ model. As mentioned above, some

\footnotetext{
${ }^{2}$ The size of the full system will be assumed much larger than any other length scale in this paper.

${ }^{3}$ Here the chemical potentials $\mu_{i}$ are to be determined by matching the conserved charges between the GGE and the pure state under consideration.

${ }^{4}$ The issue of thermalization to GGE from an even more general class of states, with nontrivial cumulants, has also been discussed; see, e.g. [26, 27].

${ }^{5}$ Quantum quench in free fermion theories, or hard core bosons, has been discussed in [28-32]. Universal short time behaviour of quantum quenches in free field theories, including Kibble-Zurek quenches, has been discussed in [31, 33].
} 
Long time behaviour of equal-time two-point functions $G(t)$ (note the odd-even effect)

\begin{tabular}{|c|c|c|}
\hline & $m_{\text {out }}=0$ & $m_{\text {out }} \neq 0 \ll m_{\text {in }}$ \\
\hline$d=1$ & $e^{-\gamma t}$ & $\frac{\cos \left(2 m_{\text {out }} t+\delta\right)}{t^{1 / 2}}$ \\
\hline$d=2$ & $\frac{1}{t^{2}}$ & $\frac{\cos \left(2 m_{\text {out }} t+\delta\right)}{t}$ \\
\hline odd $d>1$ & $e^{-\gamma t}$ & $\frac{\cos \left(2 m_{\text {out }} t+\delta\right)}{t^{d / 2}}$ \\
\hline even $d>2$ & $\frac{1}{t^{d-2}}$ &, \\
\hline
\end{tabular}

Table 1. Here $d$ refers to the number of spatial dimensions. The table displays the structure of an equal time two-point function $G(t)$. For $d>2, G(t) \equiv\langle\phi(x, t) \phi(y, t)\rangle$; for $d=1,2, m_{\text {out }}=0$ this quantity diverges at long times, hence we display $G(t)=\left\langle\partial_{t} \phi(x, t) \partial_{t} \phi(y, t)\right\rangle$ (in all cases, we drop the spatial dependence). The decay coefficients $\gamma$ are given in the text (see e.g. table 4 which lists various values of $\gamma$ for $d=3$ : these values depend on the specific initial state); the exponential decay has a power law prefactor in case of ground state quench. The phase factors $\delta$ are given by $d \pi / 4$. Note that the power laws in the massless case (for even dimensions) differ from those in the massive case.

of our methods have overlaps with earlier works, which we will indicate as we go along. The paper is nevertheless presented, even at the cost of some repetition, in a detailed selfcontained way, using continuum quantum field theory formalism. This is especially done for convenience of readers outside of condensed matter-statistical physics community.

We summarize the main points in table 1.

The paper is organized as follows.

In section 2 we discuss the main formalism of how to describe the post-quench state as a Bogoliubov transform of the out-vacuum, leading to its identification (with the help of appendix A) as a generalized Calabrese-Cardy state (2.12). We discuss starting with ground states as well as squeezed states in the massive phase. Section 2.2.1 gives the formulae for the time-dependent two-point functions.

In section 3 we explicitly calculate the time-dependent part of the two-point function (additional material is provided in appendices $\mathrm{B}$ and $\mathrm{C}$ ). Tables 4,5 and 6 show the decay of the time-dependent part for critical quench $\left(m_{\text {out }}=0\right)$ for the number of spatial dimensions $d=1,2,3,4$; the results for general dimensions $d$ are obtained by using a recursion relation. For non-critical quench $\left(m_{\text {out }} \neq 0\right)$, tables 7,8 and 9 present the time-dependent part of the two-point functions for $d=1,2,3,4$; the formula for the general dimension is given in (3.27). The overall behaviour is as indicated in table 1 above. The relaxation to equilibrium for critical quench shows an odd-even effect, as explained above, namely exponential/power-law decay to equilibrium in odd/even number of space dimensions, respectively.

The fact that the time-dependent parts of the two-point functions vanish at large times, already implies that these correlators (and hence all correlators, by the application of Wick's theorem), asymptotically equilibrate. In section 4, using results from appendix D, we explicitly show that the time-independent parts of the two-point functions, to which they equilibrate, are given by two-point functions in a generalized Gibbs ensemble(GGE). This constitutes a proof of thermalization of arbitrary local correlators (section 4.2). (This 


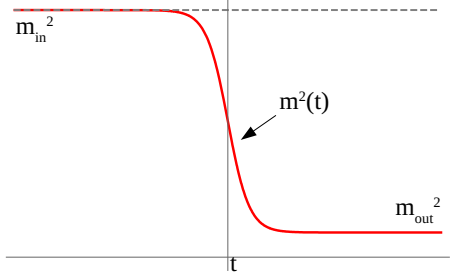

(a) Mass quench

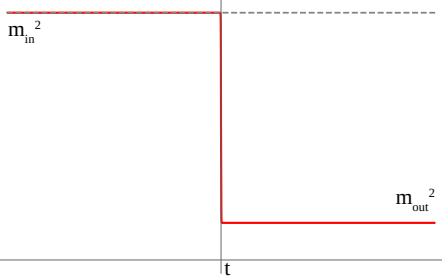

(b) Quench in sudden limit

Figure 1. Quantum quench of mass of a scalar field in $d+1$ dimensions with the protocol in eq. (2.2). This is shown in the left panel. For simplicity, we will consider a sudden quench for most of the paper, in which case, the mass profile looks like the figure on the right.

is analogous to a similar result in [3], section VI.B; our result constitutes a simple generalization of this to $d+1$ dimensions.)

In section 5, building on the formalism of [41], we look at the geometrical interpretation of two-point functions in the CC state, which allows a better understanding of the odd-even effect.

In section 6 we calculate the thermal correlator for purely spatial separation and make a few remarks about its interpretation in terms of a Kaluza-Klein reduction along the thermal circle. For high enough temperature, or equivalently large enough spatial separation, only the Kaluza-Klein zero mode contribution survives, which has a power law behaviour. This result holds in any dimension, even or odd.

In section 7 we conclude with some comments with a brief discussion of ongoing work on $O(N)$ models.

\section{Quantum quench in free scalar theories: review}

In this section, we review mass quench in free scalar field theories in $d+1$ dimensions. Most of the formalism described here are available from, or can be easily obtained from, the existing literature cited in the Introduction section. The main purpose of this section is to set up the notation and make the paper self-contained.

The basic set-up for mass quench is as follows. Consider a relativistic scalar free field theory in $d$ spatial dimensions, with a time dependent mass (see figure 1)

$$
\mathcal{L}=\frac{1}{2}\left(\partial_{t} \phi \partial_{t} \phi-\partial_{i} \phi \partial_{i} \phi-m^{2}(t) \phi \phi\right) ; \quad i=1, \cdots, d
$$

In Fourier space,

$$
\phi(\vec{x}, t)=\int \frac{d^{d} k}{(2 \pi)^{d}} e^{i \vec{k} \cdot \vec{x}} \phi(\vec{k}, t)
$$

the equation of motion for the Fourier mode $\phi(\vec{k}, t)$ (similar equation for $\phi^{*}(\vec{k}, t)$ ) is

$$
-\partial_{t}^{2} \phi(\vec{k}, t)-m^{2}(t) \phi(\vec{k}, t)=|\vec{k}|^{2} \phi(\vec{k}, t)
$$

For every $\vec{k}$, this can be identified with a Schrodinger problem on a line (coordinatized by $y$, say), with the identifications

$$
t \rightarrow y, m^{2}(t) \rightarrow-V(y),|\vec{k}|^{2} \rightarrow E, \phi(\vec{k}, t) \rightarrow \psi_{E}(y)
$$


In the Schrodinger problem there are two equivalent bases of solutions: one which corresponds to particles coming in from the left: $\psi_{E}(y)=$ linear combination of $u_{\text {in }}(E, y)$, $u_{\text {in }}^{*}(E, y)$, where $u_{\text {in }}(E, y) \sim e^{i \omega_{\text {in }} y}$ as $y \rightarrow-\infty$, and another which corresponds to particles coming in from the right, $u_{\text {out }}(y) \sim e^{-i \omega_{\text {out }} y}$ as $y \rightarrow+\infty$. Taking cue from this, we have two sets of normal mode expansions:

$$
\begin{aligned}
\phi(\vec{k}, t) & =a_{\text {in }}(\vec{k}, t) u_{\text {in }}(\vec{k}, t)+c c, u_{\text {in }} \sim e^{-i \omega_{\text {in }} t}, t \rightarrow-\infty, \\
\phi(\vec{k}, t) & =a_{\text {out }}(\vec{k}, t) u_{\text {out }}(\vec{k}, t)+c c, u_{\text {out }} \sim e^{-i \omega_{\text {out }} t}, t \rightarrow \infty \\
\omega_{\text {in }} & =\sqrt{|\vec{k}|^{2}+m_{\text {in }}^{2}}, \quad \omega_{\text {out }}=\sqrt{|\vec{k}|^{2}+m_{\text {out }}^{2}}
\end{aligned}
$$

Here. The two basis sets are of course linear combinations of each other

$$
u_{\text {in }}(\vec{k}, t)=\alpha(\vec{k}) u_{\text {out }}(\vec{k}, t)+\beta(\vec{k}) u_{\text {out }}^{*}(-\vec{k}, t)
$$

which implies

$$
\begin{aligned}
a_{\text {in }} & =\alpha^{*}(\vec{k}) a_{\text {out }}(\vec{k})-\beta^{*}(\vec{k}) a_{\text {out }}^{\dagger}(-\vec{k}) \\
a_{\text {out }} & =\alpha(\vec{k}) a_{\text {in }}(\vec{k})+\beta^{*}(\vec{k}) a_{\text {in }}^{\dagger}(-\vec{k})
\end{aligned}
$$

Here $\alpha, \beta$ are the Bogoliubov coefficients. We take the mass profile to be (see figure 1a)

$$
m^{2}(t)=\frac{1}{2}\left(m_{\text {in }}^{2}+m_{\text {out }}^{2}\right)-\frac{1}{2}\left(m_{\text {in }}^{2}-m_{\text {out }}^{2}\right) \tanh (\rho t)
$$

Further we do all our calculations in the sudden limit $\rho \rightarrow \infty,{ }^{6}$ (see figure 1b). For the quench protocol eq. (2.2), Bogoliubov coefficients are easy to compute explicitly [44]; in the sudden limit (more details can be found in the $1+1$ dimensional context in [35])

$$
\alpha(k)=\frac{1}{2} \frac{\omega_{\text {out }}+\omega_{\text {in }}}{\sqrt{\omega_{\text {out }} \omega_{\text {in }}}}, \beta(k)=\frac{1}{2} \frac{\omega_{\text {out }}-\omega_{\text {in }}}{\sqrt{\omega_{\text {out }} \omega_{\text {in }}}}
$$

In this limit the in- and out- waves also become especially simple

$$
u_{\text {in }}(k, t)=\frac{e^{-i \omega_{\text {in }} t}}{\sqrt{2 \omega_{\text {in }}}}, u_{\text {out }}(k, t)=\frac{e^{-i \omega_{\text {out }} t}}{\sqrt{2 \omega_{\text {out }}}}
$$

The final mass is arbitrary but taken to be less than the initial mass i.e. $m_{\text {out }}<m_{\text {in }}$. We also study the theory when the final mass is taken to be zero, when the final theory is critical. As we will see the more interesting results are obtained in the critical quench.

\subsection{Quantum quench from the ground state}

A natural pre-quench initial state (just before $t=0$ ) is the ground state $\left|0_{\text {in }}\right\rangle$ of the initial Hamiltonian $H_{\text {in }}$, which is also the zero-particle state defined by the oscillators $a_{\text {in }}|0\rangle_{\text {in }}=0$. Just after $t=0$, the state remains $|\psi(0)\rangle=|0\rangle_{\text {in }}$ (remember we are working in the sudden quench limit, therefore the state has no time to change). We then define $|\psi(t)\rangle=e^{-i H t}|\psi(0)\rangle$ where $H:=H_{\text {out }}$ is the final Hamiltonian.

\footnotetext{
${ }^{6}$ This limit has to be understood in the sense explained in detail in [35].
} 
The 'in' ground state can be written in terms of the out ground state through a Bogoliubov transformation ${ }^{7}$

$$
|\psi(0)\rangle=\left|0_{\text {in }}\right\rangle=\exp \left[\frac{1}{2} \sum_{\vec{k}} \gamma(k) a_{\text {out }}^{\dagger}(\vec{k}) a_{\text {out }}^{\dagger}(-\vec{k})\right]\left|0_{\text {out }}\right\rangle
$$

where $\gamma(k)=\beta^{*}(k) / \alpha^{*}(k)$ depends on the Bogoliubov coefficients and is only a function of $k:=|\vec{k}| .^{8}$ Further the 'in' ground state is also related to the Dirichlet boundary state [24] (see appendix A for more details)

$$
|D\rangle=\exp \left[-\frac{1}{2} \sum_{\vec{k}} a_{\text {out }}^{\dagger}(\vec{k}) a_{\text {out }}^{\dagger}(-\vec{k})\right]\left|0_{\text {out }}\right\rangle
$$

through the relation

$$
\left|0_{\text {in }}\right\rangle=\exp \left[\frac{1}{2} \sum_{\vec{k}} \kappa(k) a_{\text {out }}^{\dagger}(\vec{k}) a_{\text {out }}(\vec{k})\right]|D\rangle
$$

where

$$
\kappa(k)=-\frac{1}{2} \log (-\gamma(k))
$$

In general one can expand $\kappa(k)$ for small $k$ :

$$
\kappa(k)=\sum_{i=1}^{\infty} \kappa_{i} k^{i-1}=\kappa_{1}+\kappa_{2} k+\kappa_{3} k^{2}+\ldots
$$

With this, the expression for $\left|0_{\text {in }}\right\rangle$ becomes

$$
|\psi(0)\rangle=\left|0_{\text {in }}\right\rangle=\exp \left[-\sum_{i=1}^{\infty} \kappa_{i} Q_{i}\right]|D\rangle
$$

where

$$
Q_{i}=\sum_{\vec{k}}|\vec{k}|^{i-1} a_{\text {out }}^{\dagger}(\vec{k}) a_{\text {out }}(\vec{k})
$$

are consereved charges (they obviously all commute with the 'out' Hamiltonian $H=\sum_{\vec{k}}$ $\left.\sqrt{|\vec{k}|^{2}+m_{\text {out }}^{2}} a_{\text {out }}^{\dagger}(\vec{k}) a_{\text {out }}(\vec{k})\right) .{ }^{9}$ It is easy to explicitly compute the $\kappa_{i}$ coefficients by using the definition of $\kappa(k)$ in terms of the Bogoliubov coefficients which are given in (2.3):

$$
\kappa(k)=\frac{1}{2} \log \left(\frac{\sqrt{k^{2}+m^{2}}+\sqrt{k^{2}+m_{\text {out }}^{2}}}{\sqrt{k^{2}+m^{2}}-\sqrt{k^{2}+m_{\text {out }}^{2}}}\right)
$$

\footnotetext{
${ }^{7}$ This is easily checked by noting that the right hand side is annihilated by $a_{\text {in }}=\alpha^{*}(\vec{k}) a_{\text {out }}(\vec{k})-$ $\beta^{*}(\vec{k}) a_{\text {out }}^{\dagger}(-\vec{k})$.

${ }^{8}$ Note that, because we focus on homogeneous quench protocols in this paper, we have rotational invariance in the $d$ spatial dimensions. Hence the Bogoliubov coefficients are a function of $k=|\vec{k}|$.

${ }^{9}$ For free scalars, the number operators $N(k)=a_{\text {out }}^{\dagger}(\vec{k}) a_{\text {out }}(\vec{k})$ are themselves conserved, and provide an alternative basis of the algebra of conserved charges.
} 
Note that henceforth we will call $m_{\text {in }}=: m$ for simplicity. It is assumed that $m>m_{\text {out }}$. From the above equation, the small $k$ expansion can be easily found.

For $m_{\text {out }}=0$, the expansion contains only odd powers of $k$, thus the even $\kappa_{i}$ 's are non-zero, e.g.

$$
\kappa_{2}=\frac{1}{m}, \kappa_{4}=-\frac{1}{6 m^{3}}, \ldots
$$

By contrast, for $m_{\text {out }} \neq 0$, only even powers of $\mathrm{k}$ survive, leading to odd $\kappa_{i}$ 's, e.g.

$$
\kappa_{1}=\frac{1}{2} \log \frac{m+m_{\text {out }}}{m-m_{\text {out }}}, \kappa_{3}=\frac{1}{2 m m_{\text {out }}}, \ldots
$$

Relation to a generalized Calabrese-Cardy (gCC) state. In case of critical quench $\left(m_{\text {out }}=0\right)$, the post-quench dynamics is conformal. For critical quenches leading to a generic (non-integrable) conformal field theory, Calabrese and Cardy [3, 21, 23] postulated the following form for the post-quench wavefunction ${ }^{10}$

$$
|C C\rangle=\exp [-\kappa H]|B d\rangle
$$

where, $\kappa$ is given by the inverse of the mass gap characterizing the initial state (before the quench). The state $|B d\rangle$ is a "boundary state" representing a conformal state subject to an appropriate boundary condition at imaginary time $t=-i \kappa$.

In case the post-quench conformal theory is integrable, characterized by an infinite number of charges $Q_{i}$, a more appropriate ansatz for the post-quench state is the gCC (generalized Cardy-Calabrese) state (see [3], we are borrowing the name from [24] where quantum quench of gCC states was studied in detail in $1+1$ dimensions):

$$
|g C C\rangle=\exp \left[-\sum_{i=1}^{\infty} \kappa_{i} Q_{i}\right]|B d\rangle
$$

The state (2.6) is clearly a special case of such a state, (see [35] for more details in the $1+1$ dimensional free field theories). Note that the Dirichlet state $|D\rangle$ is a particular example of a boundary state. As mentioned above (2.9), here only the even charges $Q_{2 n}, n>0$ are non-zero; note that $Q_{2}=H$, as can be seen from the definition (2.7).

In case of a massive quench, the state (2.6) is of the form (2.12), where the conformal boundary state $|B d\rangle$ is replaced by the Dirichlet boundary state $|D\rangle$. Although the postquench theory is not conformal in this case, we will continue to use the name gCC for the state (2.6). In fact we will continue to use this nomenclature also for states like (2.20) obtained by noncritical quench from squeezed states.

\subsubsection{Two-point functions}

We will be interested in computing quantities like

$$
\left\langle\psi\left(t_{1}\right)\left|O\left(x_{1}\right) O\left(x_{2}\right)\right| \psi\left(t_{2}\right)\right\rangle=\left\langle\psi(0)\left|O\left(\overrightarrow{x_{1}}, t_{1}\right) O\left(\overrightarrow{x_{2}}, t_{2}\right)\right| \psi(0)\right\rangle
$$

\footnotetext{
${ }^{10}$ One assumes here that the quench takes a finite time to end, say from $t=0$ to $t=t_{0}$. In this paper, we are interested in a sudden quench which takes place at $t=0$, i.e. $t_{0}=0$.
} 
The operators appearing on the r.h.s. $O(x, t)=e^{i H_{\text {out }} t} O(x) e^{-i H_{\text {out }} t}$ are in the Heisenberg picture defined with the Hamiltonian $H_{\text {out }}$. This is applicable for $t \geq 0$. For time evolution to $t<0$ we must use the Hamiltonian $H_{\mathrm{in}}$. With this understanding it is clear that the above definition comes with the prescription $t_{1}, t_{2} \geq 0$. The relation to time-ordered correlator is noted below (2.16). This definition includes in particular, the equal-time correlator (ETC)

$$
\left\langle 0_{\text {in }}\left|O\left(\overrightarrow{x_{1}}, t\right) O\left(\overrightarrow{x_{2}}, t\right)\right| 0_{\text {in }}\right\rangle
$$

In our theory these are related to the ground-state two-point function

$$
\begin{aligned}
& G\left(\overrightarrow{x_{1}}, t_{1} ; \overrightarrow{x_{2}}, t_{2}\right) \equiv\left\langle 0_{\text {in }}\left|\phi\left(\overrightarrow{x_{1}}, t_{1}\right) \phi\left(\overrightarrow{x_{2}}, t_{2}\right)\right| 0_{\text {in }}\right\rangle=\int \frac{d^{d} k}{(2 \pi)^{d}} u_{\text {in }}\left(\vec{k}, t_{1}\right) u_{\text {in }}^{*}\left(\vec{k}, t_{2}\right) e^{i \vec{k} \cdot\left(\overrightarrow{x_{1}}-\overrightarrow{x_{2}}\right)} \\
& =\int \frac{d^{d} k}{(2 \pi)^{k}}\left[|\alpha(k)|^{2} u_{\text {out }}\left(k, t_{1}\right) u_{\text {out }}^{*}\left(k, t_{2}\right)+\alpha(k) \beta^{*}(k) u_{\text {out }}\left(k, t_{1}\right) u_{\text {out }}\left(-k, t_{2}\right)\right. \\
& \left.\quad+\alpha^{*}(k) \beta(k) u_{\text {out }}^{*}\left(-k, t_{1}\right) u_{\text {out }}^{*}\left(k, t_{2}\right)+|\beta(k)|^{2} u_{\text {out }}^{*}\left(-k, t_{1}\right) u_{\text {out }}\left(-k, t_{2}\right)\right] e^{i k \cdot\left(x_{1}-x_{2}\right)}
\end{aligned}
$$

Using the expressions (2.3) and (2.4), the ground-state two-point function becomes

$$
\begin{aligned}
& \left\langle 0_{\text {in }}\left|\phi\left(\overrightarrow{x_{1}}, t_{1}\right) \phi\left(\overrightarrow{x_{2}}, t_{2}\right)\right| 0_{\text {in }}\right\rangle \\
& =\int \frac{d^{d} k}{(2 \pi)^{d}} \frac{e^{i \vec{k} \cdot\left(\overrightarrow{x_{1}}-\overrightarrow{x_{2}}\right)}}{4 \sqrt{\left(k^{2}+m^{2}\right)}\left(k^{2}+m_{\text {out }}^{2}\right)}\left[\left(2 k^{2}+m^{2}+m_{\text {out }}^{2}\right) \cos \left(\sqrt{k^{2}+m_{\text {out }}^{2}}\left(t_{1}-t_{2}\right)\right)\right. \\
& \left.+\left(m_{\text {out }}^{2}-m^{2}\right) \cos \left(\sqrt{k^{2}+m_{\text {out }}^{2}}\left(t_{1}+t_{2}\right)\right)-2 i \sqrt{\left(k^{2}+m^{2}\right)\left(k^{2}+m_{\text {out }}^{2}\right)} \sin \left(\sqrt{k^{2}+m_{\text {out }}^{2}}\left(t_{1}-t_{2}\right)\right)\right]
\end{aligned}
$$

As mentioned above this correlator is not time-ordered. The time-ordered correlator can be obtained from this by replacing $t_{1}-t_{2}$ by $\left|t_{1}-t_{2}\right|$ but leaving $t_{1}+t_{2}$ unaltered. As a consequence, the ETC's considered here are already time-ordered. The expression above simplifies for $t_{1}=t_{2}$ (ETC):

$$
\begin{aligned}
\left\langle 0_{\text {in }}\left|\phi\left(\overrightarrow{x_{1}}, t\right) \phi\left(\overrightarrow{x_{2}}, t\right)\right| 0_{\text {in }}\right\rangle= & \int \frac{d^{d} k}{(2 \pi)^{d}} \frac{e^{i \vec{k} \cdot\left(\overrightarrow{x_{1}}-\overrightarrow{x_{2}}\right)}}{4 \sqrt{k^{2}+m^{2}}\left(k^{2}+m_{\text {out }}^{2}\right)} \\
& {\left[\left(m_{\text {out }}^{2}-m^{2}\right) \cos \left(2 t \sqrt{k^{2}+m_{\text {out }}^{2}}\right)+2 k^{2}+m^{2}+m_{\text {out }}^{2}\right] }
\end{aligned}
$$

We also look at the 2-point function of the $\partial_{t} \phi$ operator. Note that this correlator is directly obtainable from the unequal time two-point function $\langle\phi \phi\rangle$ (2.16) by applying the operator $\left.\partial_{t_{1}} \partial_{t_{2}}\right|_{t_{1}=t_{2}=t}$. Since the unequal time correlator is of the form $f\left(t_{1}-t_{2}\right)+g\left(t_{1}+t_{2}\right)$, it follows that $\left\langle 0_{\text {in }}\left|\partial_{t_{1}} \phi\left(\overrightarrow{x_{1}}, t_{1}\right) \partial_{t_{2}} \phi\left(\overrightarrow{x_{2}}, t_{2}\right)\right| 0_{\text {in }}\right\rangle$ is of the form $-f^{\prime \prime}\left(t_{1}-t_{2}\right)+g^{\prime \prime}\left(t_{1}+t_{2}\right)$. Hence the equal-time two-point function of $\partial_{t} \phi$ is of the form $-f^{\prime \prime}(0)+g^{\prime \prime}(2 t)$; in particular, the time-dependent part of this correlator is $\partial_{t}^{2}$ applied to the time-dependent part of (2.17). 
By explicit calculation, one finds the expression

$$
\begin{aligned}
\left\langle 0_{\text {in }}\left|\partial_{t} \phi\left(\overrightarrow{x_{1}}, t\right) \partial_{t} \phi\left(\overrightarrow{x_{2}}, t\right)\right| 0_{\text {in }}\right\rangle= & \int \frac{d^{d} k}{(2 \pi)^{d}} \frac{e^{i \vec{k} \cdot\left(\overrightarrow{x_{1}}-\overrightarrow{x_{2}}\right)}}{4 \sqrt{k^{2}+m^{2}}} \\
& {\left[-\left(m_{\text {out }}^{2}-m^{2}\right) \cos \left(2 t \sqrt{k^{2}+m_{\text {out }}^{2}}\right)+2 k^{2}+m^{2}+m_{\text {out }}^{2}\right] }
\end{aligned}
$$

which indeed follows the general form $-f^{\prime \prime}(0)+g^{\prime \prime}(2 t)$ mentioned above.

A primary motivation for considering two-point functions of the derivative operators $\left\langle\partial_{t} \phi \partial_{t} \phi\right\rangle$ is as follows. In low dimensions the time-dependent part $g(2 t)$ of the equal time correlator $\left\langle\partial_{t} \phi \partial_{t} \phi\right\rangle$ (2.17) grows in time which masks the transients that signal thermalization (it grows linearly in time in $d=1$, and logarithmically in $d=2$ ). The extra time derivatives, leading to $g^{\prime \prime}(2 t)$, get rid of these divergences.

For the same reason, we may also consider the correlators of the spatial derivatives $\vec{\partial} \phi$,

$$
\left\langle 0_{\text {in }}\left|\partial_{i} \phi\left(\vec{x}_{1}, t_{1}\right) \partial_{j} \phi\left(\vec{x}_{2}, t_{2}\right)\right| 0_{\text {in }}\right\rangle=\partial_{x_{1 i}} \partial_{x_{2 j}}\left\langle\phi\left(\vec{x}_{1}, t_{1}\right) \phi\left(\vec{x}_{2}, t_{2}\right)\right\rangle,
$$

The extra derivatives ensure decay with increasing $|\vec{x}|$ (in $d=1,2\langle\phi \phi\rangle$ grows, respectively, linearly and logarithmically with $|\vec{x}|)$. It is enough for this purpose to focus on

$$
\left\langle 0_{\text {in }}\left|\partial_{i} \phi\left(\vec{x}_{1}, t\right) \partial_{i} \phi\left(\vec{x}_{2}, t\right)\right| 0_{\text {in }}\right\rangle=-\partial_{i} \partial_{i}\left\langle\phi\left(\vec{x}, t_{1}\right) \phi\left(\overrightarrow{0}, t_{2}\right)\right\rangle, \quad \vec{x}=\vec{x}_{1}-\vec{x}_{2}
$$

We will discuss more details of two-point functions of these derivative operators and their relations in section 2.2 .1 below.

\subsection{More general quantum quench: from squeezed states}

As we saw above, the post quench state (2.6) is built out of infinite number of chemical potentials acting on the Dirichlet boundary state. However certain special gCC states can be obtained if the initial state are chosen to be specific squeezed states of the pre-quench Hamiltonian (for an early reference, see [3])

$$
|\psi(0)\rangle=|f\rangle_{\text {in }} \equiv \exp \left[\frac{1}{2} \sum_{k} f(\vec{k}) a_{\text {in }}^{\dagger}(\vec{k}) a_{\text {in }}^{\dagger}(-\vec{k})\right]\left|0_{\text {in }}\right\rangle
$$

This is just the Bogoliubov transformation of $\left|0_{\text {in }}\right\rangle$. As the $\left|0_{\text {in }}\right\rangle$ state can itself be written as a Bogoliubov transform of $\left|0_{\text {out }}\right\rangle$, the post quench state $\left|f_{\text {in }}\right\rangle$ is a composite Bogoliubov transform of $\left|0_{\text {out }}\right\rangle$ :

$$
\begin{aligned}
|f\rangle_{\text {in }} & =\exp \left[\frac{1}{2} \sum_{\vec{k}} \gamma_{\text {eff }}(\vec{k}) a_{\text {in }}^{\dagger}(\vec{k}) a_{\text {in }}^{\dagger}(-\vec{k})\right]\left|0_{\text {out }}\right\rangle \\
& =\exp \left[\frac{1}{2} \sum_{k} \kappa_{\text {eff }}(k) a_{\text {out }}^{\dagger}(\vec{k}) a_{\text {out }}(\vec{k})\right]|D\rangle
\end{aligned}
$$

where in the first line $\gamma_{\text {eff }}$ denotes the composite $\gamma$ :

$$
\gamma_{\mathrm{eff}}(k)=\frac{\beta^{*}(\vec{k})+f(\vec{k}) \alpha(\vec{k})}{\alpha^{*}(\vec{k})+f(\vec{k}) \beta(\vec{k})}
$$


and in the second line we have the relation $\kappa_{\text {eff }}=-\frac{1}{2} \log \left(-\gamma_{\text {eff }}\right)$, following similar arguments as for the ground state. Here $\alpha, \beta$ are given by (2.3). The state (2.20) is of the form of a gCC state. Can we get any gCC (with a given $\kappa(k)$ ) starting from a suitably chosen squeezing function $f$ ?

The answer is clearly yes. By solving (2.21) for $f$, we can clearly find an $f$ for any $\gamma_{\text {eff }}$. If we wish to generate a given $\kappa_{\text {eff }}(k) \equiv \kappa(k)$ in $(2.20)$, we must choose $\gamma_{\text {eff }}(k)=$ $-\exp [-2 \kappa(k)]$. This gives us

$$
f(\vec{k})=1-\frac{2 \omega_{\text {out }}}{\omega_{\text {in }} \tanh (\kappa(k))+\omega_{\text {out }}}
$$

This proves that we can prepare any gCC state, with a given $\kappa(k)$, from squeezed states:

$$
|f\rangle_{\text {in }}=\exp \left[\frac{1}{2} \sum_{k} \kappa(k) a_{\text {out }}^{\dagger}(\vec{k}) a_{\text {out }}(\vec{k})\right]|D\rangle=:|g C C\rangle
$$

Critical quench from specific squeezed states. Let us consider the case of critical quench $m_{\text {out }}=0$. We look at the following special states. From (2.22) it is clear that the choice $f(\vec{k})=f_{4}(k)=1-\frac{2 k}{\sqrt{k^{2}+m^{2}} \tanh \left(\kappa_{2} k+\kappa_{4} k^{3}\right)+k}$ leads to a state with only two non-zero tunable parameters $\kappa_{2}$ and $\kappa_{4}$

$$
|\psi(0)\rangle=\left|f_{4}\right\rangle=\exp \left[-\kappa_{2} H-\kappa_{4} Q_{4}\right]|D\rangle \equiv\left|g C C_{4}\right\rangle
$$

we call this the $g C C_{4}$ state because it is characterized by only two charges $Q_{2}$ and $Q_{4}$; we will find that the equilibrium state describing asymptotic correlators in $\left|g C C_{4}\right\rangle$ is a grand canonical ensemble characterized by a temperature and one chemical potential. Further if $\kappa_{4}$ is also zero, i.e. $f(\vec{k})=f_{2}(k)=1-\frac{2 k}{\sqrt{k^{2}+m^{2}} \tanh \left(\kappa_{2} k\right)+k}$ then we end up with the CC state

$$
|\psi(0)\rangle=\left|f_{4}\right\rangle=\exp \left[-\kappa_{2} H\right]|D\rangle \equiv|C C\rangle
$$

Note that $\kappa_{2}$ here is not related to the mass parameter $m$ unlike in $(2.9)$ where $\kappa_{2}=1 / m$.

\subsubsection{The squeezed state 2-point function}

The 2-point function in the squeezed state is the same as in the ground state with $\alpha$ and $\beta$ replaced by $\alpha_{\text {eff }}$ and $\beta_{\text {eff }}$ above. The 2-point function in the general squeezed state (2.23) is given by

$$
\begin{aligned}
& \left\langle g C C\left|\phi\left(\vec{x}_{1}, t_{1}\right) \phi\left(\vec{x}_{2}, t_{2}\right)\right| g C C\right\rangle \\
& =\int \frac{d^{d} k}{(2 \pi)^{d}} \frac{e^{i \vec{k} \cdot\left(\overrightarrow{x_{1}}-\overrightarrow{x_{2}}\right)}}{2 \sqrt{k^{2}+m_{\text {out }}^{2}}} \operatorname{csch}(2 \kappa(k))\left[\cos \left(\sqrt{k^{2}+m_{\text {out }}^{2}}\left(t_{1}-t_{2}\right)+2 i \kappa(k)\right)-\right. \\
& \left.\cos \left(\sqrt{k^{2}+m_{\text {out }}^{2}}\left(t_{1}+t_{2}\right)\right)\right]
\end{aligned}
$$

When $t_{1}=t_{2}=t$ this is

$$
\begin{aligned}
& \left\langle g C C\left|\phi\left(\vec{x}_{1}, t\right) \phi\left(\vec{x}_{2}, t\right)\right| g C C\right\rangle \\
& \quad=\int \frac{d^{d} k}{(2 \pi)^{d}} \frac{e^{i \vec{k} \cdot\left(\overrightarrow{x_{1}}-\vec{x}_{2}\right)}}{2 \sqrt{k^{2}+m_{\text {out }}^{2}}}\left[\operatorname{coth}(2 \kappa(k))-\cos \left(2 t \sqrt{k^{2}+m_{\text {out }}^{2}}\right) \operatorname{csch}(2 \kappa(k))\right]
\end{aligned}
$$




\begin{tabular}{|c|c|c|c|c|}
\hline post-quench state & $|g C C\rangle$ & $\left.|C C\rangle\right|_{m_{\text {out }}=0}$ & $\left.\left|g C C_{4}\right\rangle\right|_{m_{\text {out }}=0}$ & $\left|0_{\text {in }}\right\rangle$ \\
\hline form of $\kappa(k)$ & $\kappa(k)$ & $\kappa_{2} k$ & $\kappa_{2} k+\kappa_{4} k^{2}$ & $\frac{1}{2} \log \left(\frac{\sqrt{k^{2}+m^{2}}+\sqrt{k^{2}+m_{\text {out }}^{2}}}{\sqrt{k^{2}+m^{2}}-\sqrt{k^{2}+m_{\text {out }}^{2}}}\right)$ \\
\hline
\end{tabular}

Table 2. Ground states and specific squeezed states as examples of gCC states. The $\kappa(k)$ for the ground state (last column) is reproduced from (2.8). This table paves way for a uniform discussion all post-quench states discussed in this paper.

For the equal-time two-point function of $\partial_{t} \phi$, we apply to $(2.26)$ the rule $-f^{\prime \prime}(0)+g^{\prime \prime}(2 t)$ mentioned above (2.18), which gives us

$$
\begin{aligned}
& \left\langle g C C\left|\partial_{t} \phi\left(\vec{x}_{1}, t\right) \partial_{t} \phi\left(\vec{x}_{2}, t\right)\right| g C C\right\rangle \\
& \quad=\int \frac{d^{d} k}{(2 \pi)^{d}} \frac{e^{i \vec{k} \cdot\left(\overrightarrow{x_{1}}-\overrightarrow{x_{2}}\right)}}{2} \sqrt{k^{2}+m_{\text {out }}^{2}}\left[\operatorname{coth}(2 \kappa(k))+\cos \left(2 t \sqrt{k^{2}+m_{\text {out }}^{2}}\right) \operatorname{csch}(2 \kappa(k))\right]
\end{aligned}
$$

For the two-point function of spatial derivatives, these derives act only on the exponential term, leading to

$$
\begin{aligned}
& \left\langle g C C\left|\partial_{i} \phi\left(\vec{x}_{1}, t\right) \partial_{i} \phi\left(\vec{x}_{2}, t\right)\right| g C C\right\rangle \\
& \quad=\int \frac{d^{d} k}{(2 \pi)^{d}} \frac{k^{2} e^{i \vec{k} \cdot\left(\overrightarrow{x_{1}}-\overrightarrow{x_{2}}\right)}}{2 \sqrt{k^{2}+m_{\text {out }}^{2}}}\left[\operatorname{coth}(2 \kappa(k))-\cos \left(2 t \sqrt{k^{2}+m_{\text {out }}^{2}}\right) \operatorname{csch}(2 \kappa(k))\right]
\end{aligned}
$$

Note that the time-dependent parts of the above correlators, calling them $\langle\ldots\rangle_{g C C}^{(t d)}$, satisfy the relation

$$
\left\langle\partial_{i} \phi\left(\vec{x}_{1}, t\right) \partial_{i} \phi\left(\vec{x}_{2}, t\right)\right\rangle_{g C C}^{(t d)}+\left\langle\partial_{t} \phi\left(\vec{x}_{1}, t\right) \partial_{t} \phi\left(\vec{x}_{2}, t\right)\right\rangle_{g C C}^{(t d)}+m_{\text {out }}^{2}\left\langle\phi\left(\vec{x}_{1}, t\right) \phi\left(\vec{x}_{2}, t\right)\right\rangle_{g C C}^{(t d)}=0
$$

\section{3 gCC correlators encompass all cases}

As is clear from the foregoing discussion, all the particular post-quench states $|\psi(0)\rangle$ mentioned above, specifically $|C C\rangle,\left|g C C_{4}\right\rangle$, and $\left|0_{\text {in }}\right\rangle$, are examples of $|g C C\rangle$ states, with specific $\kappa(k)$ as in table 2. Consequently, the above formula (2.27), and the other related two-point functions, give the corresponding two-point functions in all these different cases. In particular, using the form of $k(k)$ (2.8) for ground state quench, one can recover all results of section 2.1.1 from those of section 2.2.1, and in particular recover (2.17) from (2.27) (after all, the ground state is a special squeezed state!). This implies, in principle, that if we derive some results on thermalization and relaxation for the general gCC states, using (2.27), we need not consider the above particular cases separately. However because each of these cases is significant on its own, we find it useful to often state the results separately.

\section{Time-dependence of two-point functions}

In this section, we will consider in more detail the time-dependent parts of the two-point functions discussed in the previous section. Since we are dealing with free field theories, all 
correlators are related to the basic two point function $\left\langle\psi(0)\left|\phi\left(x_{1}, t_{1}\right) \phi\left(x_{2}, t_{2}\right)\right| \psi(0)\right\rangle$. The primary goal of this paper is to study the long time behaviour of this quantity. We will find that it asymptotes ("thermalizes") to the corresponding observable in a GGE; we will find the characterization of the GGE and find the rate of approach to equilibrium. It is easy to generalize these results to multi-point functions, by Wick's theorem. It is also straightforward to compute correlators of composite operators from the above two-point function by appropriate regularization procedures.

In this section we will analytically evaluate the various 2-point functions (2.17), (2.18), (2.27) and (2.28) (note that the first two are a special case of the last two). We will indicate the main steps and list the results, leaving some details to the various appendices.

\subsection{General remarks}

Note that the various two-point functions mentioned in the previous paragraph are all of the form

$$
\int \frac{d^{d} k}{(2 \pi)^{d}} e^{i \vec{k} \cdot \vec{r}}\left[F(k)+G(k) \cos \left(2 t \omega_{k}\right)\right], \quad \omega_{k}=\sqrt{k^{2}+m_{\text {out }}^{2}}
$$

where $\vec{r}=\vec{x}_{1}-\vec{x}_{2}$. For correlators of the $\langle\phi \phi\rangle$ type,

$$
F(k)=\frac{1}{2 \omega_{k}} \operatorname{coth}(2 \kappa(k)), \quad G(k)=-\frac{1}{2 \omega_{k}} \operatorname{csch}(2 \kappa(k))
$$

To proceed further, we write the vector $\vec{k}$ in polar coordinates $\left(k, \theta, \varphi_{1}, \ldots, \varphi_{d-2}\right)$, oriented such that $\vec{r}$ points to the 'north pole' which ensures $\vec{k} \cdot \vec{r}=k r \cos \theta$; the $\varphi_{i}$ parameterize a unit sphere $S^{d-2}$ (we assume $d>1$ here). With this, the above integral becomes

$$
\begin{aligned}
& \int_{0}^{\infty} d k\left[F(k)+G(k) \cos \left(2 t \sqrt{k^{2}+m_{\text {out }}^{2}}\right)\right] A_{d}(k, r) \\
& A_{d}(k, r):=k^{d-1} \frac{\Omega_{d-2}}{(2 \pi)^{d}} \int_{0}^{\pi} d \theta e^{i k r \cos \theta}(\sin \theta)^{d-2}
\end{aligned}
$$

where $\Omega_{d-2}=\frac{2 \pi^{(d-1) / 2}}{\Gamma((d-1) / 2)}$ is the volume of $S^{d-2}$.

Angular integrals. The angular integration in (3.4) can be exactly done, which gives

$$
A_{d}(k, r)=k^{d-1} 2^{1-d} \pi^{-\frac{d}{2}}{ }_{0} \tilde{F}_{1}\left(\frac{d}{2} ;-\frac{1}{4} k^{2} r^{2}\right)
$$

The regularized Hypergeometric function ${ }_{0} \tilde{F}_{1}$ is some combination of trigonometric functions $\sin (k r)$ and $\cos (k r)$ in odd $d$ while it is some Bessel function in even $d$. The specific forms of $A_{d}(k, r)$ for various dimensions $d$ are tabulated in table 3 .

Recursion relations for 2-point functions. The structure of the integrals (3.1) occurring in the various correlators defined above makes it possible to connect them across dimensions through recursion relations(see appendix B for details). ${ }^{11}$ From a correlator in

\footnotetext{
${ }^{11}$ Similar recursion relations appear in a somewhat different context in [45].
} 


\begin{tabular}{|c|c|}
\hline number of spatial dimensions $d$ & $A_{d}(k, r)$ \\
\hline $1^{a}$ & $\frac{1}{\pi} \cos (k r)$ \\
\hline 2 & $\frac{k J_{0}(k r)}{2 \pi}$ \\
\hline 3 & $\frac{k \sin (k r)}{2 \pi^{2} r}$ \\
\hline 4 & $\frac{k^{2} J_{1}(k r)}{4 \pi^{2} r}$ \\
\hline 5 & $\frac{k(\sin (k r)-k r \cos (k r))}{4 \pi^{3} r^{3}}$ \\
\hline
\end{tabular}

${ }^{a}$ We added $d=1$ here for uniformity, using $\int_{-\infty}^{\infty} \frac{d k}{2 \pi} e^{i k x} f(|k|)=\int_{0}^{\infty} d|k| \frac{1}{\pi} \cos (|k||x|) f(|k|)$.

Table 3. The angular integral $A_{d}(k, r)$, (see eq. (3.5)) in the first few dimensions.

$d+1$ spacetime dimensions, one can obtain the correlator in $(d+2)+1$ dimensions by acting with a particular differential operator. This relation holds for both even and odd $d>1$ (here $t_{-}=t_{1}-t_{2}$ and $\left.t_{+}=t_{1}+t_{2}\right)$.

$$
\left\langle\phi\left(x_{1}, t_{1}\right) \phi\left(x_{2}, t_{2}\right)\right\rangle^{(d+2)}=\frac{\Omega_{d-2}}{4 \pi^{2} \Omega_{d-4}}\left(-\partial_{t_{-}}^{2}-\partial_{t_{+}}^{2}+m_{\text {out }}^{2}+\partial_{r}^{2}\right)\left\langle\phi\left(x_{1}, t_{1}\right) \phi\left(x_{2}, t_{2}\right)\right\rangle^{(d)}, \quad d>1
$$

where $\langle\ldots\rangle^{(d)}$ denotes an expectation value, in a $d+1$ dimensional theory, in a general post-quench state.

For the special case of equal time correlators which is our main focus, the recursion relation becomes

$$
\left\langle\phi\left(x_{1}, t_{1}\right) \phi\left(x_{2}, t_{2}\right)\right\rangle^{(d+2)}=\frac{\Omega_{d-2}}{4 \pi^{2} \Omega_{d-4}}\left(-\partial_{t_{+}}^{2}+m_{\text {out }}^{2}+\partial_{r}^{2}\right)\left\langle\phi\left(x_{1}, t_{1}\right) \phi\left(x_{2}, t_{2}\right)\right\rangle^{(d)}, d>1
$$

A similar relation holds for the equilibrium correlators. In an arbitrary GGE, we have the following recursion relation ( $t$ here is same as $\left.t_{-}\right)$

$$
\langle\phi(\vec{r}, t) \phi(0,0)\rangle_{\mu(k)}^{(d)}=\frac{\Omega_{d-2}}{4 \pi^{2} \Omega_{d-4}}\left(-\partial_{t}^{2}+m_{\text {out }}^{2}+\partial_{r}^{2}\right)\langle\phi(\vec{r}, t) \phi(0,0)\rangle_{\mu(k)}^{(d-2)}
$$

The $1+1$ dimensional 2-pt function is not connected to its $3+1$ dimensional counterpart in the above fashion, since in one dimensional space there is no angular coordinate which is crucial for this recursion relation to work (see appendix B). Nevertheless, there is a different, specific relation connecting the two:

$$
\left\langle\phi\left(x_{1}, t_{1}\right) \phi\left(x_{2}, t_{2}\right)\right\rangle^{(3+1)}=-\frac{1}{2 \pi r} \partial_{r}\left\langle\phi\left(x_{1}, t_{1}\right) \phi\left(x_{2}, t_{2}\right)\right\rangle^{(1+1)}
$$

which follows straightforwardly, by inspection.

Time-dependence. As remarked before, there is a time-dependent part and a timeindependent part of the generic equal time two-point function (3.1). We will come back to an analysis of the time-independent part in section 4 where we show that this part exactly matches the corresponding two-point function in a GGE (generalized Gibbs ensemble). 
In the remainder of the section, we will therefore consider only the time-dependent, non-equilibrium, part. We will show that this part decays to zero at large times, which amounts to a proof of thermalization, and we will derive the form of the decay. It will also be convenient, for this purpose, to differentiate between massive quench $m_{\text {out }} \neq 0$ and critical quench $m_{\text {out }}=0$. This is because in the latter case, the post-quench theory is conformal and we expect, and indeed verify, that this theory will have special properties as compared to the massive case. We will comment later (see section 3.4) why we cannot obtain results for the critical quench by simply taking the $m_{\text {out }} \rightarrow 0$ limit of the power series expansion for $m_{\text {out }} \neq 0$.

\subsection{Critical quench correlators}

As mentioned above, we will mainly focus on the time-dependent parts of the 2-point functions, while the time-independent parts will be considered in section 4 .

\subsubsection{Time-dependent part of the $\langle\phi \phi\rangle$ correlator}

Let us put $m_{\text {out }}=0$. To keep the discussion general, let us consider the general gCC correlator (2.27) (as emphasized in section 2.3, we can infer about the specific cases of ground states and the squeezed states from here). In the notation of (3.3), we now have

$$
F(k)=\frac{1}{2 k} \operatorname{coth}(2 \kappa(k)), G(k)=-\frac{1}{2 k} \operatorname{csch}(2 \kappa(k)), \cos \left(2 t \sqrt{k^{2}+m_{\mathrm{out}^{2}}}\right)=\cos (2 k t)
$$

The functions $\kappa(k)$ are tabulated for the special cases of interest in table 2, where for the ground state, we now have

$$
\kappa(k)=\frac{1}{2} \log \left(\frac{\sqrt{k^{2}+m^{2}}+k}{\sqrt{k^{2}+m^{2}}-k}\right)
$$

which has an expansion in odd powers of $k$, as mentioned above (2.9). Following this, we will focus, in the case of critical quench, only on $g C C$ states with $\kappa(k)$ which have an expansion in odd powers of $k$. The $\kappa(k)$ for the $\mathrm{CC}$ and $\mathrm{gCC}_{4}$ states, of course, satisfy this by definition.

With this assumption, the functions $F(k), G(k)$ are even in $k$.

\subsubsection{Large time behaviour for odd $d$}

Note from the table $3, A_{d} k, r$ that for odd $d$ are even functions of $k$. Using the evenness of $F(k), G(k)$, we find that the integrand in (3.3) is even, which allows us to extend the integration contour to the entire real line (for even $d$, this step is not allowed, hence we will do something else). If one can close the contour on the upper or the lower half plane the integral can be evaluated by the method of residues. To see how it works, let us take the example of $d=3$ (the procedure described below is similar to the case of $d=1$ [35]; and in special cases, where exact results are known in $d=1$, the 3 -dimensional results can be derived by using (3.9)). For more details see appendix C.2. 
For the critical gCC correlator at $d=3$, we get

$$
\begin{aligned}
& \left\langle g C C\left|\phi\left(x_{1}, t\right) \phi\left(x_{2}, t\right)\right| g C C\right\rangle \\
& =\frac{1}{4 \pi^{2} r} \int_{0}^{\infty} d k \sin (k r)[\operatorname{coth}(2 \kappa(k))-\operatorname{csch}(2 \kappa(k)) \cos (2 k t)] \\
& =\frac{1}{8 \pi^{2} i r} \int_{-\infty}^{\infty} d k e^{i k r}[\operatorname{coth}(2 \kappa(k))-\operatorname{csch}(2 \kappa(k)) \cos (2 k t)] \\
& =\frac{1}{8 \pi^{2} i r} \int_{-\infty}^{\infty} d k[\underbrace{\frac{\cosh (2 \kappa(k)) e^{i k r}}{\sinh (2 \kappa(k))}}_{\text {A }}-\underbrace{\frac{e^{i k(r+2 t)}}{2 \sinh (2 \kappa(k))}}_{\text {B }}-\underbrace{\frac{e^{i k(r-2 t)}}{2 \sinh (2 \kappa(k))}}_{\text {C }}]
\end{aligned}
$$

Let us focus on the time-dependent terms $\mathrm{B}$ and $\mathrm{C}$ (the time-independent term $\mathrm{A}$ is described later on, and in appendix C.2). We will also assume large $t$ so that $2 t>r$. Then from the asymptotic behaviour of functions $\kappa(k)$ in the table 2, one can see that the functions $\kappa(k)$ grow as $k \rightarrow \infty$, in a way that ensures that the integrals are convergent, and for large $t$ (so that $2 t>r$ ) it is possible to close the contour for the integral B on the UHP and for $\mathrm{C}$ on the LHP. At large times $t$, the slowest transient is given by the pole of the integrand singularity nearest to the origin (in the UHP for B and LHP for C; the pole at the origin itself cancels between the terms B and C; see appendix C.2, especially figure 6). It is easy to see that for a $\mathrm{gCC}$ with a finite number of chemical potentials, the nearest such singularity is a pole. This is given by the relevant zero of $\sinh (2 \kappa(k))$, or, equivalently by the corresponding root of

$$
2 \kappa(k)= \pm i \pi
$$

The root discussed above turns out to be of the form as $k=k_{1} \pm i k_{0}, k_{0}>0$; here we choose the + and - signs respectively for the $\mathrm{B}$ and $\mathrm{C}$ terms; the value of $k_{1}$ may be zero, positive or negative. By applying residue calculus, we find that the slowest decay at large $t$ of the $\phi \phi$ correlator is given by

$$
b \exp \left[-k_{0}(t+2 r)\right]+c \exp \left[-k_{0}(t-2 r)\right]
$$

For non-zero $k_{1}$ this comes multiplied by an oscillatory term, of time period $2 \pi / k_{1}$. In case of the CC state, the exact time-dependence can be calculated by summing over the poles (see appendix C.2 for details). The exact two-point function for the CC state as well as for the ground state can also be derived from the their $1+1$ dimensional counterparts found in [35] using the recursion relation (3.9) (see appendix C.2). The net result of all this is that at large times the time-dependent part of the critical $\langle\phi \phi\rangle$ fall off exponentially, as in (3.14); these are tabulated in table 4.

In case of a gCC with an infinite number of chemical potentials, such as when the gCC is the ground state, the poles can merge into a branch cut (see also a similar discussion in [35]). The structure (3.14) is retained even here, with additional power-law prefactors. Details of the above procedure for the $\mathrm{CC}, \mathrm{gCC}_{4}$ and the ground state, are worked out in appendix C.2. Note that although for specificity we had taken the example of $d=3$ here, the arguments go through for $d=5$ or any higher odd space dimensions. 


\begin{tabular}{|l|l|l|}
\hline \multicolumn{2}{|c|}{$\left\langle\psi(0)\left|\phi\left(\overrightarrow{x_{1}}, t\right) \phi\left(\overrightarrow{x_{2}}, t\right)\right| \psi(0)\right\rangle$} \\
\hline$|\psi(0)\rangle$ & $\mathbf{d}=\mathbf{3}$ & $\mathbf{d}=\mathbf{4}$ \\
\hline $\begin{array}{l}\text { Ground } \\
\text { state }\end{array}$ & $\frac{-m^{2}}{16 \pi^{5 / 2} \sqrt{m t}} e^{-2 m t}\left(1+\mathcal{O}(m r)^{2}\right)\left(1+\mathcal{O}(m t)^{-1}\right)$ & $\frac{m}{128 \pi^{2}} t^{2}+\mathcal{O}\left(\frac{1}{t^{4}}\right)$ \\
\hline $\begin{array}{l}\text { CC } \\
\text { state }\end{array}$ & $\frac{-1}{16 \kappa_{2}^{2}} e^{-\pi t / \kappa_{2}}\left(1+\mathcal{O}\left(\frac{r}{\kappa_{2}}\right)^{2}\right)+\mathcal{O}\left(e^{-2 \pi t / \kappa_{2}}\right)$ & $\frac{1}{128 \pi^{2} \kappa_{2}} \frac{1}{t^{2}}+\mathcal{O}\left(\frac{1}{t^{4}}\right)$ \\
\hline $\begin{array}{l}\text { gCC } \\
\text { state }\end{array}$ & $\frac{-\left(1+\pi^{2} \overline{\kappa_{4}}+\cdots\right)}{16 \kappa_{2}^{2}} e^{-\frac{\pi t}{\kappa_{2}}\left(1+\frac{\pi^{2}}{4} \kappa_{4}+\cdots\right)}\left(1+\mathcal{O}\left(\frac{r}{\kappa_{2}}\right)^{2}\right)+\mathcal{O}\left(e^{-2 \pi t / \kappa_{2}}\right)$ & $\frac{1}{128 \pi^{2} \kappa_{2}} \frac{1}{t^{2}}+\frac{3 r^{2}+16 \kappa_{2}^{2}+24 \kappa_{4} / \kappa_{2}}{2048 \pi^{2} \kappa_{2}} \frac{1}{t^{4}}+\mathcal{O}\left(\frac{1}{t^{6}}\right)$ \\
\hline
\end{tabular}

Table 4. Time-dependent part of 2-point function $\left\langle\psi(0)\left|\phi\left(\overrightarrow{x_{1}}, t\right) \phi\left(\overrightarrow{x_{2}}, t\right)\right| \psi(0)\right\rangle$ in critical quench for $d=3$ (left column) and $d=4$ (right column). Here $r=\left|\overrightarrow{x_{1}}-\overrightarrow{x_{2}}\right|$. Note that for even $d$, e.g. $d=4$, the leading large $t$ dependence is the same in all three types of post-quench state $|\psi(0)\rangle$, with the identification $m=1 / \kappa_{2}$ (see (2.9)). Further the gCC answer tells us that since the higher $\kappa_{i}$ 's for $i>2$ show up in subleading terms, the higher conserved charges do not affect the leading transient towards thermalization. However, as we will see later, the time-independent part of these two-point functions reflects an equilibrium given by a GGE which is characterized by all the $\kappa_{i}$ parameters.

Since we are interested in the leading approach to equilibrium for simplicity, in the tables we only display the correlators in the limit $m t \gg 1 \gg m r$ or equivalently $\frac{t}{\kappa_{2}} \gg 1 \gg$ $\frac{r}{\kappa_{2}}$. The ellipsis in the $\mathrm{gCC}_{4}$ correlator represents $\mathcal{O}\left(\bar{\kappa}_{4}^{2}\right)$ terms.

General odd $\boldsymbol{d}$ result. From the recursion relation (3.6) it follows that for all odd $d \geq 3$, the slowest decay at large time of the time-dependent part of the $\phi \phi$ correlator is given by replacing (3.14) with

$$
b_{d} \exp \left[-k_{0}(t+2 r)\right]+c_{d} \exp \left[-k_{0}(t-2 r)\right]
$$

where $k_{0}$ has the same value discussed above and is independent of dimension $d$. The constants $b_{d}, c_{d}$ depend on the dimension $d$. The expression above may come with a possible oscillatory part as explained below (3.14).

\subsubsection{Large time behaviour for even $d$}

Here the angular function, $A_{d}(k, r)$ for even $d$ are odd functions of $k$. Therefore, unlike in odd $d$, we cannot extend the integration contour to the entire real line and apply the method of residues. Fortunately there does exist a different approach in this case. Let us take the example of $d=4$ to illustrate the method. As in the case of odd $d$, we allow the most general $\kappa(k)=\sum_{i=1}^{\infty} \kappa_{2 i} k^{2 i-1}$ which grows at infinity such that the integral is convergent. The critical gCC correlator at $d=4$ is

$$
\left\langle g C C\left|\phi\left(x_{1}, t\right) \phi\left(x_{2}, t\right)\right| g C C\right\rangle=\frac{1}{8 \pi^{2} r} \int_{0}^{\infty} d k k J_{1}(k r)[\operatorname{coth}(2 \kappa(k))-\operatorname{csch}(2 \kappa(k)) \cos (2 k t)]
$$

In the notation of equation (3.3), $G(k)=-\operatorname{csch}(2 \kappa(k)) /(2 k)$. As before we are interested in large t. Consider the following scaling

$$
k \rightarrow p=2 k t
$$


and rewrite the time-dependent part in terms of this new dimensionless momenta $p$

$$
\begin{aligned}
& -\frac{1}{8 \pi^{2} r} \int_{0}^{\infty} d k k J_{1}(k r) \operatorname{csch}(2 \kappa(k)) \cos (2 k t) \\
& =-\frac{1}{32 \pi^{2} r} \frac{1}{t^{2}} \int_{0}^{\infty} d p p J_{1}\left(\frac{p r}{2 t}\right) \operatorname{csch}\left(2 \kappa\left(\frac{p}{2 t}\right)\right) \cos (p) \\
& =-\frac{1}{64 \pi^{2} r} \frac{1}{t^{2}}\left\{\int_{0}^{\infty(1+i \epsilon)} d p p J_{1}\left(\frac{p r}{2 t}\right) \operatorname{csch}\left(2 \kappa\left(\frac{p}{2 t}\right)\right) e^{i p}+\int_{0}^{\infty(1-i \epsilon)} d p p J_{1}\left(\frac{p r}{2 t}\right) \operatorname{csch}\left(2 \kappa\left(\frac{p}{2 t}\right)\right) e^{-i p}\right\} \\
& =-\frac{1}{256 \pi^{2} \kappa_{2}}\left[\frac{I(1)}{t^{2}}-\frac{\left(16 \kappa_{2}^{3}+24 \kappa_{4}+3 \kappa_{2} r^{2}\right)}{96 \kappa_{2} t^{4}} I(3)+\mathcal{O}\left(t^{-6}\right)\right]
\end{aligned}
$$

In the second line we have rotated the contour anti-clockwise for $e^{i p}$ and clockwise for $e^{-i p}$. We can do this because the integrand $J_{1}(k r) \operatorname{csch}\left(2 \kappa_{2} k\right)$ vanishes on the arc at infinity due to the exponential damping in the $\operatorname{csch}(2 \kappa(k))$ as $k \rightarrow \infty$. In the last line we have Taylor expanded the integrand at large $t$. We have also introduced the integrals $I(n)$ defined by

$$
\begin{aligned}
I(n) & =\lim _{\epsilon \rightarrow 0} \int_{0}^{\infty(1+i \epsilon)} d p e^{i p} p^{n}+\lim _{\epsilon \rightarrow 0} \int_{0}^{\infty(1-i \epsilon)} d p e^{-i p} p^{n} \\
& =\lim _{\epsilon \rightarrow 0} \int_{0}^{\infty} d p_{+} e^{i p_{+}} p_{+}^{n}+\lim _{\epsilon \rightarrow 0} \int_{0}^{\infty} d p_{-} e^{-i p-} p_{-}^{n} \\
& =\lim _{\epsilon \rightarrow 0} \int_{0}^{\infty} d p\left(e^{p(i-\epsilon)}+e^{-p(i+\epsilon)}\right) p^{n}+\mathcal{O}(\epsilon) \\
& =\lim _{\epsilon \rightarrow 0}\left(\partial_{-\epsilon}\right)^{n} \int_{0}^{\infty} d p\left(e^{p(i-\epsilon)}+e^{-p(i+\epsilon)}\right)=\lim _{\epsilon \rightarrow 0}\left(\partial_{-\epsilon}\right)^{n}\left(\frac{2 \epsilon}{1+\epsilon^{2}}\right) \\
I(n) & = \begin{cases}(-1)^{(n+1) / 2} 2 \times n ! & \mathrm{n} \text { is odd } \\
0 & \mathrm{n} \text { is even }\end{cases}
\end{aligned}
$$

where in the second line we have introduced $p_{ \pm}=p(1 \pm i \epsilon)$. Note that in spite of the growth of the $I(n)$ with $n$, because of the even more strongly convergent power series expansion for the product of the Bessel and cosech functions, the equation (3.17) has a convergent expansion in $1 / t^{2}$ for large enough $t$.

Notice that $\kappa_{4}$ shows up only from the $O\left(t^{-4}\right)$ onwards. Similarly $\kappa_{6}$ starts appearing only from the $\mathcal{O}\left(t^{-6}\right)$ term; explicitly that term is

$$
\frac{64\left(14 \kappa_{2}^{6}-30 \kappa_{2}^{3} \kappa_{4}-45 \kappa_{2} \kappa_{6}+45 \kappa_{4}^{2}\right)+15 \kappa_{2}^{2} r^{4}+120 r^{2}\left(2 \kappa_{2}^{4}+3 \kappa_{2} \kappa_{4}\right)}{46080 \kappa_{2}^{2} t^{6}} I(5)
$$

Having calculated $I(n)$ 's, we find that the time-dependent part of the gCC 2-point function goes as

$$
-\frac{1}{8 \pi^{2} r} \int_{0}^{\infty} d k k J_{1}(k r) \operatorname{csch}(2 \kappa(k)) \cos (2 k t)=\frac{1}{128 \pi^{2} \kappa_{2}} \frac{1}{t^{2}}+\frac{3 r^{2}+16 \kappa_{2}^{2}+24 \kappa_{4} / \kappa_{2}}{2048 \pi^{2} \kappa_{2}} \frac{1}{t^{4}}+\mathcal{O}\left(t^{-6}\right)
$$

The effect of $\kappa_{4}$ is suppressed by $\mathcal{O}\left(t^{-2}\right)$ relative to the leading term and therefore it makes no difference to the leading power law fall off in the large $t$ limit. The same is true for higher $\kappa_{n}$ 's, as they are even more suppressed. 


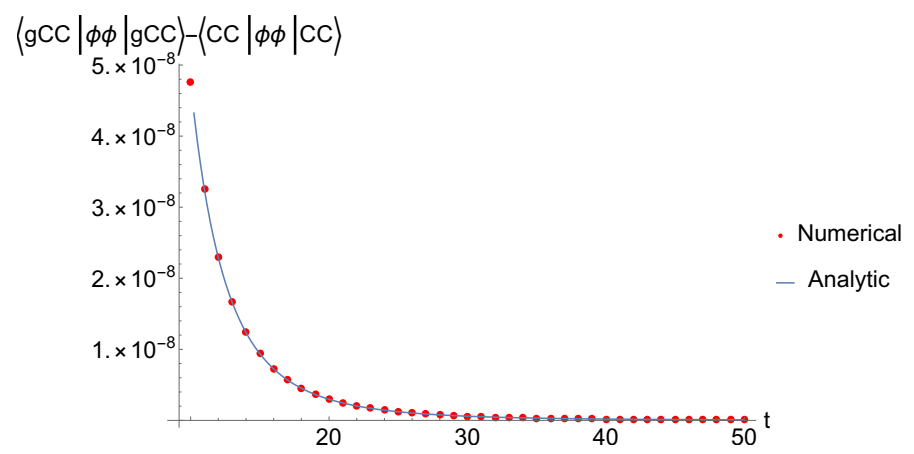

Figure 2. We plot the $\mathrm{gCC}_{4}$ equal-time correlator after subtracting off the $\mathrm{CC}$ part. This gives us the contribution depending solely on $\kappa_{4}$. There is near perfect match between the analytic and numerical results. (Numerics done for values $r=0.1, \kappa_{2}=0.25$ and $\kappa_{4}=0.025$ ).

The equation (3.19) can be verified against explicit numerical integration of the momentum integral. In practical terms, the subleading term, of order $1 / t^{4}$, is possible to read off by first also calculate the time-dependent part in the $\mathrm{gCC}_{4}$ state numerically and see exact agreement in subleading $\mathcal{O}\left(t^{-4}\right)$ behaviour after subtracting off the $\kappa_{2}$-dependent part (figure 2).

General even $\boldsymbol{d}$ result. From the recursion relation (3.6) it follows that for all $d \geq 4$, the time-dependent part of the $\phi \phi$ correlator decays as (up to time-independent factors)

$$
\frac{1}{t^{d-2}}
$$

The $d=2$ case is discussed separately below (see table 6) for a summary and also appendix C.1 for details).

One might wonder if the same reasoning would have given us the answer in odd $d$, then we would not have to do the contour integrals there. In $d=3$ for example, the time-dependent part of the 2-point function has even powers of $p$ (eq. (3.16))

$$
\begin{aligned}
- & \frac{1}{4 \pi^{2} r} \int_{0}^{\infty} d k \sin (k r) \operatorname{csch}\left(2 \kappa_{2} k\right) \cos (2 k t) \\
& =-\frac{1}{16 \pi^{2} \kappa_{2}} \int_{0}^{\infty} d p\left[\frac{1}{t}-\frac{\left(4 \kappa_{2}^{3}+6 \kappa_{4}+\kappa_{2} r^{2}\right) p^{2}}{24 \kappa_{2} t^{3}}+\mathcal{O}\left(t^{-5}\right)\right] \cos (p) \\
& =-\frac{1}{32 \pi^{2} \kappa_{2}}\left[\frac{I(0)}{t}-\frac{\left(4 \kappa_{2}^{3}+6 \kappa_{4}+\kappa_{2} r^{2}\right) I(2)}{24 \kappa_{2} t^{3}}+\mathcal{O}\left(t^{-5}\right)\right] \\
& =0
\end{aligned}
$$

What this means is that either the answer is zero or something non-perturbative, i.e. a function with no Taylor expansion at $t=\infty$. Since we already know the answer is an exponentially decaying function $e^{-\pi t / \kappa_{2}}$, we know it is the latter. The above argument elucidates the odd-even difference in the approach to thermalization. We give another geometric understanding in the next section 5 . 


\begin{tabular}{|l|l|l|}
\hline \multicolumn{2}{|c|}{$\left\langle\psi(0)\left|\partial_{t} \phi\left(\overrightarrow{x_{1}}, t\right) \partial_{t} \phi\left(\overrightarrow{x_{2}}, t\right)\right| \psi(0)\right\rangle$} \\
\hline$|\psi(0)\rangle$ & $\mathbf{d}=\mathbf{3}$ & $\mathbf{d}=\mathbf{4}$ \\
\hline $\begin{array}{l}\text { Ground } \\
\text { state }\end{array}$ & $\frac{-m^{4}}{16 \pi^{3 / 2} \sqrt{m t}} e^{-2 m t}\left(1+\mathcal{O}(m r)^{2}\right)\left(1+\mathcal{O}(m t)^{-1}\right)$ & $\frac{3 m}{256 \pi^{2}} \frac{1}{t^{4}}+\mathcal{O}\left(\frac{1}{t^{6}}\right)$ \\
\hline $\begin{array}{l}\text { CC } \\
\text { state }\end{array}$ & $\frac{-\pi^{2}}{64 \kappa_{2}^{4}} e^{-\pi t / \kappa_{2}}\left(1+\mathcal{O}\left(\frac{r}{\kappa_{2}}\right)^{2}\right)+\mathcal{O}\left(e^{-2 \pi t / \kappa_{2}}\right)$ & $\frac{3}{256 \pi^{2} \kappa_{2}} \frac{1}{t^{4}}+\mathcal{O}\left(\frac{1}{t^{6}}\right)$ \\
\hline $\begin{array}{l}\text { gCC } \\
\text { state }\end{array}$ & $\frac{-\pi^{2}\left(1+\frac{3 \pi^{2} \kappa_{4}}{64 \kappa_{2}^{4}}+\cdots\right)}{2} e^{-\frac{\pi t}{\kappa_{2}}\left(1+\frac{\pi^{2}}{4} \widetilde{\kappa_{4}}+\cdots\right)}\left(1+\mathcal{O}\left(\frac{r}{\kappa_{2}}\right)^{2}\right)+\mathcal{O}\left(e^{-2 \pi t / \kappa_{2}}\right)$ & $\frac{3}{256 \pi^{2} \kappa_{2}} \frac{1}{t^{4}}-\frac{15 r^{2}+80 \kappa_{2}^{2}+120 \kappa_{4}}{2048 \pi^{2} \kappa_{2} t^{6}}+\mathcal{O}\left(\frac{1}{t^{8}}\right)$ \\
\hline
\end{tabular}

Table 5. Time-dependent part of 2-point function $\left\langle\psi(0)\left|\partial_{t} \phi\left(\overrightarrow{x_{1}}, t\right) \partial_{t} \phi\left(\overrightarrow{x_{2}}, t\right)\right| \psi(0)\right\rangle$ in critical quench in $d=3$ in the left column and $d=4$ in the right column. Here $r=\left|\overrightarrow{x_{1}}-\overrightarrow{x_{2}}\right| \cdot|\psi(0)\rangle$ denotes the type of post-quench state.

\begin{tabular}{|l|l|l|}
\hline \multicolumn{3}{|c|}{$\left\langle\psi(0)\left|\partial_{t} \phi\left(\overrightarrow{x_{1}}, t\right) \partial_{t} \phi\left(\overrightarrow{x_{2}}, t\right)\right| \psi(0)\right\rangle$} \\
\hline $\begin{array}{l}\text { Ground } \\
\text { state }\end{array}$ & $\frac{m^{2}}{8 \pi^{1 / 2} \sqrt{m t}} e^{-2 m t}\left(1+\mathcal{O}(m r)^{2}\right)\left(1+\mathcal{O}(m t)^{-1}\right)$ & $\mathbf{d}=\mathbf{2}$ \\
\hline $\begin{array}{l}\text { CC } \\
\text { state }\end{array}$ & $\frac{\pi}{8 \kappa_{2}^{2}} e^{-\pi t / \kappa_{2}}\left(1+\mathcal{O}\left(\frac{r}{\kappa_{2}}\right)^{2}\right)+\mathcal{O}\left(e^{-2 \pi t / \kappa_{2}}\right)$ & $-\frac{m}{32 \pi t^{2}}+\mathcal{O}\left(\frac{1}{t^{4}}\right)$ \\
\hline $\begin{array}{l}\mathrm{gCC}_{4} \\
\text { state }\end{array}$ & $\frac{\pi\left(1+\pi \kappa_{4}+\cdots\right)}{8 \kappa_{2}^{2}} e^{-2 t\left(\frac{\pi}{2 \kappa_{2}}+\frac{\pi^{3} \kappa_{4}}{8 \kappa_{2}}+\cdots\right.}+\mathcal{O}\left(\frac{1}{t^{4}}\right)$ \\
\hline
\end{tabular}

Table 6. Time-dependent part of 2-point function $\left\langle\psi(0)\left|\partial_{t} \phi\left(\overrightarrow{x_{1}}, t\right) \partial_{t} \phi\left(\overrightarrow{x_{2}}, t\right)\right| \psi(0)\right\rangle$ in critical quench in $d=1$ in the left column and $d=2$ in the right column. Here $|\psi(0)\rangle$ denotes the type of post-quench state. $\left.r=\left|\overrightarrow{x_{1}}-\overrightarrow{x_{2}}\right|\right)$.

\subsection{4 $\partial_{i} \phi \partial_{i} \phi$ and $\partial_{t} \phi \partial_{t} \phi$ correlator}

As argued in section 2.1.1, the 2-point function of $\partial \phi$ is not independent of the 2-point function of $\phi$. As explained in that section, in $d=1$ and 2, our primary interest is in $\langle\partial \phi \partial \phi\rangle$. For $d=1$ (see [35] for more details) this quantity for the general gCC state can be obtained by using the equations (2.28), (3.3) and (3.4):

$$
\left\langle g C C\left|\partial_{t} \phi\left(x_{1}, t\right) \partial_{t} \phi\left(x_{2}, t\right)\right| g C C\right\rangle=\frac{1}{4 \pi} \int_{-\infty}^{\infty} d k e^{i k r} k[\operatorname{coth}(2 \kappa(k))+\operatorname{csch}(2 \kappa(k)) \cos (2 k t)]
$$

This expression can be evaluated in the same manner as described in section 3.2.2. Similarly for $d=2$, the time dependent part of the gCC 2-point function can be evaluated by again using equations (2.28), (3.3) and (3.4):

$$
\left\langle\partial_{t} \phi\left(\overrightarrow{x_{1}}, t\right) \partial_{t} \phi\left(\overrightarrow{x_{2}}, t\right)\right\rangle_{t d}=\frac{1}{4 \pi} \int_{0}^{\infty} d k e^{i k r} k^{2} J_{0}(k r) \cos (2 k t) \operatorname{csch}(2 \kappa(k))
$$

This expression can be evaluated in the same manner as described in section 3.2.3. We tabulate the results in table 6 .

\subsection{Massive quench correlators}

In the massive or non-critical quench the final mass $m_{\text {out }}$ is non-zero. Here we incorporate stationary phase approximation to perform the momentum integrals. 


\subsection{1 $\phi \phi$ correlator}

In the notation of (3.1), the time dependent part of 2-point function in the gCC state (2.27) can be written as

$$
\begin{aligned}
\int_{0}^{\infty} & d k G(k) \cos \left(2 t \sqrt{k^{2}+m_{\text {out }}^{2}}\right) A_{d}(k, r) \\
= & \frac{1}{2} \int_{0}^{\infty} d k G(k)\left(e^{i 2 t \sqrt{k^{2}+m_{\text {out }}^{2}}}+e^{-i 2 t \sqrt{k^{2}+m_{\text {out }}^{2}}}\right) A_{d}(k, r) \\
= & \int_{0}^{\infty} d k f_{d}(k, r) e^{i t w(k)}+\int_{0}^{\infty} d k f_{d}(k, r) e^{-i t w(k)} \\
= & I_{1}+I_{2}
\end{aligned}
$$

$G(k)=-\frac{\operatorname{csch}(2 \kappa(k))}{2 \sqrt{k^{2}+m_{\text {out }}^{2}}}$ for an arbitrary gCC state, while $G(k)=\frac{\left(m_{\text {out }}^{2}-m^{2}\right)}{4 \sqrt{k^{2}+m^{2}}\left(k^{2}+m_{\text {out }}^{2}\right)}$ for the ground state. $A_{d}(k, r)=k^{d-1} 2^{1-d} \pi^{-\frac{d}{2}}{ }_{0} \tilde{F}_{1}\left(\frac{d}{2} ;-\frac{1}{4} k^{2} r^{2}\right)$ is the angular function defined before (3.4) and $f_{d}(k, r)=\frac{1}{2} G(k) A_{d}(k, r)$. We consider the gCC states defined by

$$
\kappa(k)=\sum_{i=1}^{\infty} \kappa_{2 i-1} k^{2(i-1)}=\kappa_{1}+\kappa_{3} k^{2}+\kappa_{5} k^{4}+\cdots
$$

The reason for choosing only even powers of $k$ is motivated by only even powers appearing in eq. (2.10). Let $k=k_{0}$ be a stationary point of the integral $I_{1}$ (for which $w^{\prime \prime}\left(k_{0}\right)>0$ ) having the general form

$$
\begin{aligned}
& I_{1}(t)=m_{\text {out }}^{d-1} \int d k f(k) e^{i t w(k)} \underset{t \rightarrow i t}{\stackrel{\text { Wick rotate }}{\longrightarrow}} m_{\text {out }}^{d-1} \int d k f(k) e^{-t w(k)} \\
& =m_{\text {out }}^{d-1} \frac{e^{-t w_{0}}}{\sqrt{t}} \int d y\left(f_{0}+f_{0}^{\prime} \frac{y}{\sqrt{t}}+\frac{1}{2 !} f_{0}^{\prime \prime} \frac{y^{2}}{t}+\frac{1}{3 !} f_{0}^{\prime \prime \prime} \frac{y^{3}}{t^{3 / 2}}+\cdots\right) \times \\
& \exp -\left(\frac{w_{0}^{\prime \prime}}{2 !} y^{2}+\frac{w_{0}^{\prime \prime \prime}}{3 !} \frac{y^{3}}{\sqrt{t}}+\frac{w_{0}^{\prime \prime \prime \prime}}{4 !} \frac{y^{4}}{t}+\cdots\right) \\
& =m_{\text {out }}^{d-1} \frac{e^{-2 t}}{\sqrt{t}} \int d y\left(f_{0}+f_{0}^{\prime} \frac{y}{\sqrt{t}}+\frac{1}{2 !} f_{0}^{\prime \prime} \frac{y^{2}}{t}+\frac{1}{3 !} f_{0}^{\prime \prime \prime} \frac{y^{3}}{t^{3 / 2}}+\cdots\right) \exp -\left(y^{2}-\frac{1}{4} \frac{y^{4}}{t}+\cdots\right) \\
& =m_{\text {out }}^{d-1} \frac{e^{-2 t}}{\sqrt{t}} \int d y e^{-y^{2}}\left(f_{0}+f_{0}^{\prime} \frac{y}{\sqrt{t}}+\frac{1}{2 !} f_{0}^{\prime \prime} \frac{y^{2}}{t}+\frac{1}{3 !} f_{0}^{\prime \prime \prime} \frac{y^{3}}{t^{3 / 2}}+\cdots\right)\left(1+\frac{1}{4} \frac{y^{4}}{t}+\cdots\right)
\end{aligned}
$$

where we Wick rotate $t \rightarrow i t$ in the first line (when $w^{\prime \prime}\left(k_{0}\right)<0$ which is the case in $I_{2}$, we rotate in the opposite direction i.e, $t \rightarrow-i t)$. We expand both $f$ and $w$ around $k=k_{0}$ and introduce the variable $y=\sqrt{t}\left(k-k_{0}\right)$ in the second line. We are using compact notation to write $f_{0}=f\left(k_{0}\right), f_{0}^{\prime}=f^{\prime}\left(k=k_{0}\right)$, etc. and similarly for $w$. In the third line plug in $w(k)=\sqrt{k^{2}+1}$ and finally in the last line we have brought down all terms in the exponent except for $y^{2}$. The factor $m_{\text {out }}^{d-1}$ is pulled out to make rest of the integral dimensionless. So every quantity inside the integral is multiplied by appropriate power of $m_{\text {out }}$ to make it dimensionless.

$$
t \rightarrow m_{\text {out }} t ; \quad r \rightarrow m_{\text {out }} r ; \quad k \rightarrow k / m_{\text {out }} ; \quad m \rightarrow m / m_{\text {out }} .
$$


$G(k)$ is an even function of $k$, while $A_{d}(k, r)$ is an even function only in odd dimensions but an odd function in even dimensions. This means for even $d$, the integration is necessarily to be done for $k$ belonging to $[0, \infty)$. This has important consequences for us because the stationary point which is the solution of the equation $w^{\prime}\left(k_{0}\right)=0$ is exactly $k_{0}=0$. So for even $d$, the odd powers of $y$ will also contribute. Since the most contribution comes near $k=0$, we look at the behaviour of $f(k)$ near the origin

$$
f(k)=-\frac{\pi^{-\frac{d}{2}}}{2^{d+1} \Gamma\left(\frac{d}{2}\right)} \operatorname{csch}\left(2 \kappa_{1}\right) k^{d-1}+\frac{\operatorname{csch}\left(2 \kappa_{1}\right)\left(d+r^{2}+4 d \kappa_{3} \operatorname{coth}\left(2 \kappa_{1}\right)\right)}{2^{d+3} \pi^{\frac{d}{2}} \Gamma\left(\frac{d}{2}+1\right)} k^{d+1}+\mathcal{O}\left(k^{d+3}\right)
$$

Therefore the leading contribution comes from the $(d-1)$ th derivative i.e. $f_{0}^{(d-1)} \frac{y^{d-1}}{t^{(d-1) / 2}}$ which together with the $e^{-2 t} / \sqrt{t}$ goes as $t^{-d / 2}$. More explicitly, keeping track upto the sub-leading term in $I_{1}$

$$
\begin{aligned}
\frac{I_{1}(t)}{m_{\mathrm{out}}^{d-1}} \approx & -\frac{\operatorname{csch}\left(2 \kappa_{1}\right)}{2^{d+1} \pi^{\frac{d}{2}} \Gamma\left(\frac{d}{2}\right)} \frac{e^{-2 t}}{t^{d / 2}} \int_{0}^{\infty} d y e^{-y^{2}} y^{d-1}+\frac{\operatorname{csch}\left(2 \kappa_{1}\right)\left(d+r^{2}+4 d \kappa_{3} \operatorname{coth}\left(2 \kappa_{1}\right)\right)}{2^{d+3} \pi^{\frac{d}{2}} \Gamma\left(\frac{d}{2}+1\right)} \frac{e^{-2 t}}{t^{1+d / 2}} \\
& \times \int_{0}^{\infty} d y e^{-y^{2}} y^{d+1}-\frac{\operatorname{csch}\left(2 \kappa_{1}\right)}{2^{d+1} \pi^{\frac{d}{2}} \Gamma\left(\frac{d}{2}\right)} \frac{e^{-2 t}}{t^{d / 2}}\left(\frac{1}{4 t}\right) \int_{0}^{\infty} d y e^{-y^{2}} y^{d+3} \\
= & -\frac{\operatorname{csch}\left(2 \kappa_{1}\right)}{2^{d+2} \pi^{\frac{d}{2}}} \frac{e^{-2 t}}{t^{d / 2}}+\frac{\operatorname{csch}\left(2 \kappa_{1}\right)\left(d+r^{2}+4 d \kappa_{3} \operatorname{coth}\left(2 \kappa_{1}\right)\right)}{2^{d+4} \pi^{\frac{d}{2}}} \frac{e^{-2 t}}{t^{1+d / 2}}-\frac{d(d+2) \operatorname{csch}\left(2 \kappa_{1}\right)}{2^{d+6} \pi^{\frac{d}{2}}} \frac{e^{-2 t}}{t^{1+d / 2}} \\
= & -\frac{\operatorname{csch}\left(2 \kappa_{1}\right)}{2^{d+2} \pi^{\frac{d}{2}}} \frac{e^{i 2 t+i d \pi / 4}}{t^{d / 2}}+\frac{\operatorname{csch}\left(2 \kappa_{1}\right)\left(r^{2}+4 d \kappa_{3} \operatorname{coth}\left(2 \kappa_{1}\right)-d(d-2) / 4\right)}{2^{d+4} \pi^{\frac{d}{2}}} \frac{e^{i 2 t+i(d+2) \pi / 4}}{t^{1+d / 2}}
\end{aligned}
$$

The third term in the first line of (3.26) comes from the product of the leading $k^{d-1}$ term in expansion of $f$ and $w^{(4)} y^{4} / t$ brought down from the exponential. In the second line we have performed the integral using the general formula $\int_{0}^{\infty} d y y^{d} e^{-y^{2}}=\frac{1}{2} \Gamma\left(\frac{d+1}{2}\right)$ and finally we Wick rotate back to $t \rightarrow-i t$. $I_{2}$ is calculated in a similar manner with the only difference being that in the final answer replace $i \rightarrow-i$ (due to Wick rotation in opposite direction). $I_{1}$ and $I_{2}$ thus combine nicely to give a cosine. Reintroducing the dimensions, the time-dependent part of 2-point function in general is given by

$$
\begin{aligned}
\left\langle\phi\left(\overrightarrow{x_{1}}, t\right) \phi\left(\overrightarrow{x_{2}}, t\right)\right\rangle^{(t d)}= & -\frac{m_{\text {out }}^{d-1} \operatorname{csch}\left(2 \kappa_{1}\right)}{2^{d+1} \pi^{d / 2}} \frac{\cos \left(2 m_{\text {out }} t+d \frac{\pi}{4}\right)}{\left(m_{\text {out }} t\right)^{d / 2}} \\
& +\frac{\operatorname{csch}\left(2 \kappa_{1}\right)\left(r^{2}+4 d \kappa_{3} \operatorname{coth}\left(2 \kappa_{1}\right)-d(d-2) / 4\right)}{2^{d+4} \pi^{\frac{d}{2}}} \frac{\cos \left(2 m_{\text {out }} t+(d+2) \frac{\pi}{4}\right)}{\left(m_{\text {out }} t\right)^{1+d / 2}} \\
& +\mathcal{O}\left(\frac{1}{\left(m_{\text {out }} t\right)^{d / 2+2}}\right)^{(3.27)}
\end{aligned}
$$

The above expression tells us that the leading transient only depends on $\kappa_{1}$. It neither cares about the higher conserved charges nor the separation $r$ which only show at subleading order. We tabulate the leading approach to equilibrium of (2.27) in table 7 . We also tabulate the 2-point function in the ground state (2.17) in table 8. 


\begin{tabular}{|l|c|}
\hline \multicolumn{2}{|c|}{$\left\langle g C C\left|\phi\left(\overrightarrow{x_{1}}, t\right) \phi\left(\overrightarrow{x_{2}}, t\right)\right| g C C\right\rangle$} \\
\hline$d=1$ & $-\frac{\operatorname{csch}\left(2 m_{\text {out }} \kappa_{1}\right)}{4 \sqrt{\pi}} \cos \left(2 m_{\text {out }} t+\frac{\pi}{4}\right) \frac{1}{\sqrt{m_{\text {out }}}}+\mathcal{O}\left(\frac{1}{\left(m_{\text {out }} t\right)^{3 / 2}}\right)$ \\
\hline$d=2$ & $-\frac{m_{\text {out }} \operatorname{csch}\left(2 m_{\text {out }} \kappa_{1}\right)}{8 \pi} \cos \left(2 m_{\text {out }} t+\frac{\pi}{2}\right) \frac{1}{m_{\text {out }} t}+\mathcal{O}\left(\frac{1}{\left(m_{\text {out }} t\right)^{2}}\right)$ \\
\hline$d=3$ & $-\frac{m_{\text {out }}^{2} \operatorname{csch}\left(2 m_{\text {out }} \kappa_{1}\right)}{16 \pi^{3 / 2}} \cos \left(2 m_{\text {out }} t+3 \frac{\pi}{4}\right) \frac{1}{\left(m_{\text {out }} t\right)^{3 / 2}}+\mathcal{O}\left(\frac{1}{\left(m_{\text {out }} t\right)^{5 / 2}}\right)$ \\
\hline$d=4$ & $-\frac{m_{\text {out }}^{3} \operatorname{csch}\left(2 m_{\text {out }} \kappa_{1}\right)}{32 \pi^{2}} \cos \left(2 m_{\text {out }} t+\pi\right) \frac{1}{\left(m_{\text {out }} t\right)^{2}}+\mathcal{O}\left(\frac{1}{\left(m_{\text {out }} t\right)^{3}}\right)$ \\
\hline
\end{tabular}

Table 7. Time-dependent part of 2-point function $\left\langle g C C\left|\phi\left(\overrightarrow{x_{1}}, t\right) \phi\left(\overrightarrow{x_{2}}, t\right)\right| g C C\right\rangle$ in the gCC state defined by $\kappa(k)=\kappa_{1}+\kappa_{3} k^{2}+\kappa_{5} k^{4}+\cdots$. Here $m_{\text {out }}$ is the final mass and $r=\left|\overrightarrow{x_{1}}-\overrightarrow{x_{2}}\right|$.

\begin{tabular}{|l|r|}
\hline \multicolumn{2}{|c|}{$\left\langle 0_{\text {in }}\left|\phi\left(\overrightarrow{x_{1}}, t\right) \phi\left(\overrightarrow{x_{2}}, t\right)\right| 0_{\text {in }}\right\rangle$} \\
\hline$d=1$ & $\frac{\left(m_{\text {out }}^{2}-m^{2}\right)}{8 \sqrt{\pi} m m_{\text {out }}} \cos \left(2 m_{\text {out }} t+\frac{\pi}{4}\right) \frac{1}{\sqrt{m_{\text {out }}}}+\mathcal{O}\left(\frac{1}{\left(m_{\text {out }} t\right)^{3 / 2}}\right)$ \\
\hline$d=2$ & $\frac{\left(m_{\text {out }}^{2}-m^{2}\right)}{16 \pi m} \cos \left(2 m_{\text {out }} t+\frac{\pi}{2}\right) \frac{1}{m_{\text {out }}}+\mathcal{O}\left(\frac{1}{\left(m_{\text {out }} t\right)^{2}}\right)$ \\
\hline$d=3$ & $\frac{\left(m_{\text {out }}^{2}-m^{2}\right) m_{\text {out }}}{32 \pi^{3 / 2} m} \cos \left(2 m_{\text {out }} t+\frac{3 \pi}{4}\right) \frac{1}{\left(m_{\text {out }} t\right)^{3 / 2}}+\mathcal{O}\left(\frac{1}{\left(m_{\text {out }} t\right)^{5 / 2}}\right)$ \\
\hline$d=4$ & $\frac{\left(m_{\text {out }}^{2}-m^{2}\right) m_{\text {out }}^{2}}{64 \pi^{2} m} \cos \left(2 m_{\text {out }} t+\pi\right) \frac{1}{\left(m_{\text {out }} t\right)^{2}}+\mathcal{O}\left(\frac{1}{\left(m_{\text {out }} t\right)^{3}}\right)$ \\
\hline
\end{tabular}

Table 8. Time-dependent part of 2-point function $\left\langle 0_{\text {in }}\left|\phi\left(\overrightarrow{x_{1}}, t\right) \phi\left(\overrightarrow{x_{2}}, t\right)\right| 0_{\text {in }}\right\rangle$ in massive quench. $r=\left|\overrightarrow{x_{1}}-\overrightarrow{x_{2}}\right|$.

\subsection{2 $\partial_{t} \phi \partial_{t} \phi$ and $\partial_{i} \phi \partial_{i} \phi$ correlators}

We also calculate the time-dependent piece of the correlator $\left.\left\langle\partial_{t_{1}} \phi\left(\overrightarrow{x_{1}}, t_{1}\right) \partial_{t_{2}} \phi\left(\overrightarrow{x_{2}}, t_{2}\right)\right\rangle\right|_{t_{1}=t_{2}=t}$ eq. (2.18).

It is a bit surprising that the leading power of $t$ here is also same as before, but it is easy to understand why. For a moment let us rewrite $2 t=t_{1}+t_{2}$. Then take derivatives on $\langle\phi \phi\rangle$ correlator (in $1+1 \mathrm{dim}$. for example) w.r.t. $t_{1}$ and $t_{2}$, and then restore $t_{1}=t_{2}=t$.

$$
\left.\partial_{t_{1}} \partial_{t_{2}}\left[\cos \left(m_{\text {out }}\left(t_{1}+t_{2}\right)+\frac{\pi}{4}\right) \frac{2^{1 / 2}}{\left(t_{1}+t_{2}\right)^{1 / 2}}\right]\right|_{t_{1}=t_{2}=t}=-m_{\text {out }}^{2} \cos \left(2 m_{\text {out }} t+\frac{\pi}{4}\right)\left(\frac{1}{t}\right)^{1 / 2}+\mathcal{O}\left(\frac{1}{t^{3 / 2}}\right)
$$

Clearly the leading power is unchanged when both the derivatives act on the cosine. However the $\partial_{i} \phi 2$-point function in any gCC state, following the same argument as given after eq. (3.24), behaves as

$$
\left\langle\partial_{i} \phi\left(\overrightarrow{x_{1}}, t\right) \partial_{i} \phi\left(\overrightarrow{x_{2}}, t\right)\right\rangle_{t d}=-\frac{d m_{\text {out }}^{d-1} \operatorname{csch}\left(2 m_{\text {out }} \kappa_{1}\right)}{2^{d+2} \pi^{d / 2}} \frac{\cos \left(2 m_{\text {out }} t+(d+2) \frac{\pi}{4}\right)}{\left(m_{\text {out }} t\right)^{d / 2}}+\mathcal{O}\left(\frac{1}{\left(m_{\text {out }} t\right)^{d / 2+1}}\right)
$$

Explicit expressions in various dimensions for these two-point functions for the ground state are tabulated in table 9 . 


\begin{tabular}{|l|l|l|}
\hline & $\left\langle\partial_{t} \phi \partial_{t} \phi\right\rangle_{t d}$ & $\left\langle\partial_{i} \phi \partial_{i} \phi\right\rangle_{t d}$ \\
\hline$d=1$ & $-\frac{m_{\text {out }}\left(m_{\text {out }}^{2}-m^{2}\right) \cos \left(2 m_{\text {out }} t+\frac{\pi}{4}\right)}{8 \sqrt{\pi} m} \frac{1}{\sqrt{m_{\text {out }}}}+\mathcal{O}\left(\frac{1}{\left(m_{\text {out }} t\right)^{3 / 2}}\right)$ & $\frac{m_{\text {out }}\left(m_{\text {out }}^{2}-m^{2}\right) \cos \left(2 m_{\text {out }} t+\frac{3 \pi}{4}\right)}{16 \sqrt{\pi} m} \frac{1}{\left(m_{\text {out }} t\right)^{3 / 2}}+\mathcal{O}\left(\frac{1}{\left(m_{\text {out }} t\right)^{5 / 2}}\right)$ \\
\hline$d=2$ & $-\frac{m_{\text {out }}^{2}\left(m_{\text {out }}^{2}-m^{2}\right) \cos \left(2 m_{\text {out }} t+\frac{\pi}{2}\right)}{16 \pi m} \frac{1}{m_{\text {out }} t}+\mathcal{O}\left(\frac{1}{\left(m_{\text {out }} t\right)^{2}}\right)$ & $\frac{m_{\text {out }}^{2}\left(m_{\text {out }}^{2}-m^{2}\right) \cos \left(2 m_{\text {out }} t+\pi\right)}{16 \pi m} \frac{1}{\left(m_{\text {out }} t\right)^{2}}+\mathcal{O}\left(\frac{1}{\left(m_{\text {out }} t\right)^{3}}\right)$ \\
\hline$d=3$ & $-\frac{m_{\text {out }}^{3}\left(m_{\text {out }}^{2}-m^{2}\right) \cos \left(2 m_{\text {out }} t+\frac{3 \pi}{4}\right)}{32 \pi^{3 / 2} m} \frac{1}{\left(m_{\text {out }} t\right)^{3 / 2}}+\mathcal{O}\left(\frac{1}{\left(m_{\text {out }} t\right)^{5 / 2}}\right)$ & $\frac{3 m_{\text {out }}^{3}\left(m_{\text {out }}^{2}-m^{2}\right) \cos \left(2 m_{\text {out }} t+\frac{5 \pi}{4}\right)}{32 \pi^{3 / 2} m} \frac{1}{\left(m_{\text {out }} t\right)^{5 / 2}}+\mathcal{O}\left(\frac{1}{\left(m_{\text {out }} t\right)^{7 / 2}}\right)$ \\
\hline$d=4$ & $-\frac{m_{\text {out }}^{4}\left(m_{\text {out }}^{2}-m^{2}\right) \cos \left(2 m_{\text {out }} t+\pi\right)}{64 \pi^{2} m} \frac{1}{\left(m_{\text {out }} t\right)^{2}}+\mathcal{O}\left(\frac{1}{\left(m_{\text {out }} t\right)^{3}}\right)$ & $\frac{m_{\text {out }}^{4}\left(m_{\text {out }}^{2}-m^{2}\right) \cos \left(2 m_{\text {out }} t+\frac{3 \pi}{2}\right)}{16 \pi^{2} m} \frac{1}{\left(m_{\text {out }} t\right)^{3}}+\mathcal{O}\left(\frac{1}{\left(m_{\text {out }} t\right)^{4}}\right)$ \\
\hline
\end{tabular}

Table 9. Time-dependent part of 2-point function $\left\langle 0_{\text {in }}\left|\partial_{t} \phi\left(\overrightarrow{x_{1}}, t\right) \partial_{t} \phi\left(\overrightarrow{x_{2}}, t\right)\right| 0_{\text {in }}\right\rangle$ on the left and $\left\langle 0_{\text {in }}\left|\partial_{i} \phi\left(\overrightarrow{x_{1}}, t\right) \partial_{i} \phi\left(\overrightarrow{x_{2}}, t\right)\right| 0_{\text {in }}\right\rangle$ on the right, both for massive quench. Here $r=\left|\overrightarrow{x_{1}}-\overrightarrow{x_{2}}\right|$.

$$
\underset{0}{\stackrel{1 / m}{\bullet} \quad 1 / m_{\text {out }} \quad t \rightarrow} \underset{0}{\bullet} \stackrel{1 / m \quad t \rightarrow \quad 1 / m_{\text {out }} \rightarrow \rightarrow}{\bullet}
$$

(a)

Figure 3. Relevant scales in the theory for the present consideration. Here $r / t$ is considered fixed as the large $t$ limit is taken. In (a) $m_{\text {out }}$ is a finite non-zero quantity. $t \rightarrow \infty$ limit means $m_{\text {out }} t \rightarrow \infty$. This is the case we discuss in the analysis of large time behaviour for quench to non-zero mass. In (b) the limit $m_{\text {out }} \rightarrow 0$ limit is taken first, so that $m_{\text {out }} t \rightarrow 0$; the large $t$ limit in this case can be defined as $m t \rightarrow \infty$. We do not consider this limit in the paper except when $m_{\text {out }}=0$ (this limit is discussed a bit more at the end of the subsection).

\subsection{Some comments on 2-point functions in critical vs. massive quench}

From the results of the previous subsections it seems that the critical correlators behave in a qualitatively different fashion from the those for the massive case, especially in odd spatial dimensions. This seems to indicate an apparent discontinuity as $m_{\text {out }} \rightarrow 0$. This is surprising since the master formula (3.10) for the critical correlator was obtained from that of the massive case (2.17) by putting $m_{\text {out }}=0$ !

Actually there is no true discontinuity at $m_{\text {out }}=0$ in the exact time-dependent correlators. The apparent discontinuity emerges because the limits $m_{\text {out }} \rightarrow 0$ and $t \rightarrow \infty$ do not commute. This can be understood by considering the scales involved in the theory. For any given mass $m_{\text {out }}$, however small, at very large times we will have $t \gg 1 / m_{\text {out }}$ (see figure $3(\mathrm{a})$ ). In other words, the dimensionless quantity $\tilde{t}=m_{\text {out }} t \gg 1$.

However when $m_{\text {out }}=0,1 / m_{\text {out }}=\infty$. No $t$, however large, can exceed $1 / m_{\text {out }}$. Indeed, the limit $m_{\text {out }} \rightarrow 0$ automatically implies $\tilde{t}=m_{\text {out }} t \rightarrow 0$ (see figure $3(\mathrm{~b})$ ).

More mathematically speaking, the two-point functions involve scaling functions of the form $F\left(m t, m_{\text {out }} t\right)$ (omitting the dimensionless variable $r / t$ ). For the massive two-point functions, late times imply that both scaled variables are taken to infinity; in particular $m_{\text {out }} t \rightarrow \infty$. Indeed, the late time massive correlators in the previous section are given in terms of an expansion in $1 /\left(m_{\text {out }} t\right)$; as explained above, this expansion cannot capture the limit $m_{\text {out }} t \rightarrow 0$. Of course, if an exact evaluation of the relevant integral is possible, 
this limit can be taken. ${ }^{12}$ Such a function would then interpolate between the critical and non-critical behaviour. The apparent discontinuity at $m_{\text {out }}=0$ is only an artifact of the large time asymptotic expansion.

\section{The generalized Gibbs ensemble (GGE)}

We have seen above that the time-dependent part of the two-point functions (the part containing the cosine term in (3.1)) vanishes at large times. The remaining term, therefore, represents the asymptotic value of the two-point function. We will now show that the asymptotic value corresponds to that of the two-point function in a generalized Gibbs ensemble (GGE). Before that, we will first recall some relevant facts about a GGE.

The results presented in this section have overlap with earlier literature, as clear from the references cited in the Introduction (see in particular [3] for an early reference). The purpose of this section is to present the results on thermalization to GGE, by the method followed in this paper in a self-contained fashion.

\subsection{GGE}

The generalized Gibbs ensemble (GGE) can be regarded as a certain grand canonical ensemble with an infinite number of chemical potentials $[1,2,8]$ which are appropriate for the description of equilibrium for an integrable model. An integrable model has an infinite number of local commutative conserved charges $\hat{Q}_{i}$. A GGE is described by a density matrix

$$
\rho_{G G E}=\frac{1}{Z_{G G E}} \exp \left[-\sum_{i} \mu_{i} \hat{Q}_{i}\right], Z_{G G E}=\operatorname{Tr} \exp \left[-\sum_{i} \mu_{i} \hat{Q}_{i}\right]
$$

Let us consider a free scalar field $\phi(x, t)$, described by the standard mode expansion

$$
\phi(\vec{k}, t)=a(\vec{k}, t) u(\vec{k}, t)+c c, u(k, t)=\frac{e^{-i \omega(k) t}}{\sqrt{2 \omega(k)}}, \omega(k)=\sqrt{k^{2}+m_{0}^{2}}
$$

and a Hamiltonian $H=\sum_{k} \omega(k) \hat{N}(k)$, where $\hat{N}(k)=a^{\dagger}(k) a(k)$ are the occupation numbers.

This system is clearly integrable; a standard basis of the commuting conserved charges is (cf. (2.7)) $\hat{Q}_{i}=\sum_{k}|k|^{i-1} \hat{N}(k), i=1,2, \ldots$. Another simple basis of charges are the set of occupation numbers $\hat{N}(k)$ themselves. With this choice the GGE is described by the density matrix

$$
\rho_{G G E}=\frac{1}{Z_{G G E}} \exp \left(-\sum_{\vec{k}} \mu(k) \hat{N}(k)\right), Z_{G G E}=\operatorname{Tr} \exp \left(-\sum_{\vec{k}} \mu(k) \hat{N}(k)\right)
$$

The GGE partition is easy to evaluate (most calculations in this subsection are presented in greater detail in appendix D):

$$
Z_{G G E}=\sum_{\{N(k)\}, N(k)=0,1,2, \ldots} \exp \left(-\sum_{\vec{k}} \mu(k) N(k)\right)=\prod_{k}(1-\exp [-\mu(k)])^{-1}
$$

\footnotetext{
${ }^{12}$ Note that in $d=1$ and 2 , there are infrared divergences associated with this limit for $\langle\phi \phi\rangle$ correlators but these problems are not there in correlators of the type $\langle\partial \phi \partial \phi\rangle$.
} 
It is also easy to evaluate the average value of the number operator

$$
\langle\hat{N}(k)\rangle_{G G E}=\operatorname{Tr}\left(\rho_{G G E} \hat{N}(k)\right)=-\frac{\partial}{\partial \mu(k)} \ln Z_{G G E}=\frac{1}{e^{\mu(k)}-1}
$$

With the above ingredients, we can now compute the equal time two-point function in the GGE (for more details and also the case of unequal time correlator, see appendix D):

$$
\begin{aligned}
\left\langle\phi\left(\overrightarrow{x_{1}}, t\right) \phi\left(\overrightarrow{x_{2}}, t\right)\right\rangle_{G G E} & =\operatorname{Tr}\left[\rho_{G G E} \phi\left(\overrightarrow{x_{1}}, t\right) \phi\left(\overrightarrow{x_{2}}, t\right)\right] \\
& =\int \frac{d^{d} k}{(2 \pi)^{d}}|u(\vec{k}, t)|^{2} e^{i \vec{k} \cdot\left(\overrightarrow{x_{1}}-\overrightarrow{x_{2}}\right)}\left[2\langle N(k)\rangle_{G G E}+1\right] \\
& =\int \frac{d^{d} k}{(2 \pi)^{d}} \frac{1}{2 \sqrt{k^{2}+m_{0}^{2}}} e^{i \vec{k} \cdot\left(\overrightarrow{x_{1}}-\overrightarrow{x_{2}}\right)} \operatorname{coth}(\mu(k) / 2)
\end{aligned}
$$

Note that for massless scalars, $H=\sum_{k}|k| a^{\dagger}(k) a(k)=Q_{2}$. Also the total occupation number is always $N=\sum_{k} a^{\dagger}(k) a(k)=Q_{1}$. Hence, we can write (4.2) as

$$
\begin{aligned}
\rho_{G G E} & =\frac{1}{Z_{G G E}} \exp \left[-\left(\mu N+\beta H+\sum_{i=3}^{\infty} \mu_{k} \hat{Q}_{i}\right)\right], Z_{G G E}=\operatorname{Tr} \exp \left[-\left(\mu N+\beta H+\sum_{i=3}^{\infty} \mu_{k} \hat{Q}_{i}\right)\right] \\
\beta & :=\mu_{2}, \mu:=\mu_{1}
\end{aligned}
$$

Thus, a standard thermal (Gibbs) ensemble is a GGE with all $\mu_{i \neq 2}=0$.

\subsection{Equilibration to GGE}

Note that the two-point function in the most general gCC state (2.27) is of the form

$$
\left\langle g C C\left|\phi\left(\vec{x}_{1}, t\right) \phi\left(\vec{x}_{2}, t\right)\right| g C C\right\rangle=\int \frac{d^{d} k}{(2 \pi)^{d}} \frac{e^{i \vec{k} \cdot\left(\overrightarrow{x_{1}}-\overrightarrow{x_{2}}\right)}}{2 \sqrt{k^{2}+m_{\text {out }}^{2}}}[\operatorname{coth}(2 \kappa(k))+\text { time dependent }]
$$

In the preceding sections we have shown that the time-dependent part decays at late times exponentially or by a power law. Hence the gCC two-point function (4.7) asymptotically approaches the GGE two-point function (4.5) provided we identify

$$
m_{0}=m_{\text {out }}, \mu(k)=4 \kappa(k), \text { equivalently } \mu_{i}=4 \kappa_{i}
$$

Indeed, these relations ensure

$$
\langle N(k)\rangle_{G G E}=\langle g C C|N(k)| g C C\rangle
$$

To prove this, note that by identifying $|g C C\rangle$ with $\left|f_{\text {in }}\right\rangle$ and using the top line of (2.20), we get that the right hand side of the above equation is given by $\left(1 / \gamma_{\text {eff }}^{2}-1\right)^{-1}$. By using $\gamma_{\text {eff }}(k)=-\exp [-2 \kappa(k)]$ (see immediately above $(2.22)$ ), the r.h.s. becomes $\left(e^{4 \kappa(k)}-1\right)^{-1}$ which agrees with equation $((4.4))$ with the identification (4.8). Q.E.D.

Thus, we have established the following statement of equilibration:

$$
\left\langle g C C\left|\phi\left(\vec{x}_{1}, t\right) \phi\left(\vec{x}_{2}, t\right)\right| g C C\right\rangle=\left\langle\phi\left(\overrightarrow{x_{1}}, t\right) \phi\left(\overrightarrow{x_{2}}, t\right)\right\rangle_{G G E}+\left(\sim e^{-\gamma t} \text { or } \sim 1 / t^{p}\right)
$$


where the GGE is defined in terms of the gCC by the basic relation (4.9). Since for a free field theory, all correlation functions can be essentially built from the two-point function, the above statement of equilibration is true for all correlators. This constitutes a proof of the GGE version of quantum ergodic hypothesis in the quench models considered in this paper:

$$
\left\langle\psi(t)\left|O\left(x_{1}\right) O\left(x_{2}\right) O\left(x_{3}\right) \ldots\right| \psi(t)\right\rangle \stackrel{t \rightarrow \infty}{\longrightarrow}\left\langle O\left(x_{1}\right) O\left(x_{2}\right) O\left(x_{3}\right)\right\rangle_{G G E}
$$

Eq. (4.8) gives us the important relation between the parameters of the equilibrium ensemble (GGE) with those of the initial state. In the massless case, in terms of the notation of (4.6), we can rewrite (4.8) as

$$
\beta=4 \kappa_{2} ; \quad \mu_{i}=4 \kappa_{i}, i \neq 2
$$

It is clear that the CC state (2.25) equilibrates to a standard Gibbs ensemble with $\mu_{i \neq 2}=0$.

\section{Geometrical interpretation of the correlators in the CC state}

Here we follow [41] to show how the two-point function in a CC state can be identified with an image sum in a slab geometry $R^{d} \times$ interval. This would help us better understand the origin of the difference in thermalization between odd and even dimensions (in short, the odd-even effect). The slab propagator is defined as

$$
\begin{aligned}
G_{\text {slab }}\left(\tau_{1}, \overrightarrow{x_{1}} ; \tau_{2}, \overrightarrow{x_{2}}\right) & =\left\langle\phi\left(\tau_{1}, \overrightarrow{x_{1}}\right) \phi\left(\tau_{2}, \overrightarrow{x_{2}}\right)\right\rangle_{\text {slab }} \\
& =\int \frac{d^{d} k}{(2 \pi)^{d}} e^{i \vec{k} \cdot\left(\overrightarrow{x_{1}}-\overrightarrow{x_{2}}\right)} G_{\text {slab }}\left(\tau_{1}, \tau_{2} ; \vec{k}\right) \\
& =\int \frac{d^{d} k}{(2 \pi)^{d}} \frac{e^{i \vec{k} \cdot\left(\overrightarrow{x_{1}}-\overrightarrow{x_{2}}\right)}}{2|\vec{k}|}\left(S_{t h}\left(\tau_{1}-\tau_{2}\right)+S_{\text {diff }}\left(\tau_{1}+\tau_{2}\right)\right)
\end{aligned}
$$

where

$$
\begin{aligned}
S_{\mathrm{th}}(\tau) & =\sum_{n=0}^{\infty} e^{-|\vec{k}|(2 n L+\tau)}+\sum_{n=1}^{\infty} e^{-|\vec{k}|(2 n L-\tau)} \\
S_{\mathrm{diff}}\left(\tau^{\prime}\right) & =-\sum_{n=0}^{\infty} e^{-|\vec{k}|\left((2 n+1) L+\tau^{\prime}\right)}-\sum_{n=1}^{\infty} e^{-|\vec{k}|\left((2 n-1) L-\tau^{\prime}\right)}
\end{aligned}
$$

The expression (5.1) is obtained by the method of images as shown in the diagram below (see figure 4). The geometry reflects the fact that a CC state of the form $e^{-\kappa_{2} H}|B d\rangle$ can be regarded as a Euclidean time evolution $\Delta \tau=\kappa_{2}$, here denoted by $L / 2$. The suffices $t h$ and diff will be explained below.

It is easy to verify that (5.1) reproduces the two-point function in a CC state. All the four terms in (5.1) can be easily summed to obtain

$$
\begin{aligned}
& S_{\mathrm{th}}\left(\tau_{1}-\tau_{2}\right)+S_{\text {diff }}\left(\tau_{1}+\tau_{2}\right) \\
& \quad=\frac{1}{\left(1-e^{-2|\vec{k}| L}\right)}\left[e^{-|\vec{k}|\left|\tau_{1}-\tau_{2}\right|}+e^{-|\vec{k}|\left(2 L-\left|\tau_{1}-\tau_{2}\right|\right)}-2 e^{-|\vec{k}| L} \cosh \left(|\vec{k}|\left(\tau_{1}+\tau_{2}\right)\right)\right]
\end{aligned}
$$



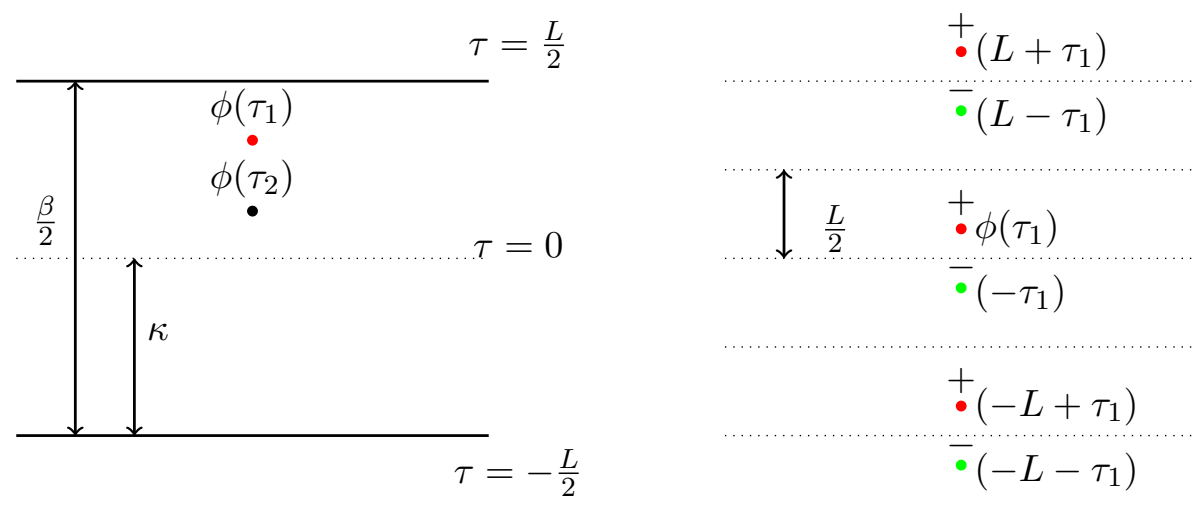

Figure 4. The two-point function between $\phi\left(\tau_{1}\right)$ and $\phi\left(\tau_{2}\right)$ can be regarded as the electrostatic potential between two positive charges of unit magnitude [41]. This follows from the fact that the massless Klein-Gordon equation in Euclidean space is simply the Laplace equation. Here we consider $\phi\left(\tau_{1}\right)$ as a source charge whose electrostatic potential in the slab geometry can be found out by the method of images by regarding the slab as two parallel mirrors. In the right panel we depict by green(red) circles the images obtained by an odd(even) number of reflections of the source. The electrostatic potential in the slab can now be regarded as a sum of that between the probe charge placed at $\tau_{2}$ and each of the images.

Now analytically continuing to real time by $\tau \rightarrow i t$ and identifying $\kappa_{2}=L / 2$ we find the slab propagator is the same as (2.26) with $\kappa_{2}=L / 2$ and all other $\kappa_{i}=0$

$$
\begin{aligned}
& G_{\text {slab }}\left(t_{1}, \overrightarrow{x_{1}} ; t_{2}, \overrightarrow{x_{2}}\right) \\
& \quad=\int \frac{d^{d} k}{(2 \pi)^{d}} \frac{e^{i \vec{k} \cdot\left(\overrightarrow{x_{1}}-\overrightarrow{x_{2}}\right)}}{2|\vec{k}|} \operatorname{csch}\left(2 \kappa_{2}|\vec{k}|\right)\left[\cos \left(|\vec{k}|\left(2 i \kappa_{2}+t_{1}-t_{2}\right)\right)-\cos \left(|\vec{k}|\left(t_{1}+t_{2}\right)\right)\right]
\end{aligned}
$$

This completes the explicit check that the slab propagator (5.1) indeed represents the two-point function in the CC state.

\subsection{Comments on approach to thermalization and the odd-even effect}

The understanding of thermalization from the above geometrical picture goes as follows. First, note that the terms in $S_{\mathrm{th}}$ correspond to Euclidean $R^{d+1} 2$-point function between the test charge with the ' + ' images above and below it. These are precisely the terms which would appear in a cylindrical geometry, which represents a thermal 2-point function with Euclidean time period $\beta=2 L_{2}=4 \kappa_{2}$; hence the suffix ${ }_{t h}$. The terms in $S_{\text {diff }}$, on the other hand, correspond to $R^{d+1}$ 2-point functions between the test charge with the '-' images above and below it; below we will find that these terms vanish at long times, proving that the CC two-point function asymptotically approaches the thermal 2-point function $S_{\mathrm{th}}$, $\beta=4 \kappa_{2}$, which verifies the relation (4.11).

It is clear from the above that $S_{\text {diff }}$ represents the difference between CC correlator and the thermal correlator. For thermalization of the auto-correlator $\left(\vec{x}=\vec{y}=\vec{r}, t_{1} \neq 0\right)$, 
we need to consider the following difference

$$
\begin{aligned}
& G_{C C}\left(\tau_{1}, \vec{r} ; \tau_{2}, \vec{r}\right)-G_{t h}\left(\tau_{1}, \vec{r} ; \tau_{2}, \vec{r}\right)_{\beta}=\frac{\Omega_{d-1}}{2(2 \pi)^{d}} \int_{0}^{\infty} d k k^{d-2} S_{\mathrm{diff}}\left(\tau_{1}+\tau_{2}\right) \\
& =-\frac{\Omega_{d-1}}{2(2 \pi)^{d}} \int_{0}^{\infty} d k k^{d-2}\left(\sum_{n=0}^{\infty} e^{-k((2 n+1) L+\tau)}+\sum_{n=1}^{\infty} e^{-k((2 n-1) L-\tau)}\right) \\
& =-\frac{\Omega_{d-1}(d-2) !}{2(2 \pi)^{d}}\left[\left(\frac{1}{L+\tau}\right)^{d-1}+\sum_{n=1}^{\infty}\left(\frac{1}{(2 n+1) L+\tau}\right)^{d-1}+\sum_{n=1}^{\infty}\left(\frac{1}{(2 n-1) L-\tau}\right)^{d-1}\right]
\end{aligned}
$$

Where $\tau=\tau_{1}+\tau_{2}$. In the last line we have performed the $k$ integral. Note that since $\tau_{1}$ and $\tau_{2}$ lie in the interval $[-L / 2, L / 2]$, allowed values of $\tau$ are in the interval $[-L, L]$.

To see the difference between odd and even $d$, let us choose particular values of $d$.

$\boldsymbol{d}=\mathbf{4}$. We have

$$
\begin{aligned}
& G_{C C}\left(\tau_{1}, \vec{r} ; \tau_{2}, \vec{r}\right)-G_{t h}\left(\tau_{1}, \vec{r} ; \tau_{2}, \vec{r}\right)_{\beta} \\
& =-\frac{1}{8 \pi^{2}}\left[\left(\frac{1}{L+\tau}\right)^{3}+\sum_{n=1}^{\infty}\left(\frac{1}{(2 n+1) L+\tau}\right)^{3}+\sum_{n=1}^{\infty}\left(\frac{1}{(2 n-1) L-\tau}\right)^{3}\right]
\end{aligned}
$$

Now we use the Euler-Maclaurin sum formula for an infintely differentiable function

$$
\sum_{n=1}^{\infty} f(n)=\int_{0}^{\infty} d y f(y)+\frac{f(\infty)-f(0)}{2}+\sum_{k=1}^{\infty} \frac{B_{2 k}}{(2 k) !}\left(f^{(2 k-1)}(\infty)-f^{(2 k-1)}(0)\right)
$$

and apply it to the 2 sums above to get

$$
\begin{aligned}
& S_{+}=\sum_{n=1}^{\infty}\left(\frac{1}{(2 n+1) L+\tau}\right)^{3}=\frac{1}{4 L(L+\tau)^{2}}-\frac{1}{2(L+\tau)^{3}}+\frac{L}{2(L+\tau)^{4}}+\mathcal{O}\left((L+\tau)^{-6}\right) \\
& S_{-}=\sum_{n=1}^{\infty}\left(\frac{1}{(2 n-1) L-\tau}\right)^{3}=\frac{1}{4 L(L+\tau)^{2}}+\frac{1}{2(L+\tau)^{3}}+\frac{L}{2(L+\tau)^{4}}+\mathcal{O}\left((L+\tau)^{-6}\right)
\end{aligned}
$$

and therefore

$$
G_{C C}\left(\tau_{1}, \vec{r} ; \tau_{2}, \vec{r}\right)-G_{t h}\left(\tau_{1}, \vec{r} ; \tau_{2}, \vec{r}\right)_{\beta}=-\frac{1}{8 \pi^{2}}\left(\frac{1}{2 L(L+\tau)^{2}}+\frac{1}{(L+\tau)^{3}}+\frac{L}{(L+\tau)^{4}}\right)+\mathcal{O}\left((L+\tau)^{-6}\right)
$$

Analytically continuing to real time $\left(\tau_{1} \rightarrow i t\right.$ and $\left.\tau_{2} \rightarrow i t\right)$, for $t \rightarrow \infty,{ }^{13}$ we find (after putting $L=2 \kappa_{2}$ )

$$
G_{C C}(t, \vec{r} ; t, \vec{r})-G_{t h}(t, \vec{r} ; t, \vec{r})_{\beta}=\frac{1}{128 \pi^{2} \kappa_{2} t^{2}}+\frac{\kappa_{2}}{128 \pi^{2} t^{4}}+\mathcal{O}\left(t^{-6}\right)
$$

This gives us the power law decay in even dimensions. Also this is precisely the expression for the 2-point function of $\phi$ in the CC state $\left(\kappa_{2 n}=0, n>1\right)$ for $r=0$. We match the

\footnotetext{
${ }^{13}$ Note that although $|\tau| \leq L$, there is no such restriction on $t$ which can be taken to infinity.
} 
Euler-Maclaurin result with the method used before to calculate eq. (3.19) to $\mathcal{O}\left(t^{-20}\right)$ and find exact agreement. Notice that there is a coincident singularity because of $t_{1}=t_{2}$, but that would show up in 'thermal' part $G_{t h}$, so we do not need to worry about it here. Now lets see what happens in odd dimensions.

$\boldsymbol{d}=\mathbf{3}$. Here we have

$$
\begin{aligned}
& G_{C C}\left(\tau_{1}, \vec{r} ; \tau_{2}, \vec{r}\right)-G_{t h}\left(\tau_{1}, \vec{r} ; \tau_{2}, \vec{r}\right)_{\beta} \\
& =-\frac{1}{8 \pi^{2}}\left[\left(\frac{1}{L+\tau}\right)^{2}+\sum_{n=1}^{\infty}\left(\frac{1}{(2 n+1) L+\tau}\right)^{2}+\sum_{n=1}^{\infty}\left(\frac{1}{(2 n-1) L-\tau}\right)^{2}\right]
\end{aligned}
$$

The Euler-Maclaurin sum formula applied here results in

$$
\begin{aligned}
& S_{+}=\sum_{n=1}^{\infty}\left(\frac{1}{(2 n+1) L+\tau}\right)^{2}=\frac{1}{2 L(L+\tau)}-\frac{1}{2(L+\tau)^{2}}+\frac{L}{3(L+\tau)^{3}}+\mathcal{O}\left((L+\tau)^{-5}\right) \\
& S_{-}=\sum_{n=1}^{\infty}\left(\frac{1}{(2 n-1) L-\tau}\right)^{2}=-\frac{1}{2 L(L+\tau)}-\frac{1}{2(L+\tau)^{2}}-\frac{L}{3(L+\tau)^{3}}+\mathcal{O}\left((L+\tau)^{-5}\right)
\end{aligned}
$$

Together with the $n=0$ term

$$
G_{C C}\left(\tau_{1}, \vec{r} ; \tau_{2}, \vec{r}\right)-G_{t h}\left(\tau_{1}, \vec{r} ; \tau_{2}, \vec{r}\right)_{\beta}=-\frac{1}{8 \pi^{2}}\left(\frac{1}{(L+\tau)^{2}}+S_{+}+S_{-}\right)=0
$$

We expect the vanishing result found above to continue to all orders in the $1 / t$ expansion (we have explicitly checked this fact till $\mathcal{O}\left(t^{-20}\right)$. Indeed this had to be the case because we know the answer goes as $e^{-\pi t / \kappa_{2}}$ (see (C.8)) which does not have a Taylor expansion in $1 / t$ around 0 .

It is not difficult to generalize the above results to arbitrary dimensions $d>2$ for example by using the recursion relations (see appendix B). The summary is that

$$
G_{C C}\left(t_{1}, \vec{r} ; t_{2}, \vec{r}\right)-G_{t h}\left(t_{1}, \vec{r} ; t_{2}, \vec{r}\right)_{\beta} \sim \begin{cases}t^{-(d-2)} & \mathrm{d} \text { is even } \\ e^{-\pi t / \kappa_{2}} & \mathrm{~d} \text { is odd }\end{cases}
$$

There is a pictorial way to understand the decay of the time-dependent part of the CC correlator. In the slab diagram imagine the analytic continuation of time $\left(\tau_{j} \rightarrow \tau_{j}+i t_{j}\right)$ with real time axis perpendicular to the plane of the paper (see figure 5). The spatial dimensions are all in the horizontal direction. Note that upon analytic continuation to real time, the 'even' and 'odd' images move in opposite directions. Also notice that $S_{t h}$ involves the correlator between the probe(black) and the even images of the source (marked red); the way to see this is to note that such correlators all involve the time difference $t_{1}-t_{2}$ as expected from (5.1). Similarly while $S_{\text {diff }}$ involves the correlator between the probe(black) and the odd images of the source (marked green); the way to see this is to note that such correlators all involve the time difference $t_{1}+t_{2}$ again as expected from (5.1). When $t_{1}$ and $t_{2}$ are increased by the same amount, the Euclidean separations involved in $S_{t h}$ remain the 
$\cdot\left(2 L-\tau_{1}\right)$
$\cdot\left(L+\tau_{1}\right)$

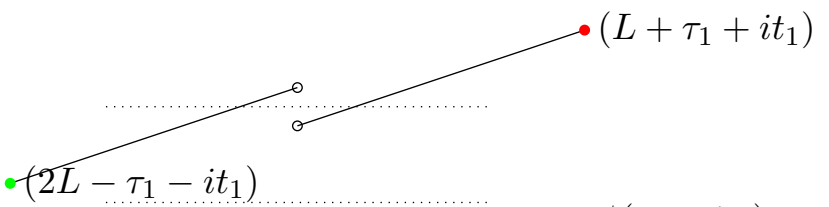

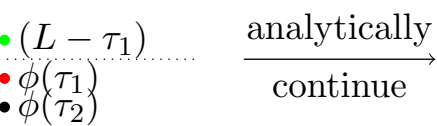

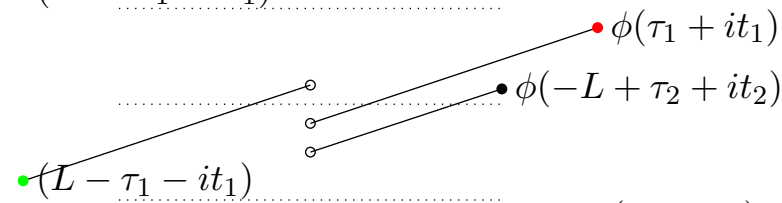

$\cdot\left(-\tau_{1}\right)$
$\cdot\left(-L+\tau_{1}\right)$

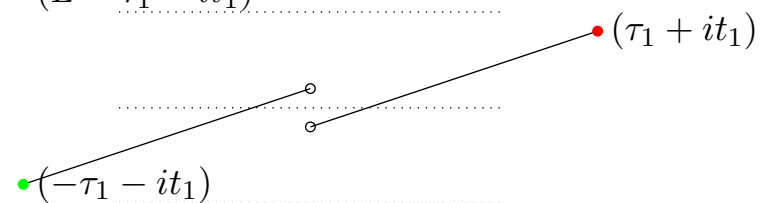

Figure 5. In the above diagram we consider $\phi\left(\tau_{1}\right)$ as the source and $\phi\left(\tau_{2}\right)$ as a probe. As we analytically continue $\tau_{j} \rightarrow \tau_{j}+i t_{j}$, and $t_{1}$ and $t_{2}$ are increased by the same amount, the red images (even reflections of the source) move in the same direction as the probe, while the green images (odd reflections) move in the opposite direction. For large $t_{1}$ and $t_{2}$ with $t_{1}-t_{2}$ held fixed, the probe $\phi\left(\tau_{2}+i t_{2}\right)$ sees the green images at progressively farther distances and hence their correlators decay. Thus in this limit the slab correlator approaches the thermal correlator.

same whereas they go to infinity in case of $S_{\text {diff }}$. This is the reason why $S_{\text {diff }}$ decays in time in this limit and the slab correlator approaches the thermal value. ${ }^{14}$

In the above we have considered separation between the source and the probe only in time but the entire argument goes through for fixed, finite spatial separation $r$ as we take $t \rightarrow \infty$

\subsection{The thermal auto-correlator}

In the previous subsection we showed how the difference between the $\mathrm{CC}$ correlator and the thermal auto-correlator vanishes as $t_{1}, t_{2} \rightarrow \infty$. In this section we will compute the thermal auto-correlator itself. We will find that this too shows an odd-even difference between dimensions as one takes the limit of large $t \equiv t_{1}-t_{2}$.

The quantity to compute is

$$
\begin{aligned}
G_{t h}\left(\tau_{1}-\tau_{2}=\tau ; r=0\right)_{\beta} & =\frac{\Omega_{d-1}}{2(2 \pi)^{d}} \int_{0}^{\infty} d k k^{d-2}\left[\sum_{n=0}^{\infty} e^{-k(n \beta+\tau)}+\sum_{n=1}^{\infty} e^{-k(n \beta-\tau)}\right] \\
& =\frac{\Omega_{d-1}(d-2) !}{2(2 \pi)^{d}}\left[\sum_{n=0}^{\infty}\left(\frac{1}{n \beta+\tau}\right)^{d-1}+\sum_{n=1}^{\infty}\left(\frac{1}{n \beta-\tau}\right)^{d-1}\right]
\end{aligned}
$$

Note that here $\tau=\tau_{1}+\tau_{2}$. As in the previous subsection lets analyze this in $d=4$ and $d=3$ separately.

$\boldsymbol{d}=$ 4. Here

$$
G_{t h}\left(\tau_{1}-\tau_{2}=\tau ; r=0\right)_{\beta}=\frac{1}{8 \pi^{2}}\left[\frac{1}{\tau^{3}}+\sum_{n=1}^{\infty}\left(\frac{1}{n \beta+\tau}\right)^{3}+\sum_{n=1}^{\infty}\left(\frac{1}{n \beta-\tau}\right)^{3}\right]
$$

\footnotetext{
${ }^{14}$ We thank Abhijit Gadde for suggesting this.
} 
Applying the Euler-Maclaurin formula

$$
\begin{aligned}
& S_{+}=\sum_{n=1}^{\infty}\left(\frac{1}{n \beta+\tau}\right)^{3}=\frac{1}{2 \beta \tau^{2}}-\frac{1}{2 \tau^{3}}+\frac{\beta}{4 \tau^{4}}+\mathcal{O}\left(\tau^{-6}\right) \\
& S_{-}=\sum_{n=1}^{\infty}\left(\frac{1}{n \beta-\tau}\right)^{3}=\frac{1}{2 \beta \tau^{2}}+\frac{1}{2 \tau^{3}}+\frac{\beta}{4 \tau^{4}}+\mathcal{O}\left(\tau^{-6}\right)
\end{aligned}
$$

After analytically continuing $\tau=\tau_{1}+\tau_{2} \rightarrow i t_{1}+i t_{2}=i t$, together with the $n=0$ term, for large $t$

$$
G_{t h}\left(\tau_{1}-\tau_{2}=\tau ; r=0\right)=\frac{i}{8 \pi^{2} t^{3}}-\frac{1}{8 \pi^{2} \beta t^{2}}+\frac{\beta}{16 \pi^{2} t^{4}}+\mathcal{O}\left(t^{-6}\right)
$$

This matches the precisely the answer (C.22), obtained from the integral formula (D.3).

$\boldsymbol{d}=3$. Here

$$
G_{t h}\left(\tau_{1}-\tau_{2}=\tau ; r=0\right)_{\beta}=\frac{1}{8 \pi^{2}}\left[\frac{1}{\tau^{2}}+\sum_{n=1}^{\infty}\left(\frac{1}{n \beta+\tau}\right)^{2}+\sum_{n=1}^{\infty}\left(\frac{1}{n \beta-\tau}\right)^{2}\right]
$$

Applying the Euler-Maclaurin formula

$$
\begin{aligned}
& S_{+}=\sum_{n=1}^{\infty}\left(\frac{1}{n \beta+\tau}\right)^{2}=\frac{1}{\beta \tau}-\frac{1}{2 \tau^{2}}+\frac{\beta}{6 \tau^{3}}+\mathcal{O}\left(\tau^{-5}\right) \\
& S_{-}=\sum_{n=1}^{\infty}\left(\frac{1}{n \beta-\tau}\right)^{2}=-\frac{1}{\beta \tau}-\frac{1}{2 \tau^{2}}-\frac{\beta}{6 \tau^{3}}+\mathcal{O}\left(\tau^{-5}\right)
\end{aligned}
$$

After analytically continuing $\tau=\tau_{1}+\tau_{2} \rightarrow i t_{1}+i t_{2}=i t$, for large $t$, together with the $n=0$ term

$$
G_{t h}\left(\tau_{1}-\tau_{2}=\tau ; r=0\right)_{\beta}=\frac{1}{8 \pi^{2}}\left(\frac{1}{\tau^{2}}+S_{+}+S_{-}\right)=0 \text { up to } \mathcal{O}\left(t^{-5}\right)
$$

In fact, it turns out that the Taylor coefficients vanish to all orders. This is consistent with the behaviour at $d=3, G_{t h} \sim e^{-\gamma t}$ which is independently obtained in appendix C.2 see equation (C.16). Note that $e^{-\gamma t}$ has an essential singularity at $1 / t=0$ and hence does not have a Taylor expansion in terms of $1 / t$.

\section{$6 \quad$ Kaluza-Klein interpretation of thermal correlators}

In this section, we will study a possible odd-even effect in thermal correlators from the Euclidean viewpoint by considering the time circle (of radius $\beta=1 / T$ ) as a Kaluza-Klein direction.

In the previous section we considered the time-dependence of thermal correlators, that is, thermal two-point functions with no spatial separation. In this section, we will look at thermal 2-point function at space-like separation. A convenient way to look at this is 
the Kaluza-Klein reduction. Demanding periodicity in Euclidean time, the normal mode expansion of a scalar field in $R^{d} \times S^{1}$ is given by

$$
\phi(\tau, \vec{r})=\sum_{n} \phi_{n}(\vec{r}) e^{i 2 \pi n \tau / \beta}
$$

In case the post-quench theory is critical, $\phi$ obeys massless Klein-Gordon equation in $d+1$ dimensions $\partial^{\mu} \partial_{\mu} \phi(\tau, \vec{r})=0$. In terms of the modes one gets

$$
\left(\partial^{i} \partial_{i}-\frac{4 \pi^{2} n^{2}}{\beta^{2}}\right) \phi_{n}(\vec{r})=0
$$

which is again a Klein-Gordon equation in one less dimension but now the fields $\phi_{n}(\vec{r})$ acquire a (thermal) mass $m_{n}=\frac{4 \pi^{2} n^{2}}{\beta^{2}}$. The equal-time thermal 2-point function in terms of the modes is

$$
\begin{aligned}
\langle\phi(0, \vec{r}) \phi(0, \overrightarrow{0})\rangle_{\beta} & =\frac{1}{\beta} \sum_{n}\left\langle\phi_{n}(\vec{r}) \phi_{n}(\overrightarrow{0})\right\rangle_{\beta} \\
& =\frac{1}{\beta} \sum_{n} \int \frac{d^{d} k}{(2 \pi)^{d}} \frac{e^{i k r \cos \theta}}{k^{2}+m_{n}^{2}}
\end{aligned}
$$

This can be evaluated exactly

$$
\langle\phi(0, \vec{r}) \phi(0, \overrightarrow{0})\rangle_{\beta}=\frac{a_{d}}{r^{d-2}}+2 a_{d} \sum_{n=1}^{\infty} \frac{e^{-2 \pi n r / \beta}}{r^{d-2}}
$$

where $a_{d}$ is a dimension-dependent constant. In $d=2, r^{2-d}$ is replaced by $\log r$. In all dimensions we get a Yukawa like exponential decay for the non-zero modes. But in the $r \rightarrow \infty$ limit or equivalently the $\beta \rightarrow 0$ limit, only the power law term, corresponding to the $n=0$ survives. This happens in all dimensions even or odd. This is expected because in the strict $\beta \rightarrow 0$, the circumference of the cylinder shrinks so that the insertions of the operators do not see the cylinder. Note that the surviving power law term corresponds to the Green's function for the dimensionally reduced Euclidean geometry $R^{d}$.

Note the appearance of a power law behaviour at all dimensions in contrast with the thermal auto-correlator in the previous section, which has an exponential/power law behaviour depending on whether the spatial dimension $d$ is odd/even.

\section{Discussion}

In this paper, we presented a self-contained discussion of thermalization of local correlators in free scalar field theories. One of the main results was the difference in relaxation to thermalization depending on odd or even dimensions, are already summarised in the introduction.

It would be interesting to see if the dimension-dependence of relaxation to equilibrium is true in other free field theories such as theories of fermions or electrodynamics, in more 
general integrable models, or in more general, interacting theories. Progress in this direction can be made by considering $O(N)$ models, involving an $N$-tuplet of scalar fields, $\phi_{i}$, $i=1,2, \ldots, N$ which is defined by an action

$$
S=-\int d^{d+1} x\left[\partial_{\mu} \phi_{i}(x) \partial_{\mu} \phi_{i}(x)-m^{2} \phi_{i} \phi_{i}-\frac{\lambda}{4 !}\left(\phi_{i} \phi_{i}\right)^{2}\right]
$$

The model becomes tractable in the large $N$ limit: $N \rightarrow \infty, \lambda N=$ fixed. Quantum quench in such models have been discussed in some detail in dimensions $d=1,2,3$ in [41] where the mass and the coupling constant makes a sudden transition in values $\left(m_{0}, \lambda_{0}\right) \rightarrow(m, \lambda)$ at $t=0$, where $m_{0}$ and $m$ represent renormalized masses before and after the quench. A main result of [41] is that the post-quench phase can be described by an effective mass parameter $m_{\mathrm{eff}}(t)$ which settles down to a value $m_{*}$ fast enough that the quench in the $O(N)$ model can be approximated by a sudden quench in a free theory from renormalized mass $m_{0}$ to $m_{*}$. All information about the coupling constants $\lambda_{0}$ and $\lambda$ are encoded, in the large $N$ limit, in the formula for $m_{*}$, given by

$$
\begin{aligned}
m_{*}^{2} & =m^{2}+\frac{\lambda}{2} \frac{\Omega_{d}}{(2 \pi)^{d}}\left[m_{0}^{d-1} f_{d}\left(\frac{m_{*}}{m_{0}}\right)+m_{*}^{d-1} h_{d}\left(\frac{m}{m_{*}}\right)\right] \\
f_{d}(s) & =\int_{0}^{\infty} d k k^{d-1} \frac{\left(\sqrt{k^{2}+1}-\sqrt{k^{2}+s^{2}}\right)^{2}}{4 \sqrt{k^{2}+1}\left(k^{2}+s^{2}\right)} \\
h_{d}(s) & =\int_{0}^{\Lambda \rightarrow \infty} d k k^{d-1}\left(\frac{1}{k^{2}+1}-\frac{1}{k^{2}+1}\right)
\end{aligned}
$$

It is found that

- In $d=1$, the $m=0$ implies that $m_{*}=0$ (see [41]). Hence one excepts that the exponential relaxation $e^{-\gamma t}$ discussed in our paper, persists even with $O(N)$ interactions in the large $N$ limit. $^{15}$

- In $d=2$ and $d=3$, with $m=0$ but $\lambda \neq 0$, one has $m_{*} \neq 0$. Hence the massless quench, in the presence of interaction, becomes a massive one and the relaxation to thermal equilibrium changes accordingly (in particular, in $d=3$, an exponential relaxation changes to a (modulated) power law relaxation). One can ask the question, if there some choice of $m, \lambda$ for which $m_{*}=0$ ? The answer seems to be yes for negative values of $m^{2} .{ }^{16}$ Hence exponential relaxation to thermalization is still possible, in $d=3$, under such circumstance (as against $d=2$ where it will be power law). Thus, the odd-even effect can still persist under such circumstance. Details of these arguments are being worked out in [46].

\footnotetext{
${ }^{15}$ We are assuming here that the time-dependent effective mass settles down to $m_{*}$ at a rate faster than the exponential thermalization of correlators, so that one can use the italicized result in the previous paragraph.

${ }^{16}$ Note that this is similar to the fact that in $O(N)$ models the critical surface (renormalized mass $=0$ ) is given by negative bare mass-squared. We would like to thank Ganpathy Murthy for a useful discussion on this point.
} 
Another remark that we would like to make is that the odd-even effect is strongly reminiscent of the behaviour of retarded propagators for massless particles, which have support only on the forward light cone in odd spatial dimensions, and inside the forward light cone in even spatial dimensions. The 'leaking of the propagator' inside the light cone in even space dimensions has been termed a violation of the strong Huygen's principle (see, e.g. $[47,48]$ for some physical realization of this phenomenon), since Huygen's construction of a propagating light front is based on propagation along the light cone. We found that there are mathematical similarities between this peculiarity of the retarded propagator and the odd-even effect described in this paper (both are due to difference in the integration over angles in odd vs even dimensions), although we were not able to derive one as a consequence of the other. ${ }^{17}$

The power law approach to thermalization does not appear to have a holographic counterpart; e.g. quasinormal decay of black hole perturbations has an exponential form. In the context of an $\mathrm{O}(\mathrm{N})$ model in $2+1$ dimensions, the above discussion would seem to suggest a power law decay even in the interacting model. It would be interesting to explore the holographic dual in this context.

\section{Acknowledgments}

We would like to thank Alexander Abanov, Sumit Das, Avinash Dhar, Fabian Essler, Abhijit Gadde, Rajesh Gopakumar, R. Loganayagam, Juan Maldacena, Shiraz Minwalla, Ganpathy Murthy, Harvey Reall, Joseph Samuel and Spenta Wadia for discussions. P.B. would like to thank the Department of Theoretical Physics, TIFR, for the research opportunity during the final year of her M.Sc and the Infosys Foundation for financial support. G.M. would like to thank the International Centre for Theoretical Sciences, Bangalore for the opportunity to present/discuss various stages of this work during various meetings and seminars over the past year. We would also like to thank the anonymous referee for suggesting many improvements in the presentation of the paper and bringing to our attention a large number of references. This work was supported in part by the Infosys foundation for the study of quantum structure of spacetime.

\section{A Dirichlet boundary state and relation to post-quench state}

A Dirichlet boundary state is defined by

$$
\phi(x)|D\rangle=0
$$

In terms of the mode expansion (at $t=0$ )

$$
\phi(x)|D\rangle=\int \frac{d^{d} k}{(2 \pi)^{d}} e^{\iota \vec{k} \cdot \vec{x}} \phi(\vec{k})|D\rangle=\int \frac{d^{d} k}{(2 \pi)^{d}} \frac{e^{i \vec{k} \cdot \vec{x}}}{\sqrt{2 \omega_{k}}}\left(a(\vec{k})+a^{\dagger}(-\overrightarrow{-k})\right)|D\rangle
$$

\footnotetext{
${ }^{17}$ We thank R. Loganayagam, H. Reall and J. Samuel for illuminating discussions on this issue.
} 
Clearly this is zero if $\forall \vec{k}$ the following equation is satisfied

$$
\left[a(\vec{k})+a^{\dagger}(\overrightarrow{-k})\right]|D\rangle=\left[\frac{\partial}{\partial a^{\dagger}(\vec{k})}+a^{\dagger}(\overrightarrow{-k})\right]|D\rangle=0
$$

One can check that

$$
|D\rangle=\exp \left[-\frac{1}{2} \int \frac{d^{d} k}{(2 \pi)^{d}} a^{\dagger}(\vec{k}) a^{\dagger}(\overrightarrow{-k})\right]\left|0_{\text {out }}\right\rangle
$$

easily satisfies the above equation. One can write the in ground state as (through a Bogoliubov transformation)

$$
\left|0_{\text {in }}\right\rangle=\exp \left[\frac{1}{2} \int \frac{d^{d} k}{(2 \pi)^{d}} \gamma(k) a_{\text {out }}^{\dagger}(\vec{k}) a_{\text {out }}^{\dagger}(\overrightarrow{-k})\right]\left|0_{\text {out }}\right\rangle
$$

As we shown in [35] one can write

$$
\left|0_{\text {in }}\right\rangle=\exp \left[-\frac{1}{2} \int \frac{d^{d} k}{(2 \pi)^{d}} \kappa(k) a_{\text {out }}^{\dagger}(\vec{k}) a_{\text {out }}^{\dagger}(\overrightarrow{-k})\right]|D\rangle
$$

where $\kappa(k)$ is related to $\gamma(k)$ through equation (2.5).

\section{B Recursion relation}

Here we derive the recursive differential operator for the for the most general gCC correlator. Starting from the 2 point function of $\phi$ fields in $d+1$ dimensions $\left(\omega_{\text {out }}=\sqrt{k^{2}+m_{\text {out }}^{2}}\right)$

$$
\begin{aligned}
& \left\langle\phi\left(x_{1}, t_{1}\right) \phi\left(x_{2}, t_{2}\right)\right\rangle_{g C C}^{(d)} \\
& =\int \frac{d^{d} k}{(2 \pi)^{d}} \frac{e^{i \vec{k} \cdot\left(\overrightarrow{x_{1}}-\overrightarrow{x_{2}}\right)}}{2 \omega_{\text {out }}} \operatorname{csch}(2 \kappa(k))\left[\cos \left(\omega_{\text {out }}\left(t_{1}-t_{2}\right)+2 i \kappa(k)\right)-\cos \left(\omega_{\text {out }}\left(t_{1}+t_{2}\right)\right)\right] \\
& =\int \frac{d^{d} k}{(2 \pi)^{d}} \frac{e^{i \vec{k} \cdot\left(\overrightarrow{x_{1}}-\overrightarrow{x_{2}}\right)}}{2 \omega_{\text {out }}} \operatorname{csch}(2 \kappa(k))\left[\cos \left(\omega_{\text {out }} t_{-}+2 i \kappa(k)\right)-\cos \left(\omega_{\text {out }} t_{+}\right)\right]
\end{aligned}
$$

Here $t_{-}=t_{1}-t_{2}, t_{+}=t_{1}+t_{2}$ and $\kappa(k)=\sum_{n} \kappa_{2 n}|\vec{k}|^{2 n-1}$ represents the most general gCC state (see eq. (2.22)). We now connect this to the correlator in $(d-2)+1$ dimensions. One can write

$$
\begin{aligned}
& \left\langle\phi\left(x_{1}, t_{1}\right) \phi\left(x_{2}, t_{2}\right)\right\rangle_{g C C}^{(d)} \\
& =\frac{\Omega_{d-2}}{2(2 \pi)^{d}} \int_{0}^{\infty} d k \frac{k^{d-1}}{\omega_{\text {out }}} \int_{0}^{\pi} \mathrm{d} \theta e^{i k r \cos \theta}(\sin \theta)^{d-2}\left[\cos \left(2 i \kappa(k)+\omega_{\text {out }} t_{-}\right)-\cos \left(\omega_{\text {out }} t_{+}\right)\right] \\
& =\frac{\Omega_{d-2}}{2(2 \pi)^{d}} \int_{0}^{\infty} d k \frac{k^{d-3}}{\omega_{\text {out }}} \int_{0}^{\pi} \mathrm{d} \theta e^{i k r \cos \theta}(\sin \theta)^{d-4}\left[\cos \left(2 i \kappa(k)+\omega_{\text {out }} t_{-}\right)-\cos \left(\omega_{\text {out }} t_{+}\right)\right] \times k^{2} \sin ^{2} \theta \\
& =\frac{\Omega_{d-2}}{2(2 \pi)^{d}} \int_{0}^{\infty} d k \frac{k^{d-3}}{\omega_{\text {out }}} \int_{0}^{\pi} \mathrm{d} \theta e^{i k r \cos \theta}(\sin \theta)^{d-4}\left[\cos \left(2 i \kappa(k)+\omega_{\text {out }} t_{-}\right)-\cos \left(\omega_{\text {out }} t_{+}\right)\right] \times\left(\omega_{\text {out }}^{2}-m_{\text {out }}^{2}-k^{2} \cos ^{2} \theta\right) \\
& =\frac{\Omega_{d-2}}{2(2 \pi)^{d}}\left(-\partial_{t_{-}}^{2}-\partial_{t_{+}}^{2}+m_{\text {out }}^{2}+\partial_{r}^{2}\right) \int_{0}^{\infty} d k \frac{k^{d-3}}{\omega_{\text {out }}} \int_{0}^{\pi} \mathrm{d} \theta e^{i k r \cos \theta}(\sin \theta)^{d-4}\left[\cos \left(2 i \kappa(k)+\omega_{\text {out }} t_{-}\right)-\cos \left(\omega_{\text {out }} t_{+}\right)\right] \\
& =\frac{\Omega_{d-2}}{4 \pi^{2} \Omega_{d-4}}\left(-\partial_{t_{-}}^{2}-\partial_{t_{+}}^{2}+m_{\text {out }}^{2}+\partial_{r}^{2}\right) \frac{\Omega_{d-4}}{2(2 \pi)^{d-2}} \int_{0}^{\infty} d k \frac{k^{d-3}}{\omega_{\text {out }}} \int_{0}^{\pi} d \theta e^{i k r \cos \theta}(\sin \theta)^{d-4} \\
& \times\left[\cos \left(2 i \kappa(k)+\omega_{\text {out }} t_{-}\right)-\cos \left(\omega_{\text {out }} t_{+}\right)\right] \\
& =\frac{\Omega_{d-2}}{4 \pi^{2} \Omega_{d-4}}\left(-\partial_{t_{-}}^{2}-\partial_{t_{+}}^{2}+m_{\mathrm{out}}^{2}+\partial_{r}^{2}\right)\left\langle\phi\left(x_{1}, t_{1}\right) \phi\left(x_{2}, t_{2}\right)\right\rangle_{g C C}^{(d-2)}
\end{aligned}
$$


$\Omega_{d-1}$ is the solid angle of a $d-1$ dimensional spherical surface in $d$ spatial dimensions. In the third line we have used $k^{2}=\omega_{\text {out }}^{2}-m_{\text {out }}^{2}$. The main point being used here is the fact that one can obtain the extra $k^{2} \sin (\theta)^{2}$ by acting with the derivatives. Also notice that $t_{-}$and $t_{+}$act as independent variables. This derivation also holds for the ground state quench as can be verified directly from equation (2.16).

\section{Details of critical quench calculations}

\section{C.1 2+1 dimensions}

The details of calculation of correlators in $d=2$ are presented here.

\section{C.1.1 CC state}

The time-dependent piece of the 2-point function of $\partial_{t} \phi$ in arbitrary gCC state is

$$
\left\langle\partial_{t} \phi\left(\overrightarrow{x_{1}}, t\right) \partial_{t} \phi\left(\overrightarrow{x_{2}}, t\right)\right\rangle_{t d}=\int_{-\infty}^{\infty} \frac{d^{d} k}{2(2 \pi)^{d}} e^{i \vec{k} \cdot\left(\overrightarrow{x_{1}}-\overrightarrow{x_{2}}\right)} \sqrt{k^{2}+m_{\text {out }}^{2}} \cos \left(2 t \sqrt{k^{2}+m_{\text {out }}^{2}}\right) \operatorname{csch}(2 \kappa(k))
$$

which in $2+1$ for critical quench is

$$
\begin{aligned}
& \left\langle\partial_{t} \phi\left(\overrightarrow{x_{1}, t}\right) \partial_{t} \phi\left(\overrightarrow{x_{2}}, t\right)\right\rangle_{t d} \\
& \quad=\frac{1}{4 \pi} \int_{0}^{\infty} d k e^{i k r} k^{2} J_{0}(k r) \cos (2 k t) \operatorname{csch}(2 \kappa(k)) \\
& \quad=\frac{1}{4 \pi^{2}} \int_{0}^{\pi} d \phi \int_{0}^{\infty} d k e^{i k r} k^{2} \cos (k r \sin \phi) \cos (2 k t) \operatorname{csch}(2 \kappa(k)) \\
& \quad=\frac{1}{16 \pi^{2}} \int_{0}^{\pi} d \phi \int_{0}^{\infty} d k e^{i k r} k^{2} \operatorname{csch}(2 \kappa(k))\left(e^{i k a p}+e^{i k a m}+e^{-i k a p}+e^{-i k a m}\right)
\end{aligned}
$$

where we have exponentiated the cosine in terms of $a p=2 t+r \sin \phi$ and $a m=2 t-r \sin \phi$. For $\kappa(k)=\kappa_{2} k$, these integrals are easily performed in Mathematica in terms of the Polygamma function

$$
\int_{0}^{\infty} d k e^{i k r} k^{2} \operatorname{csch}\left(2 \kappa_{2} k\right) e^{i k a p}=-\frac{\psi^{(2)}\left(\frac{1}{2}-\frac{i a p}{4 \kappa_{2}}\right)}{512 \pi^{2} \kappa_{2}^{3}}
$$

After performing the $k$ integral, we expand the polygammas for large $t$, and then perform the $\phi$ integral to get

$$
\left\langle\partial_{t} \phi\left(\overrightarrow{x_{1}}, t\right) \partial_{t} \phi\left(\overrightarrow{x_{2}}, t\right)\right\rangle_{t d}=-\frac{1}{32 \pi \kappa_{2} t^{2}}-\frac{8 \kappa_{2}^{2}+3 r^{2}}{256 \pi \kappa_{2} t^{4}}+\mathcal{O}\left(t^{-6}\right)
$$

\section{C.1.2 Ground state}

The time-dependent piece of 2-point function of $\partial_{t} \phi$ in the ground state is (2.18)

$$
\left\langle 0_{\text {in }}\left|\partial_{t} \phi\left(\overrightarrow{x_{1}}, t\right) \partial_{t} \phi\left(\overrightarrow{x_{2}}, t\right)\right| 0_{\text {in }}\right\rangle_{t d}=-\int \frac{d^{d} k}{(2 \pi)^{d}} \frac{\left(m_{\text {out }}^{2}-m^{2}\right)}{4 \sqrt{k^{2}+m^{2}}} e^{i \vec{k} \cdot\left(\overrightarrow{x_{1}}-\overrightarrow{x_{2}}\right)} \cos \left(2 t \sqrt{k^{2}+m_{\text {out }}^{2}}\right)
$$


which in $2+1 \mathrm{~d}$ for critical quench is

$$
\begin{aligned}
& \left\langle 0_{\text {in }}\left|\partial_{t} \phi\left(\overrightarrow{x_{1}}, t\right) \partial_{t} \phi\left(\overrightarrow{x_{2}}, t\right)\right| 0_{\text {in }}\right\rangle_{t d} \\
& =\int_{0}^{\infty} d k \frac{k J_{0}(k r)}{8 \pi \sqrt{k^{2}+m^{2}}} m^{2} \cos (2 k t) \\
& =\frac{m^{2}}{8 \pi^{2}} \int_{0}^{\pi} d \phi \int_{0}^{\infty} d k \frac{k}{\sqrt{k^{2}+m^{2}}} \cos (2 k t) \cos (k r \sin \phi) \\
& =\frac{m^{2}}{32 \pi^{2}} \int_{0}^{\pi} d \phi \int_{0}^{\infty} d k \frac{k}{\sqrt{k^{2}+m^{2}}}\left(e^{i k a p}+e^{i k a m}+e^{-i k a p}+e^{-i k a m}\right)
\end{aligned}
$$

Naively doing the integral, we find that $\int_{0}^{\infty} \frac{m^{2} k e^{i k a}}{32 \pi^{2} \sqrt{k^{2}+m^{2}}}$ diverges because as $k \rightarrow \infty$, the integrand is just $e^{i k a}$. We regulate this by $k \rightarrow k(1 \pm i \epsilon)$, performing the $\mathrm{k}$ integral (alternatively Laplace transform with respect to $\epsilon$ ) and then taking $\epsilon \rightarrow 0$. The final result is $\left\langle 0_{\text {in }}\left|\partial_{t} \phi\left(\overrightarrow{x_{1}}, t\right) \partial_{t} \phi\left(\overrightarrow{x_{2}}, t\right)\right| 0_{\text {in }}\right\rangle_{t d}=\int_{0}^{\pi} d \phi \frac{m^{3}}{32 \pi}\left(-\boldsymbol{L}_{-1}(\operatorname{amm})+I_{1}(\operatorname{amm})-\boldsymbol{L}_{-1}(\operatorname{ap} m)+I_{1}(\operatorname{ap} m)\right)$

Now using the fact that modified Struve function has the asymptotic form

$$
\boldsymbol{L}_{\nu}(x)-I_{-\nu}(x) \approx-\frac{1}{\pi} \sum_{j=0}^{\infty} \frac{(-1)^{j+1} \Gamma(j+1 / 2)}{\Gamma(\nu+1 / 2-j)(x / 2)^{2 j-\nu+1}}
$$

which to leading order is

$$
\boldsymbol{L}_{\nu}(x)-I_{-\nu}(x) \approx-\frac{1}{\sqrt{\pi} \Gamma(\nu+1 / 2)}\left(\frac{2}{x}\right)^{1-\nu}
$$

gives us

$$
\begin{aligned}
\left\langle 0_{\text {in }}\left|\partial_{t} \phi\left(\overrightarrow{x_{1}}, t\right) \partial_{t} \phi\left(\overrightarrow{x_{2}}, t\right)\right| 0_{\text {in }}\right\rangle_{t d} & =-\int_{0}^{\pi} d \phi \frac{m\left(r^{2} \sin ^{2}(\phi)+4 t^{2}\right)}{8 \pi^{2}\left(r^{2} \sin ^{2}(\phi)-4 t^{2}\right)^{2}} \\
& =-\frac{m t}{4 \pi\left(4 t^{2}-r^{2}\right)^{3 / 2}}=-\frac{m}{32 \pi t^{2}}+\mathcal{O}\left(t^{-4}\right)
\end{aligned}
$$

\section{C.2 3+1 dimensions}

The details of calculation of correlators in $d=3$ are presented here.

\section{C.2.1 CC correlator}

The integral in equation (2.27) for only $\kappa_{2}$ non-zero, can be evaluated directly in Mathematica. Even so we calculate it by hand as we would eventually need to do so in the $\mathrm{gCC}_{4}$ case. Performing the angular integral we land up with

$$
\begin{aligned}
\left\langle f_{2}\left|\phi\left(x_{1}, t\right) \phi\left(x_{2}, t\right)\right| f_{2}\right\rangle & =\frac{1}{4 \pi^{2} r} \int_{0}^{\infty} d k \sin (k r)\left[\operatorname{coth}\left(2 \kappa_{2} k\right)-\operatorname{csch}\left(2 \kappa_{2} k\right) \cos (2 k t)\right] \\
& =\frac{1}{8 \pi^{2} i r} \int_{-\infty}^{\infty} d k e^{i k r}\left[\operatorname{coth}\left(2 \kappa_{2} k\right)-\operatorname{csch}\left(2 \kappa_{2} k\right) \cos (2 k t)\right] \\
& =\frac{1}{8 \pi^{2} i r} \int_{-\infty}^{\infty} d k[\underbrace{\frac{\cosh \left(2 \kappa_{2} k\right) e^{i k r}}{\sinh \left(2 \kappa_{2} k\right)}}_{\mathrm{A}}-\underbrace{\frac{e^{i k\left(r+t_{+}\right)}}{2 \sinh \left(2 \kappa_{2} k\right)}}_{\mathrm{B}}-\underbrace{\frac{e^{i k\left(r-t_{+}\right)}}{2 \sinh \left(2 \kappa_{2} k\right)}}_{\mathrm{C}}]
\end{aligned}
$$




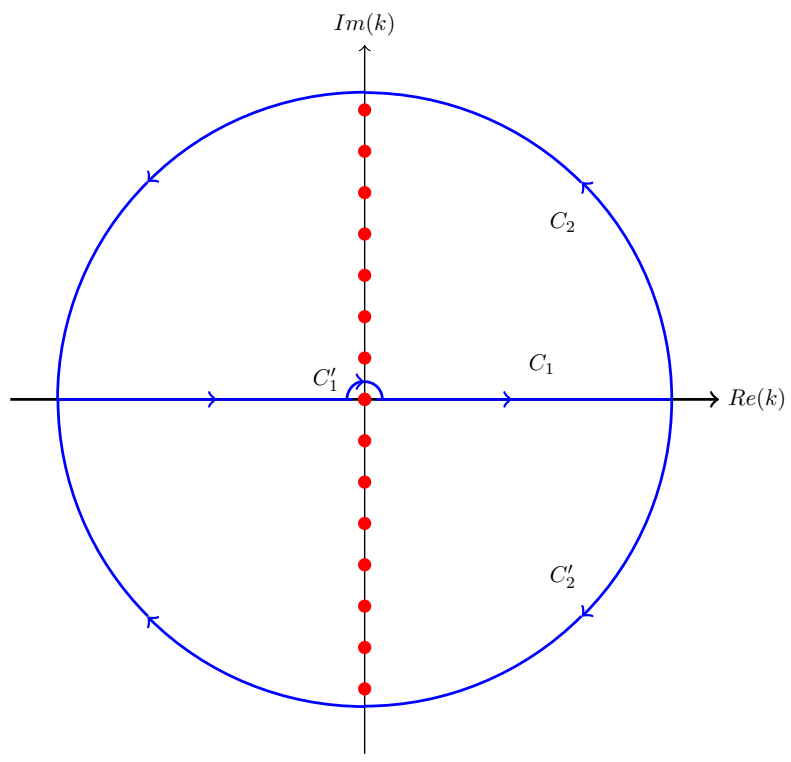

Figure 6. Singularity Structure of Integrand in eq. (2.27).

where we have used $t_{+}=2 t$. Now $\sinh \left(2 \kappa_{2} k\right)$ has simple poles at $2 \kappa_{2} k=i n \pi$ or $k=i n \pi / 2 \kappa 2$ for any integer $\mathrm{n}$. We are interested in the $t_{+}>r$ limit, so we close the contour in the upper half plane for terms $\mathrm{A}$ and $\mathrm{B}$ while in the lower half for $\mathrm{C}$. The residue at these poles is easily calculated

$$
\begin{aligned}
\operatorname{Res}_{A}(i n \pi / 2 \kappa 2) & =\frac{1}{2 \kappa_{2}} e^{-n \pi r / 2 \kappa_{2}} \\
\operatorname{Res}_{B}(i n \pi / 2 \kappa 2) & =\frac{(-1)^{n}}{4 \kappa_{2}} e^{-n \pi\left(t_{+}+r\right) / 2 \kappa_{2}} \\
\operatorname{Res}_{C}(-i n \pi / 2 \kappa 2) & =\frac{(-1)^{n}}{4 \kappa_{2}} e^{-n \pi\left(t_{+}-r\right) / 2 \kappa_{2}}
\end{aligned}
$$

We take half the contribution from the $k=0$ pole. Thus we can write the integral as

$$
\begin{aligned}
& \left\langle f_{2}\left|\phi\left(x_{1}, t\right) \phi\left(x_{2}, t\right)\right| f_{2}\right\rangle \\
& =\frac{2 \pi i}{8 \pi^{2} i r}\left[\frac{1}{2 \kappa_{2}}\left(\frac{1}{2}-\frac{1}{4}+\frac{1}{4}\right)+\sum_{n=1}^{\infty}\left(\frac{e^{-n \pi r / 2 \kappa_{2}}}{2 \kappa_{2}}-\frac{(-1)^{n} e^{-n \pi\left(t_{+}+r\right) / 2 \kappa_{2}}}{4 \kappa_{2}}+\frac{(-1)^{n} e^{-n \pi\left(t_{+}-r\right) / 2 \kappa_{2}}}{4 \kappa_{2}}\right)\right] \\
& =\frac{2 \pi i}{16 \pi^{2} i r \kappa_{2}}\left[\sum_{n=1}^{\infty} e^{-n \pi r / 2 \kappa_{2}}+\frac{1}{2}-\frac{1}{2}\left(\sum_{n=1}^{\infty}(-1)^{n} e^{-n \pi\left(t_{+}+r\right) / 2 \kappa_{2}}+\frac{1}{2}\right)+\frac{1}{2}\left(\sum_{n=1}^{\infty}(-1)^{n} e^{-n \pi\left(t_{+}-r\right) / 2 \kappa_{2}}+\frac{1}{2}\right)\right] \\
& \left\langle f_{2}\left|\phi\left(x_{1}, t\right) \phi\left(x_{2}, t\right)\right| f_{2}\right\rangle \\
& =\frac{1}{32 \pi \kappa_{2} r}\left[2 \operatorname{coth}\left(\frac{\pi r}{4 \kappa_{2}}\right)+\tanh \left(\frac{\pi(2 t-r)}{4 \kappa_{2}}\right)-\tanh \left(\frac{\pi(2 t+r)}{4 \kappa_{2}}\right)\right] \\
& =\frac{1}{32 \pi \kappa_{2} r}\left[2 \operatorname{coth}\left(\frac{\pi r}{4 \kappa_{2}}\right)-\tanh \left(\frac{\pi r}{2 \kappa_{2}}\right)\left\{1-\tanh \left(\frac{\pi(2 t-r)}{4 \kappa_{2}}\right) \tanh \left(\frac{\pi(2 t+r)}{4 \kappa_{2}}\right)\right\}\right]
\end{aligned}
$$


we have already done the summations

$$
\begin{gathered}
\sum_{n=1}^{\infty} e^{-n s}+\frac{1}{2}=\frac{1}{e^{s}-1}+\frac{1}{2}=\frac{1}{2} \operatorname{coth}(s / 2) \\
\sum_{n=1}^{\infty}(-1)^{n} e^{-n s}+\frac{1}{2}=-\frac{1}{e^{s}+1}+\frac{1}{2}=\frac{1}{2} \tanh (s / 2)
\end{gathered}
$$

The $\frac{1}{2}$ 's are coming from the $n=0$ pole at the origin. The slowest decaying transient is $\left(t \gg \kappa_{2}>r\right)$

$$
-\frac{1}{16 \kappa_{2}^{2}} e^{-\pi t / \kappa_{2}}
$$

This is interpreted as the contribution of the pole nearest to the origin (but not the origin itself). The transients from other poles decay much faster than this. Also note that the answer for $r>2 t$, for which all contours are closed in the UHP, comes out to be the same as $r<2 t$.

\section{C.2.2 $\mathrm{gCC}_{4}$ correlator}

The singularity structure of the correlator in the $\mathrm{gCC}_{4}$ state $(2.24)$ is similar to CC state.

$$
\begin{aligned}
& \left\langle f_{4}\left|\phi\left(\overrightarrow{0}, t_{1}\right) \phi\left(\vec{r}, t_{2}\right)\right| f_{4}\right\rangle \\
& =\int \frac{d^{3} k}{(2 \pi)^{3}} \frac{e^{\iota \vec{k} \cdot \vec{r}}}{2|\vec{k}|}\left[\operatorname{coth}\left(2 \kappa_{2}|\vec{k}|+2 \kappa_{4}|\vec{k}|^{3}\right)-\cos (2|\vec{k}| t) \operatorname{csch}\left(2 \kappa_{2}|\vec{k}|+\kappa_{4}|\vec{k}|^{3}\right)\right] \\
& =\frac{1}{8 \pi^{2} \iota r} \int_{\infty}^{\infty} d k[\underbrace{\frac{e^{\iota k r} \cosh \left(2 \kappa_{2}|\vec{k}|+\kappa_{4}|\vec{k}|^{3}\right)}{\sinh \left(2 \kappa_{2}|\vec{k}|+\kappa_{4}|\vec{k}|^{3}\right)}}_{\mathrm{A}}-\underbrace{\frac{e^{\iota k(r+2 t)}}{2 \sinh \left(2 \kappa_{2}|\vec{k}|+\kappa_{4}|\vec{k}|^{3}\right)}}_{B}-\underbrace{\frac{e^{\iota k(r-2 t)}}{2 \sinh \left(2 \kappa_{2}|\vec{k}|+\kappa_{4}|\vec{k}|^{3}\right)}}_{C}]
\end{aligned}
$$

Doing this integral exactly is hard. But it is simpler, and more instructive, to figure out the behaviour of the integral by looking at the poles of the integrand in the complex $k$-plane. These are situated at the roots of $2 \kappa_{2} k+2 \kappa_{4} k^{3}=i n \pi$. Introducing a dimensionless parameter $\bar{\kappa}_{4}=\kappa_{4} / \kappa_{2}^{3}$, in the small $\bar{\kappa}_{4}$ expansion, we find that roots of the above equation are

$$
\begin{aligned}
& k_{1}(n)=\frac{i \pi}{2 \kappa_{2}}\left(n+\frac{\pi^{2} n^{3}}{4} \overline{\kappa_{4}}+O\left({\overline{\kappa_{4}}}^{2}\right)\right) \\
& k_{2}(n)=\frac{i}{\kappa_{2}}\left(\frac{1}{\sqrt{\overline{\kappa_{4}}}}-\frac{\pi n}{4}-\frac{3 \pi^{2} n^{2}}{32} \sqrt{\overline{\kappa_{4}}}+O\left(\overline{\kappa_{4}}\right)\right) \\
& k_{3}(n)=-\frac{i}{\kappa_{2}}\left(\frac{1}{\sqrt{\overline{\kappa_{4}}}}+\frac{\pi n}{4}-\frac{3 \pi^{2} n^{2}}{32} \sqrt{\kappa_{4}}+O\left(\overline{\kappa_{4}}\right)\right)
\end{aligned}
$$

As we saw earlier (in particular, in the previous subsection), the leading large time behaviour is given by the slowest transient. So we only need to calculate the contribution of the poles nearest to the origin i.e. from $k_{1}(n)$ with $n= \pm 1$. Both $k_{2}(n)$ and $k_{3}(n)$ are very large due to the small $\sqrt{\overline{\kappa_{4}}}$ in the denominator. 
Thus forgetting about all the other poles we do the integral. The residues are

$$
\begin{aligned}
\operatorname{Res}_{A}\left(k_{1}(1)\right) & =\frac{1}{2 \kappa_{2}}\left(1+\frac{3}{4 \pi^{2}}{\overline{\kappa_{4}}}+\mathcal{O}\left({\overline{\kappa_{4}}}^{2}\right)\right) \exp \left[-\frac{\pi r}{2 \kappa_{2}}\left(1+\frac{\pi^{2}}{4}{\overline{\kappa_{4}}}^{2} \mathcal{O}\left({\overline{\kappa_{4}}}^{2}\right)\right)\right] \\
\operatorname{Res}_{B}\left(k_{1}(1)\right) & =-\frac{1}{4 \kappa_{2}}\left(1+\frac{3}{4 \pi^{2}} \bar{\kappa}_{4}+\mathcal{O}\left({\overline{\kappa_{4}}}^{2}\right)\right) \exp \left[-\frac{\pi(2 t+r)}{2 \kappa_{2}}\left(1+\frac{\pi^{2}}{4}{\overline{\kappa_{4}}}^{2}+\mathcal{O}\left({\overline{\kappa_{4}}}^{2}\right)\right)\right] \\
\operatorname{Res}_{C}\left(k_{1}(-1)\right) & =-\frac{1}{4 \kappa_{2}}\left(1+\frac{3}{4 \pi^{2}} \bar{\kappa}_{4}+\mathcal{O}\left({\overline{\kappa_{4}}}^{2}\right)\right) \exp \left[-\frac{\pi(2 t-r)}{2 \kappa_{2}}\left(1+\frac{\pi^{2}}{4}{\overline{\kappa_{4}}}^{2} \mathcal{O}\left({\overline{\kappa_{4}}}^{2}\right)\right)\right] \\
\operatorname{Res}_{A}\left(k_{1}(0)\right) & =\frac{1}{2 \kappa_{2}} ; \quad \operatorname{Res}_{B}\left(k_{1}(0)\right)=\frac{1}{4 \kappa_{2}} ; \quad \operatorname{Res}_{C}\left(k_{1}(0)\right)=\frac{1}{4 \kappa_{2}}
\end{aligned}
$$

As before we close the contour in the upper half plane for terms A and B while in the lower half for $\mathrm{C}$. The result is

$$
\begin{aligned}
& \left\langle f_{4}\left|\phi\left(\overrightarrow{0}, t_{1},\right) \phi\left(\vec{r}, t_{2}\right)\right| f_{4}\right\rangle \\
& =\frac{1}{4 \pi r \kappa_{2}}\left(\operatorname{Res}_{A}\left(k_{1}(1)\right)+\frac{1}{2} \operatorname{Res}_{A}\left(k_{1}(0)\right)-\operatorname{Res}_{B}\left(k_{1}(1)\right)\right. \\
& \left.-\frac{1}{2} \operatorname{Res}_{B}\left(k_{1}(0)\right)+\operatorname{Res}_{C}\left(k_{1}(1)\right)+\frac{1}{2} \operatorname{Res}_{C}\left(k_{1}(0)\right)\right) \\
& =\frac{1}{16 \pi r \kappa_{2}}+\frac{1}{16 \pi r \kappa_{2}}\left(1+\frac{3 \pi^{2}}{4} \bar{\kappa}_{4}+\mathcal{O}\left({\overline{\kappa_{4}}}^{2}\right)\right)\left\{2 \exp \left[-\frac{\pi}{2 \kappa_{2}}\left(1+\frac{\pi^{2}}{4}{\overline{\kappa_{4}}}^{2} \mathcal{O}\left({\overline{\kappa_{4}}}^{2}\right)\right) r\right]\right. \\
& \left.+\exp \left[-\frac{\pi}{2 \kappa_{2}}\left(1+\frac{\pi^{2}}{4} \bar{\kappa}_{4}+\mathcal{O}\left({\overline{\kappa_{4}}}^{2}\right)\right)(2 t+r)\right]-\exp \left[-\frac{\pi}{2 \kappa_{2}}\left(1+\frac{\pi^{2}}{4}{\overline{\kappa_{4}}}^{2}+\mathcal{O}\left(\bar{\kappa}_{4}^{2}\right)\right)(2 t-r)\right]\right\} \\
& +\mathcal{O}\left(\exp \left(-2 \pi t / \kappa_{2}\right)\right)
\end{aligned}
$$

The exact value of $k_{1}(1)$ is $k_{1}(1)=i \gamma$ where

$$
\gamma=\frac{-26^{2 / 3}+\sqrt[3]{6}\left(\sqrt{48-81 \pi^{2} \bar{\kappa}_{4}}+9 i \pi \sqrt{\bar{\kappa}_{4}}\right)^{2 / 3}}{6 \kappa_{2} \sqrt{\bar{\kappa}_{4}} \sqrt[3]{\sqrt{48-81 \pi^{2} \bar{\kappa}_{4}}+9 i \pi \sqrt{\bar{\kappa}_{4}}}}
$$

which implies that the exponentials appearing above are of the form

$$
e^{-\gamma r}, e^{-\gamma(2 t \pm r)}
$$

\section{C.2.3 Ground state correlator}

The above method of estimating the time-dependence at large times can also be applied to the ground state as it is just a particular squeezed state. But here we will use the special relation between correlators in $1+1$ and $3+1$ given by the action of the operation $\frac{-1}{2 \pi r} \partial_{r}$ as already mentioned before in section 3.1. Lets see this in detail. The most general gCC correlator in $1+1$ is given by

$$
\left\langle f\left|\phi\left(x_{1}, t_{1}\right) \phi\left(x_{2}, t_{2}\right)\right| f\right\rangle=\int \frac{d k}{(2 \pi)} \frac{e^{i k r}}{2 k} \operatorname{csch}(2 \kappa(k))\left[\cos \left(2 i \kappa(k)+k t_{-}\right)-\cos \left(k t_{+}\right)\right]
$$


where $r=\left(x_{1}-x_{2}\right)$. Acting with $\frac{-1}{2 \pi r} \partial_{r}$ on this expression we obtain

$$
=\frac{1}{8 \pi^{2} i r} \int_{-\infty}^{\infty} d k e^{i k r} \operatorname{csch}(2 \kappa(k))\left[\cos \left(2 i \kappa(k)+k t_{-}\right)-\cos \left(k t_{+}\right)\right]
$$

This is precisely the expression for the most general gCC correlator in $3+1$ after having done the angular integral. We are going to use this operation on the $1+1$ ground state correlator to obtain the ground state correlator in $3+1$. In $1+1$ we are able to do the integral exactly in terms of Meijer G-functions.

$$
\begin{aligned}
\left\langle 0_{\text {in }}\left|\phi\left(x_{1}, t\right) \phi\left(x_{2}, t\right)\right| 0_{\text {in }}\right\rangle= & -\frac{1}{16 \pi}\left\{-2 G_{1,3}^{2,1}\left(\frac{m^{2} r^{2}}{4} \mid \begin{array}{c}
\frac{3}{2} \\
0,1, \frac{1}{2}
\end{array}\right)+G_{1,3}^{2,1}\left(\frac{1}{4} m^{2}(r+2 t)^{2} \mid \begin{array}{c}
\frac{3}{2} \\
0,1, \frac{1}{2}
\end{array}\right)\right. \\
& \left.+G_{1,3}^{2,1}\left(\frac{1}{4} m^{2}(r-2 t)^{2} \mid \begin{array}{c}
\frac{3}{2} \\
0,1, \frac{1}{2}
\end{array}\right)-8 K_{0}(m r)\right\}
\end{aligned}
$$

Acting with $\frac{-1}{2 \pi r} \partial_{r}$ on this we obtain the correlator in $3+1$.

$$
\begin{aligned}
\left\langle 0_{\text {in }}\left|\phi\left(x_{1}, t_{1}\right) \phi\left(x_{2}, t_{2}\right)\right| 0_{\text {in }}\right\rangle= & \frac{1}{32 \pi^{2} r^{2}\left(r^{2}-4 t^{2}\right)}\left\{-2\left(r^{2}-4 t^{2}\right) G_{1,3}^{2,1}\left(\frac{m^{2} r^{2}}{4} \mid \begin{array}{c|c}
\frac{3}{2} \\
0,1, \frac{1}{2}
\end{array}\right)\right. \\
& +r(r+2 t) G_{1,3}^{2,1}\left(\frac{1}{4} m^{2}(r-2 t)^{2} \mid \begin{array}{c}
\frac{3}{2} \\
0,1, \frac{1}{2}
\end{array}\right) \\
& +r(r-2 t)\left[G_{1,3}^{2,1}\left(\frac{1}{4} m^{2}(r+2 t)^{2} \mid \begin{array}{c}
\frac{3}{2} \\
0,1, \frac{1}{2}
\end{array}\right)\right. \\
& \left.\left.+2 m(r+2 t)\left(K_{1}(m(r+2 t))-K_{1}(2 m t-m r)+2 K_{1}(m r)\right)\right]\right\}
\end{aligned}
$$

\section{C.2.4 The thermal correlator}

The 2-point function in the thermal ensemble is (D.3)

$$
\begin{aligned}
\langle\phi & \left.\left(\overrightarrow{x_{1}}, t_{1}\right) \phi\left(\overrightarrow{x_{2}}, t_{2}\right)\right\rangle_{\beta} \\
& =\frac{1}{2} \int \frac{d^{3} k}{(2 \pi)^{3}} \frac{e^{\imath \vec{k} \cdot \vec{x}}}{\omega_{\text {out }}}\left[\cos \left(\omega_{\text {out }} t\right) \operatorname{coth}\left(\frac{\mu(k)}{2}\right)-i \sin \left(\omega_{\text {out }} t\right)\right] \\
& =\frac{1}{4 \pi^{2} r} \int_{0}^{\infty} d k \sin (k r)[\cos (k t) \operatorname{coth}(\beta k / 2)-i \sin (k t)] \\
& =\frac{1}{8 \pi^{2} i r} \int_{-\infty}^{\infty} d k e^{i k r}[\cos (k t) \operatorname{coth}(\beta k / 2)-i \sin (k t)] \\
& =\frac{1}{16 \pi^{2} i r} \int_{-\infty}^{\infty} d k\left(e^{i k(r+t)} \operatorname{coth}(\beta k / 2)+e^{i k(r-t)} \operatorname{coth}(\beta k / 2)-e^{i k(r+t)}+e^{i k(r-t)}\right)
\end{aligned}
$$

The last 2 terms give us Dirac-delta functions, while the first 2 terms are easily evaluated using contour integral with simple poles at the location $k=2 i \pi n / \beta$. Since $t>r$, for the 
first term we close the contour in the UHP while for the second term we close in the LHP. The contribution of the pole at the origin cancels. Summing over all the poles we get

$$
\left\langle\phi\left(\overrightarrow{x_{1}}, t_{1}\right) \phi\left(\overrightarrow{x_{2}}, t_{2}\right)\right\rangle_{\beta}=\frac{\operatorname{coth}\left(\frac{\pi(r-t)}{\beta}\right)+\operatorname{coth}\left(\frac{\pi(r+t)}{\beta}\right)}{8 \pi \beta r}+\frac{i \delta(r-t)}{8 \pi r}-\frac{i \delta(r+t)}{8 \pi r}
$$

The thermal auto-correlator is obtained by taking $r \rightarrow 0$

$$
\left\langle\phi\left(\overrightarrow{x_{1}}, t_{1}\right) \phi\left(\overrightarrow{x_{1}}, t_{2}\right)\right\rangle_{\beta}=-\frac{\operatorname{csch}^{2}\left(\frac{\pi t}{\beta}\right)}{4 \beta^{2}}
$$

\section{C.3 4+1 dimensions}

Here the details of calculation of particular correlators in $d=4$ are provided, even though a general method of calculating the time-dependent part is presented in the text for an arbitrary gCC state.

\section{C.3.1 CC correlator}

The time dependent part of eq. (2.27) with only $\kappa_{2}$ non-zero is

$$
\left\langle f_{2}\left|\phi\left(x_{1}, t\right) \phi\left(x_{2}, t\right)\right| f_{2}\right\rangle-\left\langle\phi\left(\overrightarrow{x_{1}}, t\right) \phi\left(\overrightarrow{x_{2}}, t\right)\right\rangle_{\beta}=-\int \frac{d^{d} k}{(2 \pi)^{d}} \frac{e^{i \vec{k} \cdot\left(\overrightarrow{x_{1}}-\overrightarrow{x_{2}}\right)}}{2|\vec{k}|} \operatorname{csch}\left(2 \kappa_{2}|\vec{k}|\right) \cos (2|\vec{k}| t)
$$

which in $4+1$ simplifies to

$$
\begin{aligned}
- & \frac{1}{8 \pi^{2} r} \int_{0}^{\infty} d k k J_{1}(k r) \operatorname{csch}\left(2 \kappa_{2} k\right) \cos (2 k t) \\
= & -\frac{1}{8 \pi^{3} r} \int_{0}^{\infty} d k k \int_{0}^{\pi} d \phi \cos (k r \sin \phi-\phi) \operatorname{csch}\left(2 \kappa_{2} k\right) \cos (2 k t) \\
= & -\frac{1}{16 \pi^{3} r} \int_{0}^{\pi} d \phi \int_{0}^{\infty} d k k \operatorname{csch}\left(2 \kappa_{2} k\right)(\cos (k(2 t+r \sin \phi)-\phi)+\cos (k(2 t-r \sin \phi)+\phi)) \\
= & -\frac{1}{16 \pi^{3} r} \int_{0}^{\pi} d \phi \int_{0}^{\infty} d k k \operatorname{csch}\left(2 \kappa_{2} k\right)[\cos (k(2 t+r \sin \phi)-\phi)+\cos (k(2 t-r \sin \phi)+\phi)] \\
= & -\frac{1}{256 \pi^{3} \kappa_{2}^{2} r} \int_{0}^{\pi} d \phi\left[e^{i \phi}\left(\psi^{(1)}\left(\frac{i\left(t_{+}+r \sin \phi\right)}{4 \kappa_{2}}+\frac{1}{2}\right)+\psi^{(1)}\left(\frac{1}{2}-\frac{i\left(t_{+}-r \sin \phi\right)}{4 \kappa_{2}}\right)\right)\right. \\
& \left.+e^{-i \phi}\left(\psi^{(1)}\left(\frac{i\left(t_{+}-r \sin \phi\right)}{4 \kappa_{2}}+\frac{1}{2}\right)+\psi^{(1)}\left(\frac{1}{2}-\frac{i\left(t_{+}+r \sin \phi\right)}{4 \kappa_{2}}\right)\right)\right] \\
\approx & \int d \phi\left[\frac{\sin ^{2}(\phi)}{\left(\left(16 \pi^{3}\right) \kappa_{2}\right) t_{+}^{2}}+\frac{\sin ^{2}(\phi)\left(8 \kappa_{2}^{2}+r^{2}(-\cos (2 \phi))+r^{2}\right)}{\left(\left(32 \pi^{3}\right) \kappa_{2}\right) t_{+}^{4}}+O\left(\frac{1}{t^{6}}\right)\right] \\
= & \frac{1}{128 \pi^{2} \kappa_{2} t^{2}}+\frac{3 r^{2}+16 \kappa_{2}^{2}}{2^{11} \pi^{2} \kappa_{2} t^{4}}+O\left(t^{-6}\right)
\end{aligned}
$$

In the second line we used the integral representation $J_{n}(x)=\frac{1}{\pi} \int_{0}^{\pi} d \tau \cos (x \sin \tau-n \tau)$ of the Bessel function. Then we perform the $k$ integral to get Polygamma functions. In the end we do the $\phi$ integral after series expanding Polygammas around $t=\infty$ to get leading large $t$ answer. 


\section{C.3.2 Ground state correlator}

The time-dependent part of the equal-time ground state correlator here is

$$
\begin{aligned}
= & -\frac{m^{2}}{16 \pi^{2} r} \int_{0}^{\infty} \frac{d k}{\sqrt{k^{2}+m^{2}}} J_{1}(k r) \cos \left(k t_{+}\right) \\
= & -\frac{m^{2}}{16 \pi^{3} r} \int_{0}^{\infty} \frac{d k}{\sqrt{k^{2}+m^{2}}} \int_{0}^{\pi} d \phi \cos (k r \sin \phi-\phi) \cos \left(k t_{+}\right) \\
= & -\frac{m^{2}}{32 \pi^{3} r} \int_{0}^{\infty} \frac{d k}{\sqrt{k^{2}+m^{2}}} \int_{0}^{\pi} d \phi\left[\cos \left(k a_{+}-\phi\right)+\cos \left(k a_{-}+\phi\right)\right] \\
= & -\frac{m^{2}}{64 \pi^{3} r} \int_{0}^{\infty} \frac{d k}{\sqrt{k^{2}+m^{2}}} \int_{0}^{\pi} d \phi\left[e^{i\left(k a_{+}-\phi\right)}+e^{-i\left(k a_{+}-\phi\right)}+e^{i\left(k a_{-}+\phi\right)}+e^{-i\left(k a_{-}+\phi\right)}\right] \\
= & -\frac{m^{2}}{64 \pi^{3} r} \int_{0}^{\infty} \frac{d k}{\sqrt{k^{2}+m^{2}}} \int_{0}^{\pi} d \phi\left[e^{-i \phi}\left(e^{i k a_{+}}+e^{-i k a_{-}}\right)+e^{i \phi}\left(e^{-i k a_{+}}+e^{i k a_{-}}\right)\right] \\
= & -\frac{m^{2}}{64 \pi^{3} r} \int_{0}^{\pi} d \phi\left[\pi \operatorname { s i n } \phi \left(-\boldsymbol{L}_{0}\left(m\left(t_{+}+r \sin \phi\right)\right)+\boldsymbol{L}_{0}\left(m\left(t_{+}-r \sin \phi\right)\right)\right.\right. \\
& \left.+I_{0}\left(m\left(t_{+}+r \sin \phi\right)\right)-I_{0}\left(m\left(t_{+}-r \sin \phi\right)\right)\right) \\
& \left.+2 \cos (\phi)\left(K_{0}\left(m\left(t_{+}+r \sin \phi\right)\right)+K_{0}\left(m\left(t_{+}-r \sin \phi\right)\right)\right)\right]
\end{aligned}
$$

In the second line we have used the integral representation for $J_{1}(k r)$. Then after some manipulation we are able to integrate over momentum $k . \boldsymbol{L}_{0}(x)$ is the Modified Struve function and $I_{0}(x)$ is the Bessel-I function. Now using the asymptotic form of these functions, eq. (C.5)

$$
\boldsymbol{L}_{\nu}(x)-I_{-\nu}(x) \approx-\frac{1}{\sqrt{\pi} \Gamma(\nu+1 / 2)}\left(\frac{2}{x}\right)^{1-\nu}
$$

and knowing that $K_{0}(x) \approx e^{-x} / \sqrt{x}$ is exponentially suppressed at large $x$, one gets

$$
\int_{0}^{\pi} d \phi \frac{m \sin ^{2} \phi}{64 \pi^{3} t^{2}}=\frac{m}{128 \pi^{2} t^{2}}
$$

\section{C.3.3 The thermal correlator}

The 2-point function in the thermal ensemble is (D.3)

$$
\begin{aligned}
\left\langle\phi\left(\overrightarrow{x_{1}}, t_{1}\right) \phi\left(\overrightarrow{x_{2}}, t_{2}\right)\right\rangle_{\beta} & =\frac{1}{2} \int \frac{d^{4} k}{(2 \pi)^{4}} \frac{e^{\imath \vec{k} \cdot \vec{x}}}{\omega_{\text {out }}}\left[\cos \left(\omega_{\text {out }} t\right) \operatorname{coth}\left(\frac{\mu(k)}{2}\right)-i \sin \left(\omega_{\text {out }} t\right)\right] \\
& =\frac{1}{8 \pi^{2} r} \int_{0}^{\infty} d k J_{1}(k r) k[\cos (k t) \operatorname{coth}(\beta k / 2)-i \sin (k t)] \\
& =\frac{1}{8 \pi^{2} r} \int_{0}^{\infty} d k J_{1}(k r) k\left[\frac{2 \cos (k t)}{e^{\beta k}-1}+e^{-i k t}\right]
\end{aligned}
$$

We have added and subtracted 1 from the coth to get to the final line. Now to calculate the first term we use the same reasoning as in section 3.2.1. To this end we define dimensionless 
momentum $p=k t$ and expand the integrand at large $t$ (see eq. (3.17))

$$
\begin{aligned}
\frac{1}{8 \pi^{2} r} \int_{0}^{\infty} d k J_{1}(k r) k \frac{2 \cos (k t)}{e^{\beta k}-1} & =\frac{1}{4 \pi^{2} r t^{2}} \int_{0}^{\infty} d p J_{1}(p r / t) p \frac{\cos (p)}{e^{\beta p / t}-1} \\
& =\frac{1}{16 \pi^{2} t^{2} \beta}\left[I(1)-\frac{\beta}{2 t} I(2)+\frac{-3 r^{2}+2 \beta^{2}}{24 t^{2}} I(3)+\mathcal{O}\left(t^{-3}\right)\right] \\
& =-\frac{1}{8 \pi^{2} \beta t^{2}}+\frac{-3 r^{2}+2 \beta^{2}}{32 \pi^{2} \beta t^{4}}+\mathcal{O}\left(t^{-6}\right)
\end{aligned}
$$

We have already rotated the contours appropriately and used the notation $I(n)$ of eq. (3.18). The $e^{-i k t}$ term is easily evaluated to give

$$
\frac{1}{8 \pi^{2} r} \int_{0}^{\infty} d k J_{1}(k r) k e^{-i k t}=\frac{i}{8 \pi^{2} t^{3}\left(1-\frac{r^{2}}{t^{2}}\right)^{3 / 2}}
$$

This is just the UV singularity one expects in $d=4$. Combining we have

$$
\left\langle\phi\left(\overrightarrow{x_{1}}, t_{1}\right) \phi\left(\overrightarrow{x_{2}}, t_{2}\right)\right\rangle_{\beta}=\frac{i}{8 \pi^{2} t^{3}\left(1-\frac{r^{2}}{t^{2}}\right)^{3 / 2}}-\frac{1}{8 \pi^{2} \beta t^{2}}+\frac{-3 r^{2}+2 \beta^{2}}{32 \pi^{2} \beta t^{4}}+\mathcal{O}\left(t^{-6}\right)
$$

The thermal auto-correlator is obtained by taking $r \rightarrow 0$

$$
\left\langle\phi\left(\overrightarrow{x_{1}}, t_{1}\right) \phi\left(\overrightarrow{x_{2}}, t_{2}\right)\right\rangle_{\beta}=\frac{i}{8 \pi^{2} t^{3}}-\frac{1}{8 \pi^{2} \beta t^{2}}+\frac{\beta}{16 \pi^{2} t^{4}}+\mathcal{O}\left(t^{-6}\right)
$$

\section{GGE correlator}

For the GGE "density matrix" $\rho=\frac{1}{Z} \exp \left(-\sum_{k} \mu(k) \hat{N}(k)\right)$, its easy to calculate the partition function

$$
\begin{aligned}
Z & =\operatorname{tr} \exp \left(-\sum_{k} \mu(k) \hat{N}(k)\right) \\
& =\sum_{\left\{N_{k}\right\}}\left\langle\left\{N_{k}\right\}\left|e^{\left.-\sum_{k} \mu(k) \hat{N}(k)\right)}\right|\left\{N_{k}\right\}\right\rangle=\sum_{\left\{N_{k}\right\}}\left\langle\left\{N_{k}\right\}\left|\prod_{k} e^{-\mu(k) \hat{N}(k))}\right|\left\{N_{k}\right\}\right\rangle \\
& =\prod_{k} \sum_{N_{k}}\left\langle N_{k}\left|e^{-\mu(k) \hat{N}(k))}\right| N_{k}\right\rangle=\prod_{k} \sum_{N_{k}=1}^{\infty} e^{-\mu(k) \hat{N}_{k}} \\
Z & =\prod_{k}\left(1-e^{-\mu(k)}\right)^{-1}
\end{aligned}
$$

Starting with the GGE 2-point function

$$
\begin{aligned}
\left\langle\phi\left(\overrightarrow{x_{1}}, t_{1}\right) \phi\left(\overrightarrow{x_{2}}, t_{2}\right)\right\rangle_{\rho} & =\frac{1}{Z} \operatorname{tr}\left(e^{-\sum_{\vec{k}} \mu(\vec{k}) \hat{N}(\vec{k})} \phi\left(\overrightarrow{x_{1}}, t_{1}\right) \phi\left(\overrightarrow{x_{2}}, t_{2}\right)\right) \\
& =\frac{1}{Z} \sum_{\left\{N_{\vec{k}}\right\}}\left\langle\left\{N_{\vec{k}}\right\}\left|e^{-\sum_{\vec{k}} \mu(\vec{k}) \hat{N}(\vec{k})} \phi\left(\overrightarrow{x_{1}}, t_{1}\right) \phi\left(\overrightarrow{x_{2}}, t_{2}\right)\right|\left\{N_{\vec{k}}\right\}\right\rangle
\end{aligned}
$$


In the second line we have used the occupation number basis. Using the partial Fourier transform for the field and the mode expansion

$$
\phi(\vec{x}, t)=\int e^{i \vec{k} \cdot \vec{x}} \phi(\vec{k}, t) \frac{d^{d} k}{(2 \pi)^{d}}
$$

where $\phi(\vec{k}, t)=a(\vec{k}) u(\vec{k}, t)+a^{\dagger}(\overrightarrow{-k}) u^{*}(\overrightarrow{-k}, t)$, gives

$$
\begin{aligned}
& \left\langle\phi\left(\overrightarrow{x_{1}}, t_{1}\right) \phi\left(\overrightarrow{x_{2}}, t_{2}\right)\right\rangle_{\rho} \\
& =\frac{1}{Z} \sum_{\left\{N_{\vec{k}}\right\}}\left\langle\left\{N_{\vec{k}}\right\}\right| e^{-\sum_{\vec{k}} \mu(\vec{k}) \hat{N}(\vec{k})} \iint \frac{d^{d} k}{(2 \pi)^{d}} \frac{d^{d} q}{(2 \pi)^{d}} e^{\iota\left(\vec{k} \cdot \overrightarrow{x_{1}}+\vec{q} \cdot \overrightarrow{x_{2}}\right)} \\
& \quad\left(a(\vec{k}) u\left(\vec{k}, t_{1}\right)+a^{\dagger}(\overrightarrow{-k}) u^{*}\left(\overrightarrow{-k}, t_{1}\right)\right)\left(a(\vec{q}) u\left(\vec{q}, t_{2}\right)+a^{\dagger}(\overrightarrow{-q}) u^{*}\left(\overrightarrow{-q}, t_{2}\right)\right)\left|\left\{N_{\vec{k}}\right\}\right\rangle
\end{aligned}
$$

Out of the resulting four terms only two terms give non-zero values.

$$
\begin{aligned}
& \left\langle\phi\left(\overrightarrow{x_{1}}, t_{1}\right) \phi\left(\overrightarrow{x_{2}}, t_{2}\right)\right\rangle_{\rho} \\
& =\frac{1}{Z} \iint \frac{d^{d} k}{(2 \pi)^{d}} \frac{d^{d} q}{(2 \pi)^{d}} e^{\iota\left(\vec{k} \cdot \overrightarrow{x_{1}}+\vec{q} \cdot \overrightarrow{x_{2}}\right)} \sum_{\left\{N_{\vec{k}}\right\}} e^{-\sum_{\vec{k}} \mu(\vec{k}) \hat{N}(\vec{k})} \\
& \quad\left\langle\left\{N_{\vec{k}}\right\}\left|\left(a(\vec{k}) u\left(\vec{k}, t_{1}\right) a^{\dagger}(\overrightarrow{-q}) u^{*}\left(\overrightarrow{-q}, t_{2}\right)+a^{\dagger}(\overrightarrow{-k}) u^{*}\left(\overrightarrow{-k}, t_{1}\right) a(\vec{q}) u\left(\vec{q}, t_{2}\right)\right)\right|\left\{N_{\vec{k}}\right\}\right\rangle
\end{aligned}
$$

Using the commutation relation

$$
\left[a(\vec{k}), a^{\dagger}(-\vec{q})\right]=(2 \pi)^{d} \delta^{d}(\vec{k}+\vec{q})
$$

and the form of the number operator

$$
a^{\dagger}(-\vec{q}) a(\vec{k})=N_{\vec{k}}(2 \pi)^{d} \delta^{d}(\vec{k}+\vec{q})
$$

Therefore

$$
\begin{aligned}
& \left\langle\phi\left(\overrightarrow{x_{1}}, t_{1}\right) \phi\left(\overrightarrow{x_{2}}, t_{2}\right)\right\rangle_{\rho} \\
& =\frac{1}{Z} \iint \frac{d^{d} k}{(2 \pi)^{d}} \frac{d^{d} q}{(2 \pi)^{d}} e^{\iota\left(\vec{k} \cdot \overrightarrow{x_{1}}+\vec{q} \cdot \overrightarrow{x_{2}}\right)}(2 \pi)^{d} \delta^{d}(\vec{k}+\vec{q}) \sum_{\left\{N_{\vec{k}}\right\}} e^{-\sum_{\vec{k}} \mu(\vec{k}) \hat{N}(\vec{k})} \\
& \quad \sum_{\left\{N_{\vec{k}}\right\}}\left(\left\langle\left\{N_{\vec{k}}\right\}\left|\left(N_{\vec{k}}+1\right)\right|\left\{N_{\vec{k}}\right\}\right\rangle u\left(\vec{k}, t_{1}\right) u^{*}\left(\overrightarrow{-q}, t_{2}\right)+\left\langle\left\{N_{\vec{k}}\right\}\left|N_{\vec{q}}\right|\left\{N_{\vec{k}}\right\}\right\rangle u^{*}\left(\overrightarrow{-k}, t_{1}\right) u\left(\vec{q}, t_{2}\right)\right)
\end{aligned}
$$

Doing the $\mathrm{q}$ integral for the first term and $\mathrm{k}$ integral for the second and then writing it in terms of a single dummy variable:

$$
\begin{aligned}
& \left\langle\phi\left(\overrightarrow{x_{1}}, t_{1}\right) \phi\left(\overrightarrow{x_{2}}, t_{2}\right)\right\rangle_{\rho} \\
& =\frac{1}{Z} \int \frac{d^{d} k}{(2 \pi)^{d}} \sum_{\left\{N_{\vec{k}}\right\}} e^{-\sum_{\vec{k}} \mu(\vec{k}) \hat{N}(\vec{k})}\left[\left\langle\left\{N_{\vec{k}}\right\}\left|\left(N_{k}+1\right)\right|\left\{N_{\vec{k}}\right\}\right\rangle\right. \\
& \left.\quad u\left(\vec{k}, t_{1}\right) u^{*}\left(\vec{k}, t_{2}\right) e^{\iota \vec{k} \cdot\left(\overrightarrow{x_{1}}-\overrightarrow{x_{2}}\right)}+\left\langle\left\{N_{\vec{k}}\right\}\left|N_{\vec{k}}\right|\left\{N_{\vec{k}}\right\}\right\rangle u^{*}\left(\vec{k}, t_{1}\right) u\left(\vec{k}, t_{2}\right) e^{-\imath \vec{k} \cdot\left(\overrightarrow{x_{1}}-\overrightarrow{x_{2}}\right)}\right]
\end{aligned}
$$


Directly using $\left\langle N_{\vec{k}}\right\rangle=\frac{1}{Z} \sum_{\left\{N_{\vec{k}}\right\}}\left\langle\left\{N_{\vec{k}}\right\}\left|e^{-\sum_{\vec{k}} \mu(\vec{k}) \hat{N}(\vec{k})} N(k)\right|\left\{N_{\vec{k}}\right\}\right\rangle=\bar{N}(k)=\left(e^{\mu(k)}-1\right)^{-1}$, we get

$$
\left\langle\phi\left(\overrightarrow{x_{1}}, t_{1}\right) \phi\left(\overrightarrow{x_{2}}, t_{2}\right)\right\rangle_{\rho}=\frac{1}{2} \int \frac{d^{d} k}{(2 \pi)^{d}} e^{\iota \vec{k} \cdot\left(\overrightarrow{x_{1}}-\overrightarrow{x_{2}}\right)}\left[\frac{u\left(\vec{k}, t_{1}\right) u^{*}\left(\vec{k}, t_{2}\right)}{1-e^{-\mu(k)}}+\frac{u^{*}\left(\vec{k}, t_{1}\right) u\left(\vec{k}, t_{2}\right)}{e^{\mu(k)}-1}\right]
$$

Defining $\vec{x}=\overrightarrow{x_{1}}-\overrightarrow{x_{2}}, t=t_{1}-t_{2}$ and

$$
\begin{aligned}
G_{ \pm} & =\frac{1}{\omega_{\text {out }}\left( \pm e^{ \pm \mu(k)} \mp 1\right)} \\
\left\langle\phi\left(\overrightarrow{x_{1}}, t_{1}\right) \phi\left(\overrightarrow{x_{2}}, t_{2}\right)\right\rangle_{\mu} & =\frac{1}{2} \int \frac{d^{d} k}{(2 \pi)^{d}} e^{l \vec{k} \cdot \vec{x}}\left(G_{-} e^{-\imath \omega_{\text {out }} t}+G_{+} e^{\imath \omega_{\text {out }} t}\right)
\end{aligned}
$$

We can further simplify

$$
\begin{aligned}
& \frac{e^{-\imath \omega_{\text {out }} t}}{\left(1-e^{-\mu(k)}\right)}+\frac{e^{\iota \omega_{\text {out }} t}}{\left(e^{\mu(k)}-1\right)} \\
& \quad=\frac{e^{-\imath \omega_{\text {out }} t} e^{\mu(k) / 2}+e^{\imath \omega_{\text {out }} t} e^{-\mu(k) / 2}}{e^{\mu(k) / 2}-e^{-\mu(k) / 2}} \\
& \quad=\frac{\left(\cos \left(\omega_{\text {out }} t\right)-i \sin \left(\omega_{\text {out }} t\right)\right) e^{\mu(k) / 2}+\left(\cos \left(\omega_{\text {out }} t\right)+i \sin \left(\omega_{\text {out }} t\right)\right) e^{-\mu(k) / 2}}{e^{\mu(k) / 2}-e^{-\mu(k) / 2}} \\
& =\cos \left(\omega_{\text {out }} t\right) \operatorname{coth}\left(\frac{\mu(k)}{2}\right)-i \sin \left(\omega_{\text {out }} t\right)
\end{aligned}
$$

So

$$
\left\langle\phi\left(\overrightarrow{x_{1}}, t_{1}\right) \phi\left(\overrightarrow{x_{2}}, t_{2}\right)\right\rangle_{\mu}=\frac{1}{2} \int \frac{d^{d} k}{(2 \pi)^{d}} \frac{e^{\iota \vec{k} \cdot \vec{x}}}{\omega_{\text {out }}}\left[\cos \left(\omega_{\text {out }} t\right) \operatorname{coth}\left(\frac{\mu(k)}{2}\right)-i \sin \left(\omega_{\text {out }} t\right)\right]
$$

We would be particularly interested in the ETC when $t_{1}=t_{2} \Rightarrow t=0$ then

$$
\left\langle\phi\left(\overrightarrow{x_{1}}, t\right) \phi\left(\overrightarrow{x_{2}}, t\right)\right\rangle_{\mu}=\frac{1}{2} \int \frac{d^{d} k}{(2 \pi)^{d}} \frac{e^{\iota \vec{k} \cdot \vec{x}}}{\omega_{\text {out }}} \operatorname{coth}\left(\frac{\mu(k)}{2}\right)
$$

Open Access. This article is distributed under the terms of the Creative Commons Attribution License (CC-BY 4.0), which permits any use, distribution and reproduction in any medium, provided the original author(s) and source are credited.

\section{References}

[1] M. Rigol, V. Dunjko, V. Yurovsky and M. Olshanii, Relaxation in a completely integrable many-body quantum system: An Ab Initio study of the dynamics of the highly excited states of 1d lattice hard-core bosons, Phys. Rev. Lett. 98 (2007) 050405 [cond-mat/0604476].

[2] M. Rigol, V. Dunjko and M. Olshanii, Thermalization and its mechanism for generic isolated quantum systems, Nature 452 (2008) 854 [arXiv:0708.1324].

[3] P. Calabrese and J. Cardy, Quantum quenches in extended systems, J. Stat. Mech. 2007 (2007) P06008. 
[4] T. Barthel and U. Schollwöck, Dephasing and the Steady State in Quantum Many-Particle Systems, Phys. Rev. Lett. 100 (2008) 100601 [arXiv:0711.4896].

[5] M. Cramer, C.M. Dawson, J. Eisert and T.J. Osborne, Exact Relaxation in a Class of Nonequilibrium Quantum Lattice Systems, Phys. Rev. Lett. 100 (2008) 030602 [cond-mat/0703314] [inSPIRE].

[6] D. Fioretto and G. Mussardo, Quantum Quenches in Integrable Field Theories, New J. Phys. 12 (2010) 055015 [arXiv:0911.3345] [InSPIRE].

[7] A. Iucci and M.A. Cazalilla, Quantum quench dynamics of the Luttinger model, Phys. Rev. A 80 (2009) 063619 [arXiv: 1003.5170].

[8] P. Calabrese, F.H.L. Essler and M. Fagotti, Quantum quench in the transverse field Ising chain: I. Time evolution of order parameter correlators, J. Stat. Mech. 2012 (2012) P07016 [arXiv: 1204.3911].

[9] P. Calabrese, F.H.L. Essler and M. Fagotti, Quantum Quench in the Transverse Field Ising Chain, Phys. Rev. Lett. 106 (2011) 227203 [arXiv:1104.0154] [INSPIRE].

[10] P. Calabrese, F.H.L. Essler and M. Fagotti, Quantum quenches in the transverse field Ising chain: II. Stationary state properties, J. Stat. Mech. 2012 (2012) P07022 [arXiv:1205.2211].

[11] B. Bertini, D. Schuricht and F.H.L. Essler, Quantum quench in the sine-Gordon model, J. Stat. Mech. 1410 (2014) P10035 [arXiv:1405.4813] [INSPIRE].

[12] C. Gogolin and J. Eisert, Equilibration, thermalisation, and the emergence of statistical mechanics in closed quantum systems, Rept. Prog. Phys. 79 (2016) 056001 [arXiv: 1503.07538] [INSPIRE].

[13] F.H.L. Essler, G. Mussardo and M. Panfil, Generalized Gibbs Ensembles for Quantum Field Theories, Phys. Rev. A 91 (2015) 051602 [arXiv:1411.5352] [INSPIRE].

[14] F.H.L. Essler and M. Fagotti, Quench dynamics and relaxation in isolated integrable quantum spin chains, J. Stat. Mech. 2016 (2016) 064002.

[15] L. Vidmar and M. Rigol, Generalized gibbs ensemble in integrable lattice models, J. Stat. Mech. 2016 (2016) 064007.

[16] E. Ilievski, M. Medenjak, T. Prosen and L. Zadnik, Quasilocal charges in integrable lattice systems, J. Stat. Mech. 2016 (2016) 064008.

[17] J.-S. Caux, The quench action, J. Stat. Mech. 2016 (2016) 064006.

[18] S.U.E. Fermi, J.R. Pasta and M. Tsingou, Studies of nonlinear problems, I, Los Alamos Report, LA-1940 (1955).

[19] T. Kinoshita, T. Wenger and D.S. Weiss, A quantum newton's cradle, Nature 440 (2006) 900.

[20] J. Cardy, Thermalization and Revivals after a Quantum Quench in Conformal Field Theory, Phys. Rev. Lett. 112 (2014) 220401 [arXiv: 1403.3040] [INSPIRE].

[21] P. Calabrese and J.L. Cardy, Entanglement entropy and quantum field theory, J. Stat. Mech. 0406 (2004) P06002 [hep-th/0405152] [INSPIRE].

[22] P. Calabrese and J.L. Cardy, Evolution of entanglement entropy in one-dimensional systems, J. Stat. Mech. 0504 (2005) P04010 [cond-mat/0503393] [INSPIRE]. 
[23] P. Calabrese and J.L. Cardy, Time-dependence of correlation functions following a quantum quench, Phys. Rev. Lett. 96 (2006) 136801 [cond-mat/0601225] [INSPIRE].

[24] G. Mandal, R. Sinha and N. Sorokhaibam, Thermalization with chemical potentials, and higher spin black holes, JHEP 08 (2015) 013 [arXiv: 1501.04580] [INSPIRE].

[25] J. Cardy, Quantum Quenches to a Critical Point in One Dimension: some further results, J. Stat. Mech. 1602 (2016) 023103 [arXiv:1507.07266] [INSPIRE].

[26] S. Sotiriadis and P. Calabrese, Validity of the GGE for quantum quenches from interacting to noninteracting models, J. Stat. Mech. 2014 (2014) P07024.

[27] S. Sotiriadis, Memory-preserving equilibration after a quantum quench in a one-dimensional critical model, Phys. Rev. A 94 (2016) 031605 [arXiv:1507.07915] [INSPIRE].

[28] M. Collura, S. Sotiriadis and P. Calabrese, Equilibration of a tonks-girardeau gas following a trap release, Phys. Rev. Lett. 110 (2013) 245301.

[29] M. Collura, M. Kormos and P. Calabrese, Quantum quench in a harmonically trapped one-dimensional bose gas, Phys. Rev. A 97 (2018) 033609.

[30] P. Ruggiero, Y. Brun and J. Dubail, Conformal field theory on top of a breathing one-dimensional gas of hard core bosons, SciPost Phys. 6 (2019) 51.

[31] S.R. Das, D.A. Galante and R.C. Myers, Quantum Quenches in Free Field Theory: Universal Scaling at Any Rate, JHEP 05 (2016) 164 [arXiv: 1602.08547] [INSPIRE].

[32] S.R. Das, S. Hampton and S. Liu, Quantum Quench in Non-relativistic Fermionic Field Theory: Harmonic traps and $2 d$ String Theory, JHEP 08 (2019) 176 [arXiv:1903.07682] [INSPIRE].

[33] S.R. Das, D.A. Galante and R.C. Myers, Universality in fast quantum quenches, JHEP 02 (2015) 167 [arXiv: 1411.7710] [INSPIRE].

[34] S. Sotiriadis, G. Takács and G. Mussardo, Boundary State in an Integrable Quantum Field Theory Out of Equilibrium, Phys. Lett. B 734 (2014) 52 [arXiv:1311.4418] [InSPIRE].

[35] G. Mandal, S. Paranjape and N. Sorokhaibam, Thermalization in 2D critical quench and UV/IR mixing, JHEP 01 (2018) 027 [arXiv:1512.02187] [INSPIRE].

[36] S. Sotiriadis, D. Fioretto and G. Mussardo, Zamolodchikov-Faddeev algebra and quantum quenches in integrable field theories, J. Stat. Mech. 2012 (2012) P02017.

[37] D.X. Horvath, S. Sotiriadis and G. Takács, Initial states in integrable quantum field theory quenches from an integral equation hierarchy, Nucl. Phys. B 902 (2016) 508 [arXiv: 1510.01735] [INSPIRE].

[38] B. Bertini, L. Piroli and P. Calabrese, Quantum quenches in the sinh-gordon model: steady state and one-point correlation functions, J. Stat. Mech. 2016 (2016) 063102.

[39] A.C. Cubero, Planar quantum quenches: computation of exact time-dependent correlation functions at largeN, J. Stat. Mech. 2016 (2016) 083107.

[40] V.E. Korepin, N.M. Bogoliubov and A.G. Izergin, Quantum Inverse Scattering Method and Correlation Functions, Cambridge Monographs on Mathematical Physics, Cambridge University Press (1993).

[41] S. Sotiriadis and J. Cardy, Quantum quench in interacting field theory: A Self-consistent approximation, Phys. Rev. B 81 (2010) 134305 [arXiv: 1002.0167] [INSPIRE]. 
[42] A. Chiocchetta, M. Tavora, A. Gambassi and A. Mitra, Short-time universal scaling and light-cone dynamics after a quench in an isolated quantum system in d spatial dimensions, Phys. Rev. B 94 (2016) 134311 [arXiv:1604.04614] [INSPIRE].

[43] A. Chiocchetta, A. Gambassi, S. Diehl and J. Marino, Dynamical Crossovers in Prethermal Critical States, Phys. Rev. Lett. 118 (2017) 135701 [arXiv:1612.02419] [InSPIRE].

[44] N. Birrell and P. Davies, Quantum Fields in Curved Space, Cambridge Monographs on Mathematical Physics, Cambridge University Press (1984).

[45] S. Bhattacharyya et al., Currents and Radiation from the large D Black Hole Membrane, JHEP 05 (2017) 098 [arXiv: 1611.09310] [INSPIRE].

[46] A. Kaushal and G. Mandal, Approach to thermalization in bosonic $O(N)$ models in arbitrary dimensions, work in progress.

[47] R.H. Jonsson, E. Martin-Martinez and A. Kempf, Information transmission without energy exchange, Phys. Rev. Lett. 114 (2015) 110505 [arXiv: 1405.3988] [InSPIRE].

[48] A. Blasco, L.J. Garay, M. Martin-Benito and E. Martin-Martinez, Violation of the Strong Huygen's Principle and Timelike Signals from the Early Universe, Phys. Rev. Lett. 114 (2015) 141103 [arXiv:1501.01650] [INSPIRE]. 